Supporting Information

\title{
Revealing the Unbinding Kinetics and Mechanism of Type I and Type II Protein Kinase Inhibitors by Local- Scaled Molecular Dynamics Simulations
}

Yu $\mathrm{Du}{ }^{1,2}$, Renxiao Wang $^{1,2,3 *}$

${ }^{1}$ State Key Laboratory of Bioorganic and Natural Products Chemistry, Center for Excellence in Molecular Synthesis, Shanghai Institute of Organic Chemistry, Chinese Academy of Sciences, Shanghai, People's Republic of China;

${ }^{2}$ University of Chinese Academy of Sciences, Beijing, People's Republic of China;

${ }^{3}$ Department of Medicinal Chemistry, School of Pharmacy, Fudan University,

Shanghai, People's Republic of China

* Corresponding author's e-mail: wangrx@ fudan.edu.cn 


\section{TABLE OF CONTENTS}

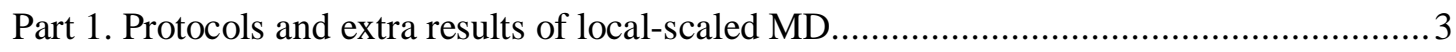

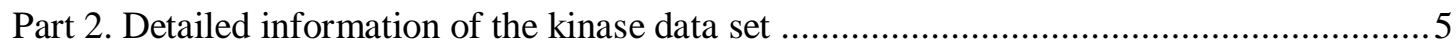

Part 3. Changes of binding energy during local-scaled MD .............................................

Part 4. Local-scaled MD simulation with a finer scaling protocol........................................26

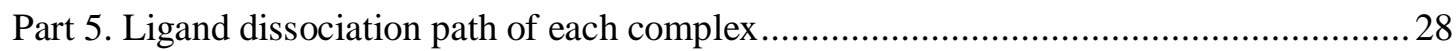

Part 6. Conformational landscapes of dynamic and static protein structures ..........................56

Part 7. A small-scale comparison of local-scaled MD and $\tau$ RAMD ....................................58

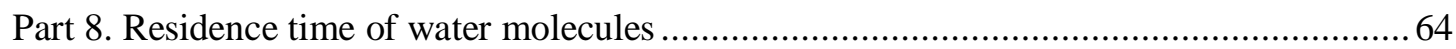




\section{Part 1. Protocols and extra results of local-scaled MD}

Table S1. The scaling protocol of local-scaled MD

\begin{tabular}{|c|c|c|c|}
\hline Time step & $\begin{array}{r}\text { The electrostatic } \\
\text { potential }\end{array}$ & $\begin{array}{r}\text { The attactive part of } \\
\text { Lennard-Jones potential }\end{array}$ & $\begin{array}{r}\text { The repulsive part of } \\
\text { Lennard-Jones potential }\end{array}$ \\
\hline $1-100$ ps & $100 \%$ & $100 \%$ & $100 \%$ \\
\hline $101-200$ ps & $97.50 \%$ & $97.50 \%$ & $100 \%$ \\
\hline $201-300$ ps & $95 \%$ & $95 \%$ & $100 \%$ \\
\hline $301-400$ ps & $92.50 \%$ & $92.50 \%$ & $100 \%$ \\
\hline $401-500$ ps & $90 \%$ & $90 \%$ & $100 \%$ \\
\hline $501-600$ ps & $87.50 \%$ & $87.50 \%$ & $100 \%$ \\
\hline $601-700$ ps & $85 \%$ & $85 \%$ & $100 \%$ \\
\hline $701-800$ ps & $82.50 \%$ & $82.50 \%$ & $100 \%$ \\
\hline $801-900$ ps & $80 \%$ & $80 \%$ & $100 \%$ \\
\hline $901-1000$ ps & $77.50 \%$ & $77.50 \%$ & $100 \%$ \\
\hline $1001-1100$ ps & $75 \%$ & $75 \%$ & $100 \%$ \\
\hline $1101-1200$ ps & $72.50 \%$ & $72.50 \%$ & $100 \%$ \\
\hline $1201-1300$ ps & $70 \%$ & $70 \%$ & $100 \%$ \\
\hline $1301-1400$ ps & $67.50 \%$ & $67.50 \%$ & $100 \%$ \\
\hline $1401-1500$ ps & $65 \%$ & $65 \%$ & $100 \%$ \\
\hline $1501-1600$ ps & $62.50 \%$ & $62.50 \%$ & $100 \%$ \\
\hline $1601-1700$ ps & $60 \%$ & $60 \%$ & $100 \%$ \\
\hline $1701-1800$ ps & $57.50 \%$ & $57.50 \%$ & $100 \%$ \\
\hline $1801-1900$ ps & $55 \%$ & $55 \%$ & $100 \%$ \\
\hline $1901-2000$ ps & $52.50 \%$ & $52.50 \%$ & $100 \%$ \\
\hline $2001-2100$ ps & $50 \%$ & $50 \%$ & $100 \%$ \\
\hline $2101-2200$ ps & $47.50 \%$ & $47.50 \%$ & $100 \%$ \\
\hline $2201-2300$ ps & $45 \%$ & $45 \%$ & $100 \%$ \\
\hline $2301-2400$ ps & $42.50 \%$ & $42.50 \%$ & $100 \%$ \\
\hline $2401-2500$ ps & $40 \%$ & $40 \%$ & $100 \%$ \\
\hline $2501-2600$ ps & $37.50 \%$ & $37.50 \%$ & $100 \%$ \\
\hline $2601-2700$ ps & $35 \%$ & $35 \%$ & $100 \%$ \\
\hline $2701-2800$ ps & $32.50 \%$ & $32.50 \%$ & $100 \%$ \\
\hline $2801-2900$ ps & $30 \%$ & $30 \%$ & $100 \%$ \\
\hline $2901-3000$ ps & $27.50 \%$ & $27.50 \%$ & $100 \%$ \\
\hline $3001-3100$ ps & $25 \%$ & $25 \%$ & $100 \%$ \\
\hline $3101-3200$ ps & $22.50 \%$ & $22.50 \%$ & $100 \%$ \\
\hline $3201-3300$ ps & $20 \%$ & $20 \%$ & $100 \%$ \\
\hline $3301-3400$ ps & $17.50 \%$ & $17.50 \%$ & $100 \%$ \\
\hline $3401-3500$ ps & $15 \%$ & $15 \%$ & $100 \%$ \\
\hline $3501-3600$ ps & $12.50 \%$ & $12.50 \%$ & $100 \%$ \\
\hline $3601-3700$ ps & $10 \%$ & $10 \%$ & $100 \%$ \\
\hline $3701-3800$ ps & $7.50 \%$ & $7.50 \%$ & $100 \%$ \\
\hline $3801-3900$ ps & $5 \%$ & $5 \%$ & $100 \%$ \\
\hline $3901-4000$ ps & $2.50 \%$ & $2.50 \%$ & $100 \%$ \\
\hline $4001-4100$ ps & $0 \%$ & $0 \%$ & $100 \%$ \\
\hline $4101-4200$ ps & $0 \%$ & $0 \%$ & $100 \%$ \\
\hline $4201-4300$ ps & $0 \%$ & $0 \%$ & $100 \%$ \\
\hline $4301-4400$ ps & $0 \%$ & $0 \%$ & $100 \%$ \\
\hline $4401-4500$ ps & $0 \%$ & $0 \%$ & $100 \%$ \\
\hline $4501-4600$ ps & $0 \%$ & $0 \%$ & $100 \%$ \\
\hline $4601-4700$ ps & $0 \%$ & $0 \%$ & $100 \%$ \\
\hline $4701-4800$ ps & $0 \%$ & $0 \%$ & $100 \%$ \\
\hline $4801-4900$ ps & $0 \%$ & $0 \%$ & $100 \%$ \\
\hline $4901-5000$ ps & $0 \%$ & $0 \%$ & $100 \%$ \\
\hline
\end{tabular}



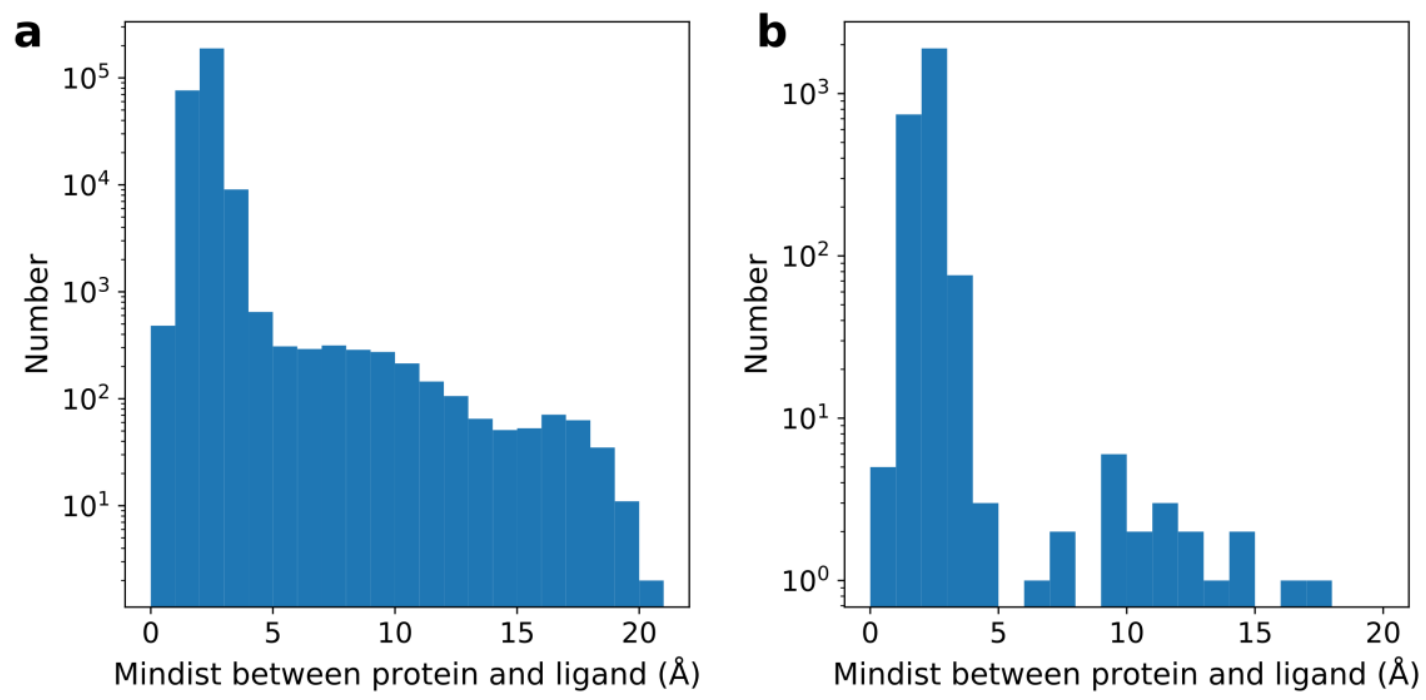

Figure S1. The mindist between protein and ligand in (a) the last 100 ps and (b) the last frame of local-scaled MD. The distance threshold is evaluated once per $100 \mathrm{ps}$ in the local-scaled MD. 


\section{Part 2. Detailed information of the kinase data set}

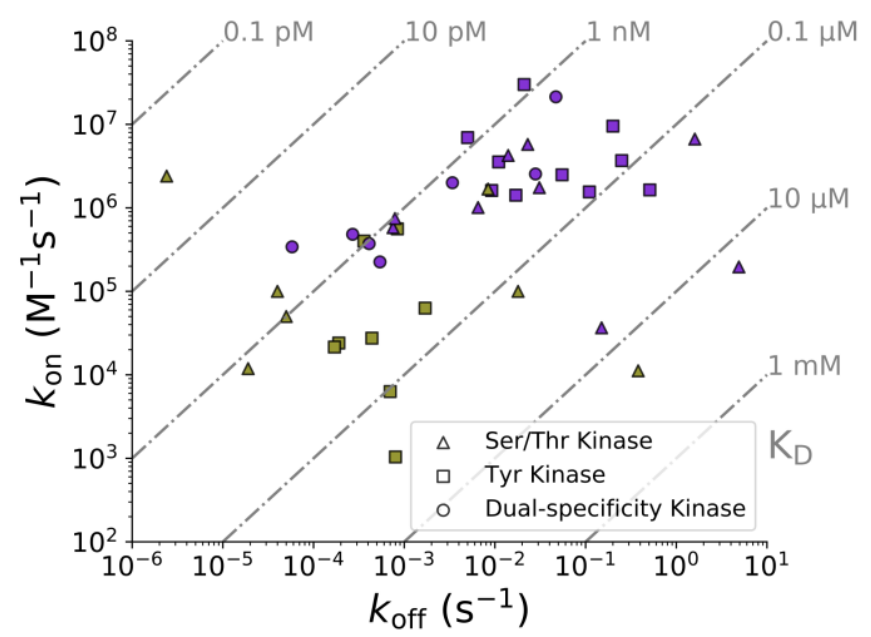

Figure S2. The iso-affinity diagonal plot indicating the kinetic constants of the 41 kinase-inhibitor complexes in our data set. The kinetic constants ( $k_{\text {on }}$ and $k_{\text {off }}$ ) were measured by surface plasmon resonance. Complexes locating on the same iso-affinity diagonal line have identical equilibrium dissociation constants $\left(K_{\mathrm{D}}\right)$. Triangle, square, and circle data points represent different kinds of protein kinases. 
Table S2. Chemical structures of ligands

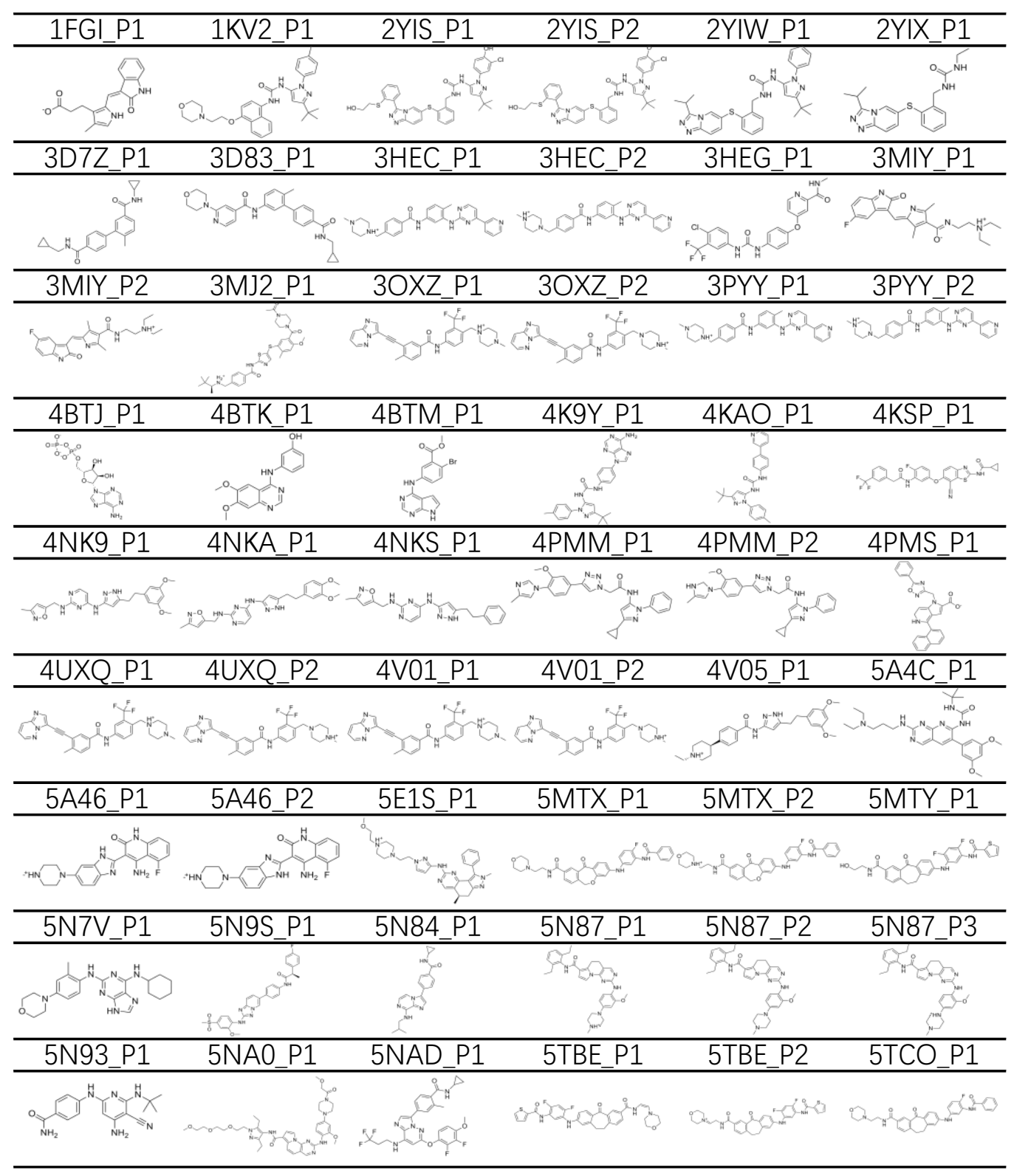




\section{Part 3. Changes of binding energy during local-scaled MD}

Figure S3. The binding energy changed over time. If the inhibitor was $9 \AA$ far away from the kinase, the simulation would stop before it reached the maximum time limit ( $5 \mathrm{~ns})$. Each trajectory is represented by a different color here.
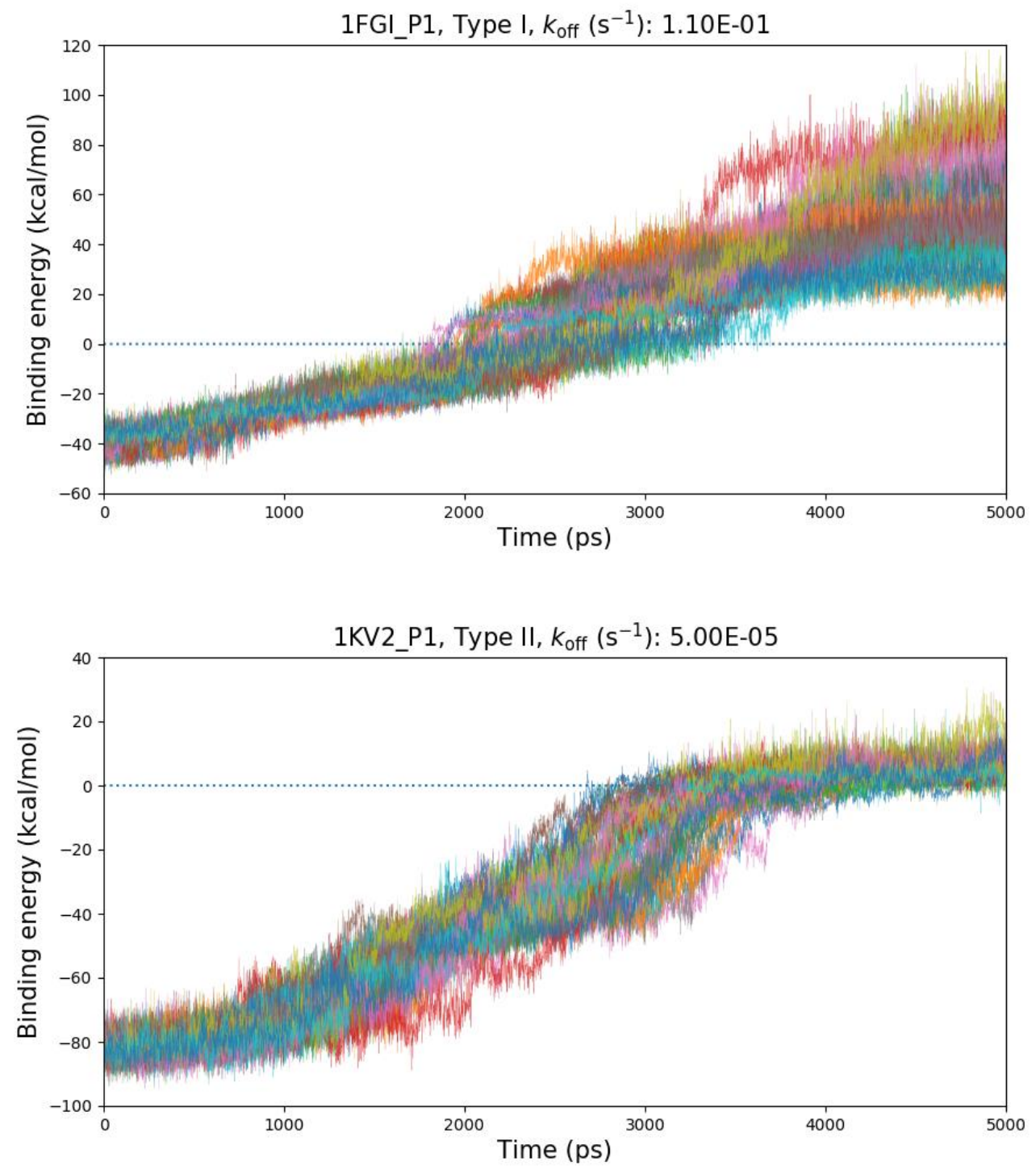

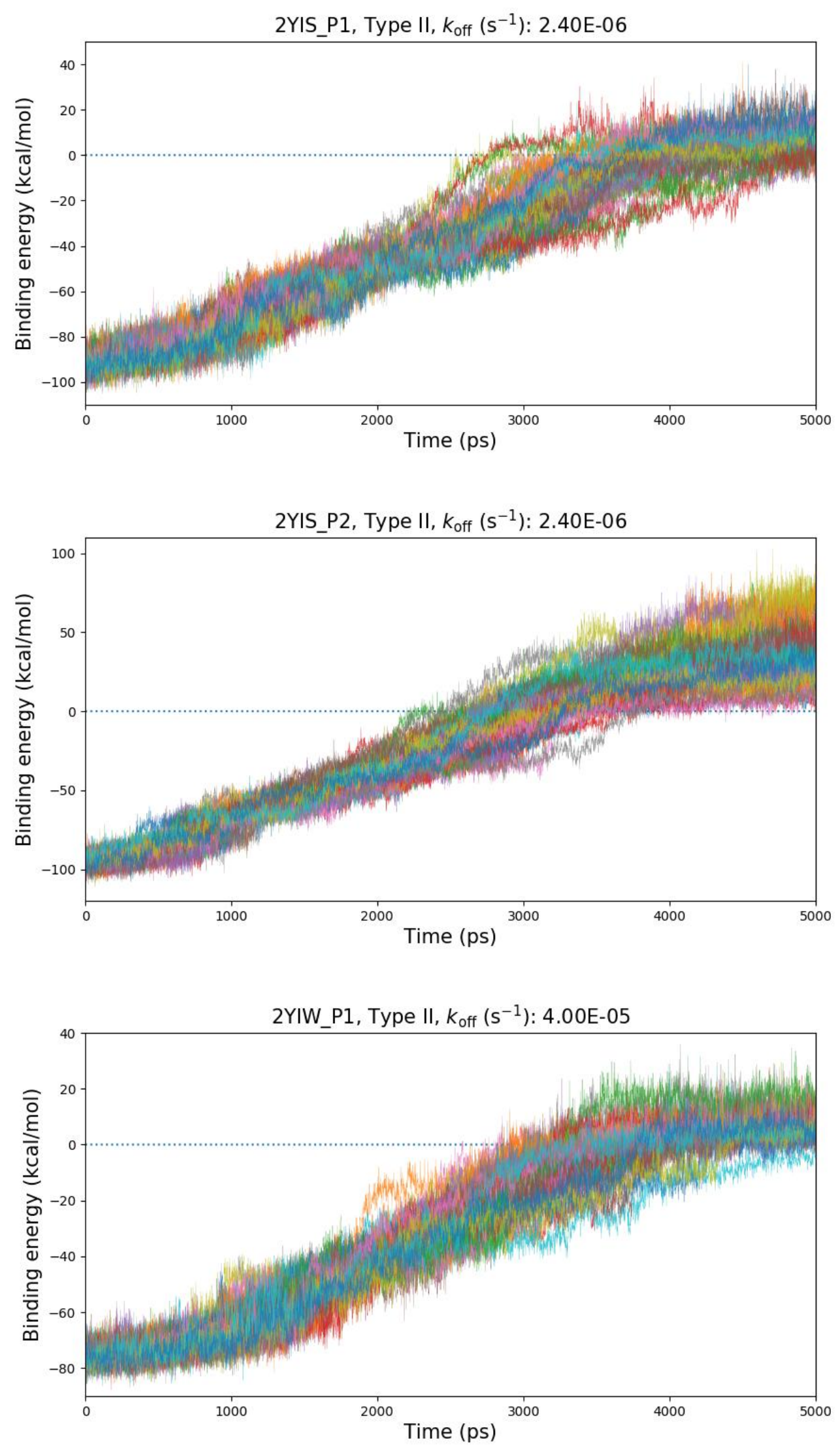

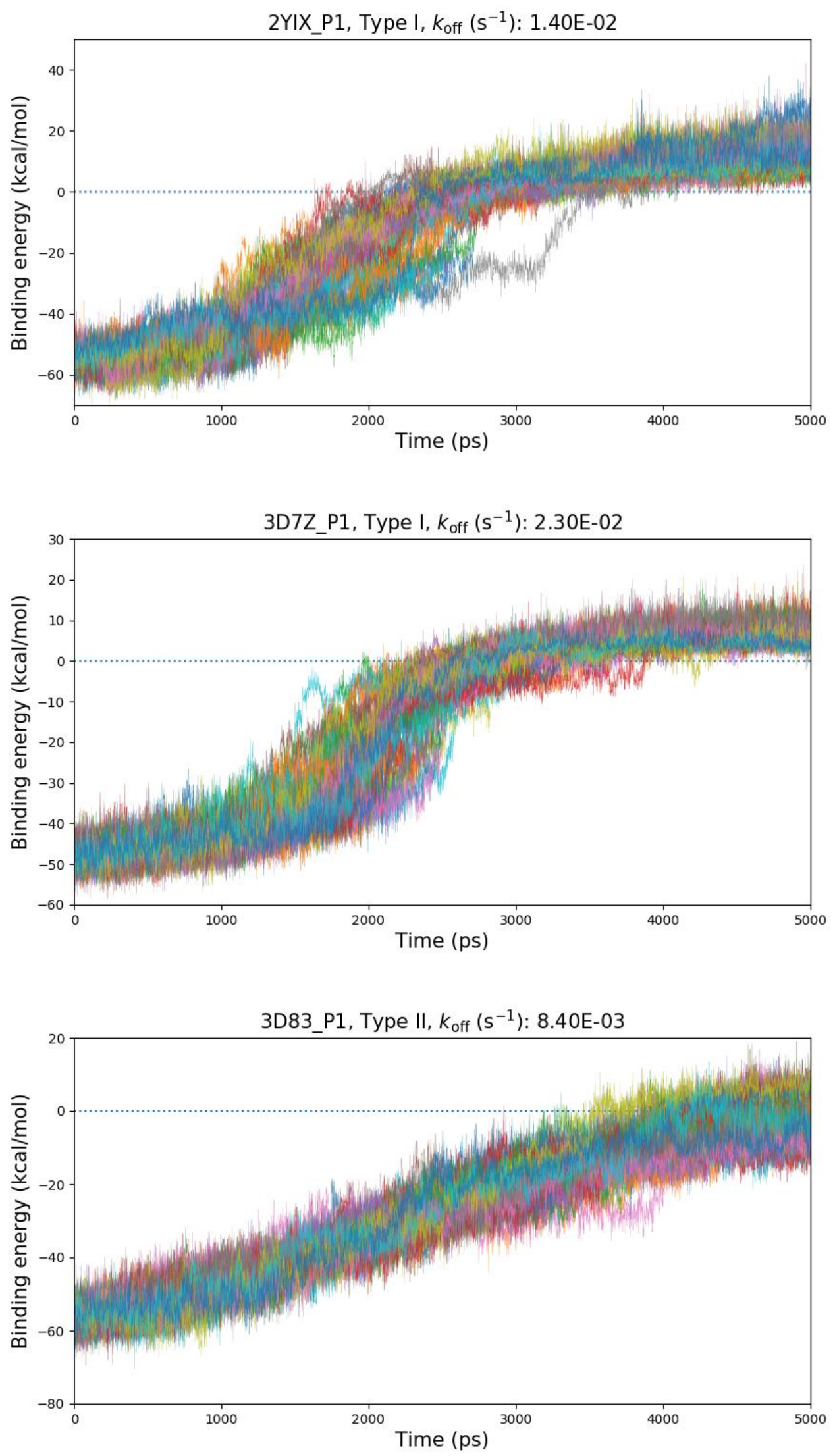
3HEC_P1, Type II, $k_{\text {off }}\left(\mathrm{s}^{-1}\right): 3.80 \mathrm{E}-01$

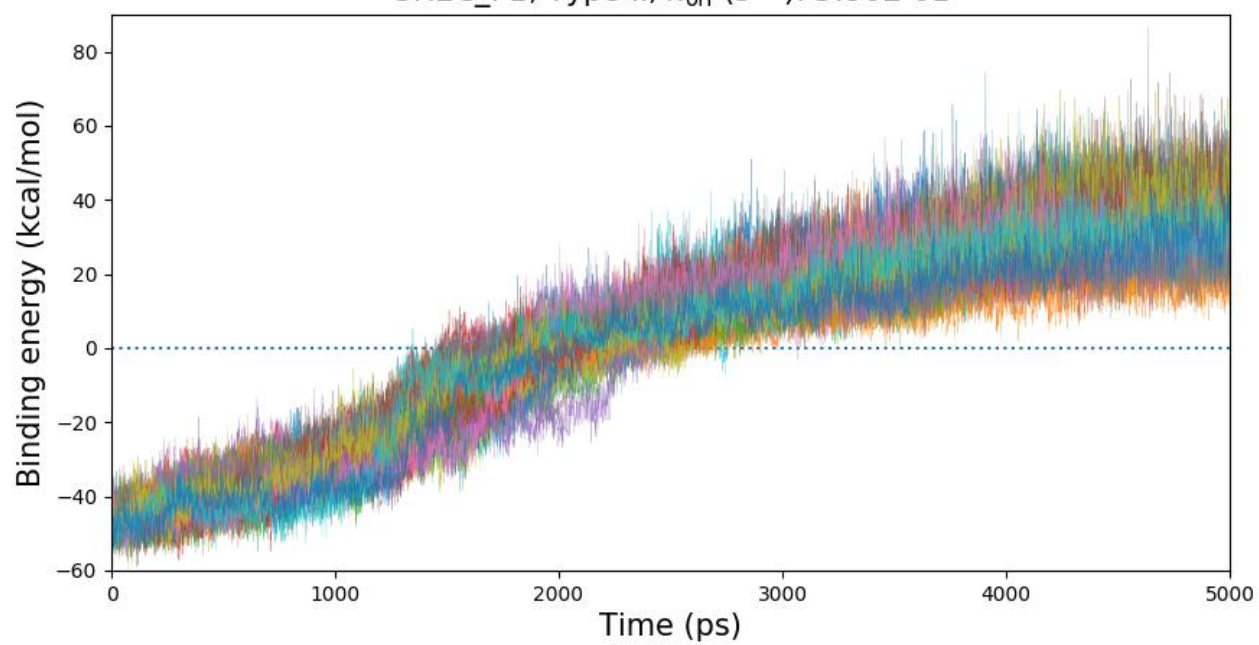

3HEC_P2, Type II, $k_{\text {off }}\left(\mathrm{s}^{-1}\right): 3.80 \mathrm{E}-01$

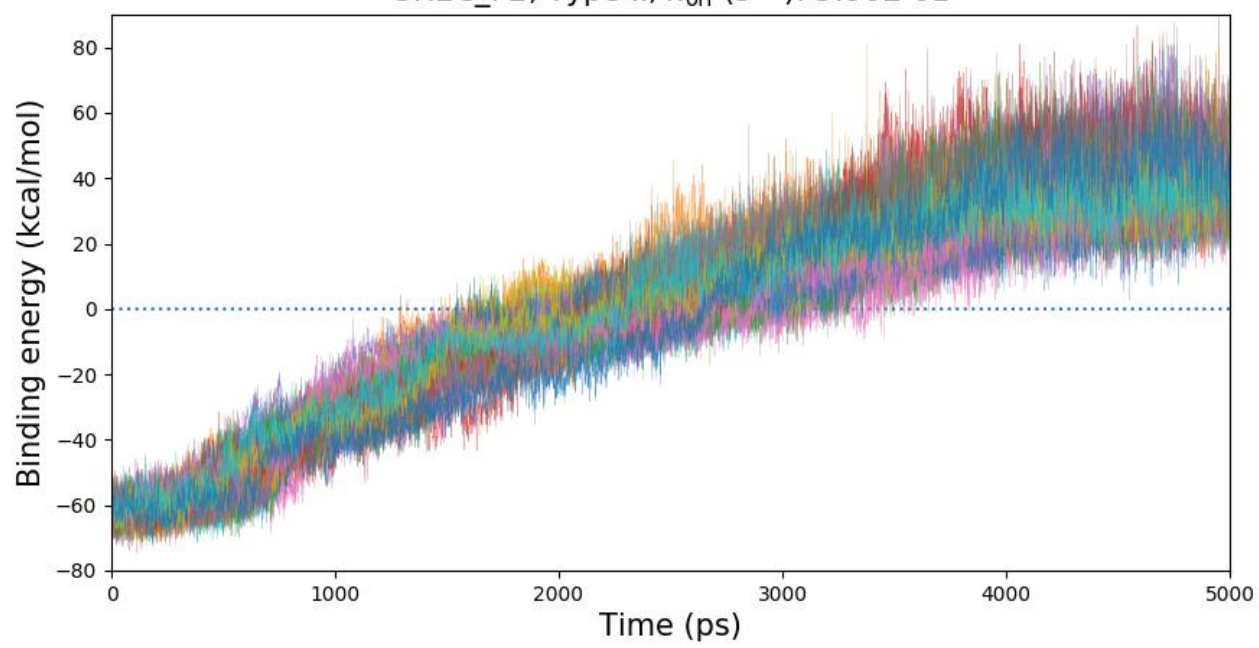

3HEG_P1, Type II, $k_{\text {off }}\left(\mathrm{s}^{-1}\right): 1.80 \mathrm{E}-02$

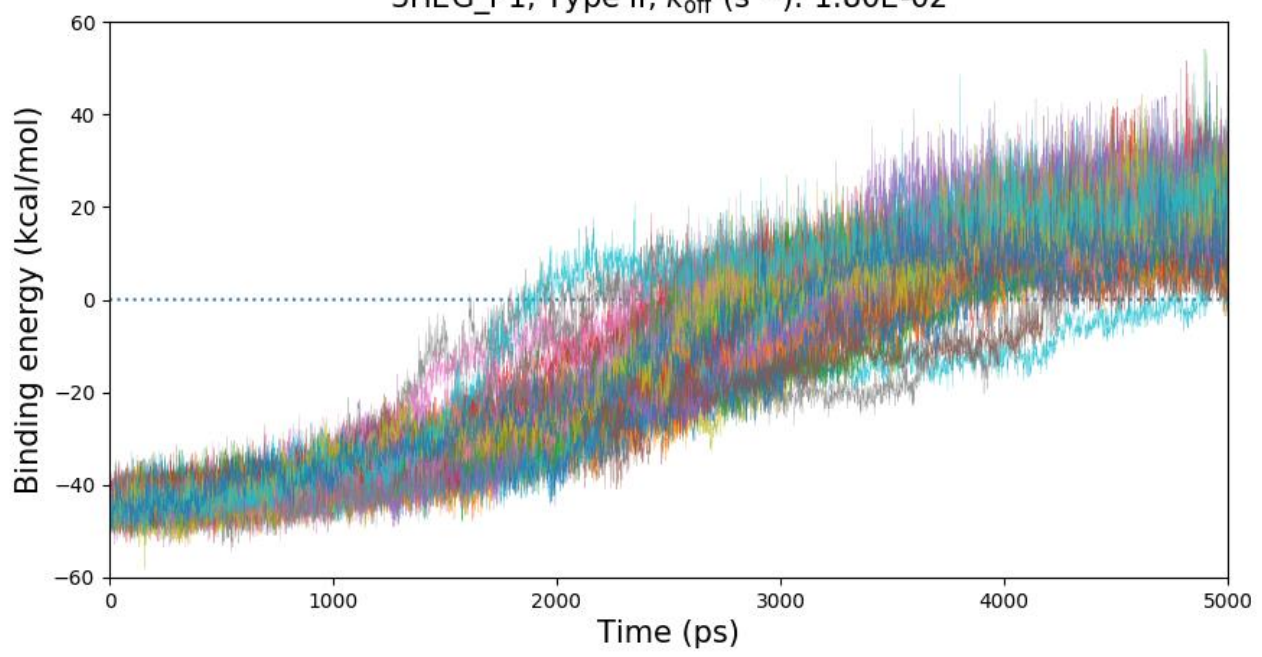



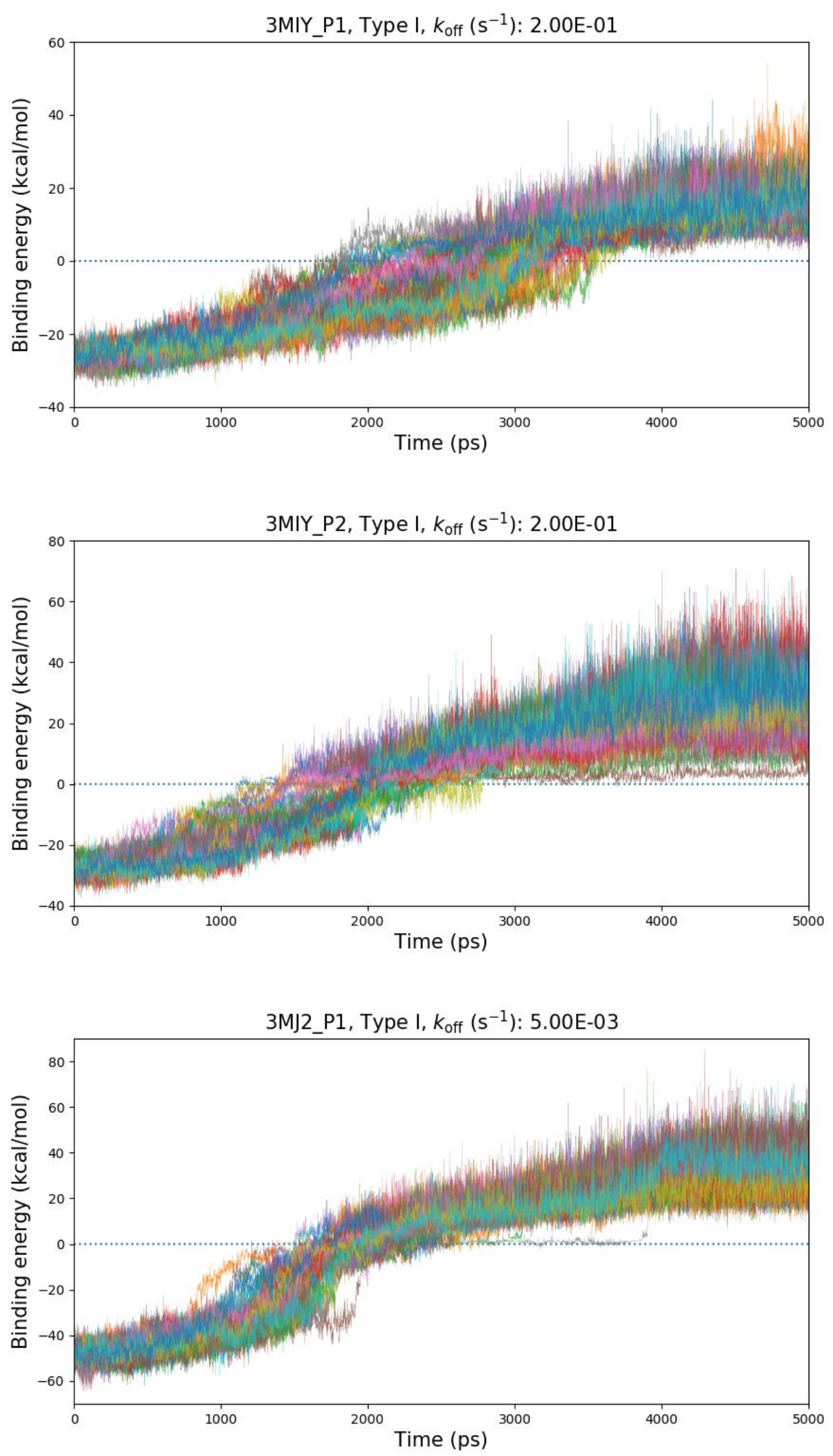
30XZ_P1, Type II, $k_{\text {off }}\left(s^{-1}\right): 3.60 E-04$

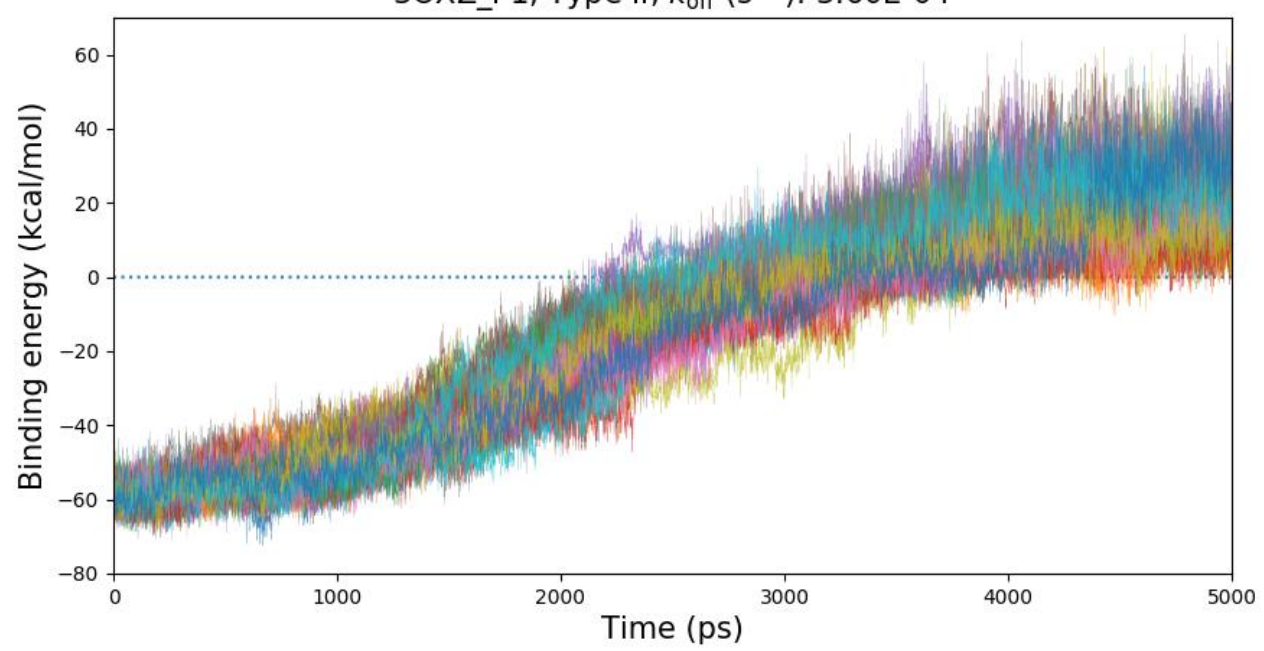

30XZ_P2, Type II, $k_{\text {off }}\left(s^{-1}\right): 3.60 E-04$

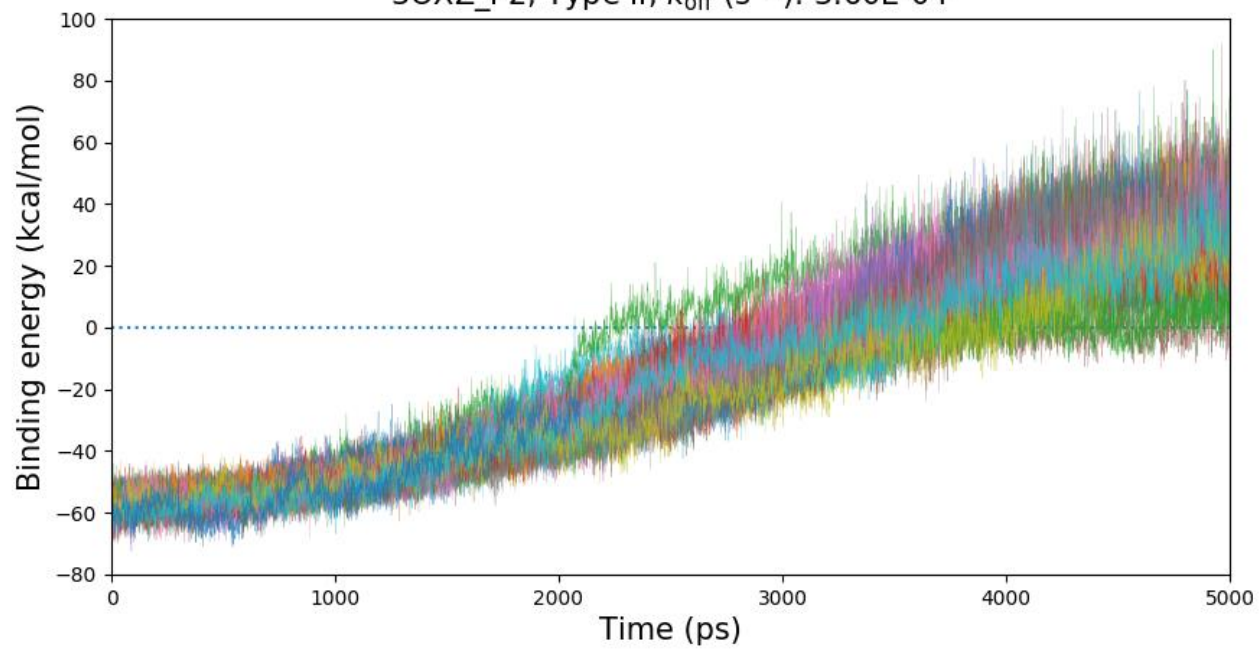

3PYY_P1, Type II, $k_{\text {off }}\left(s^{-1}\right): 8.40 E-04$

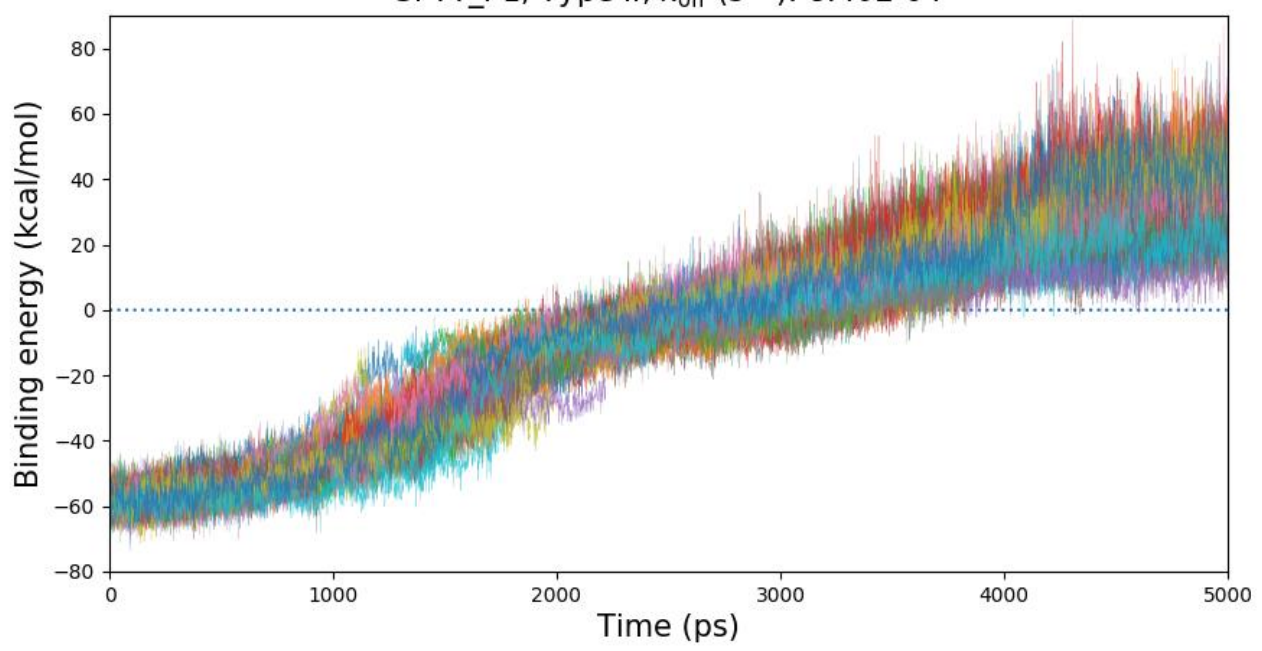



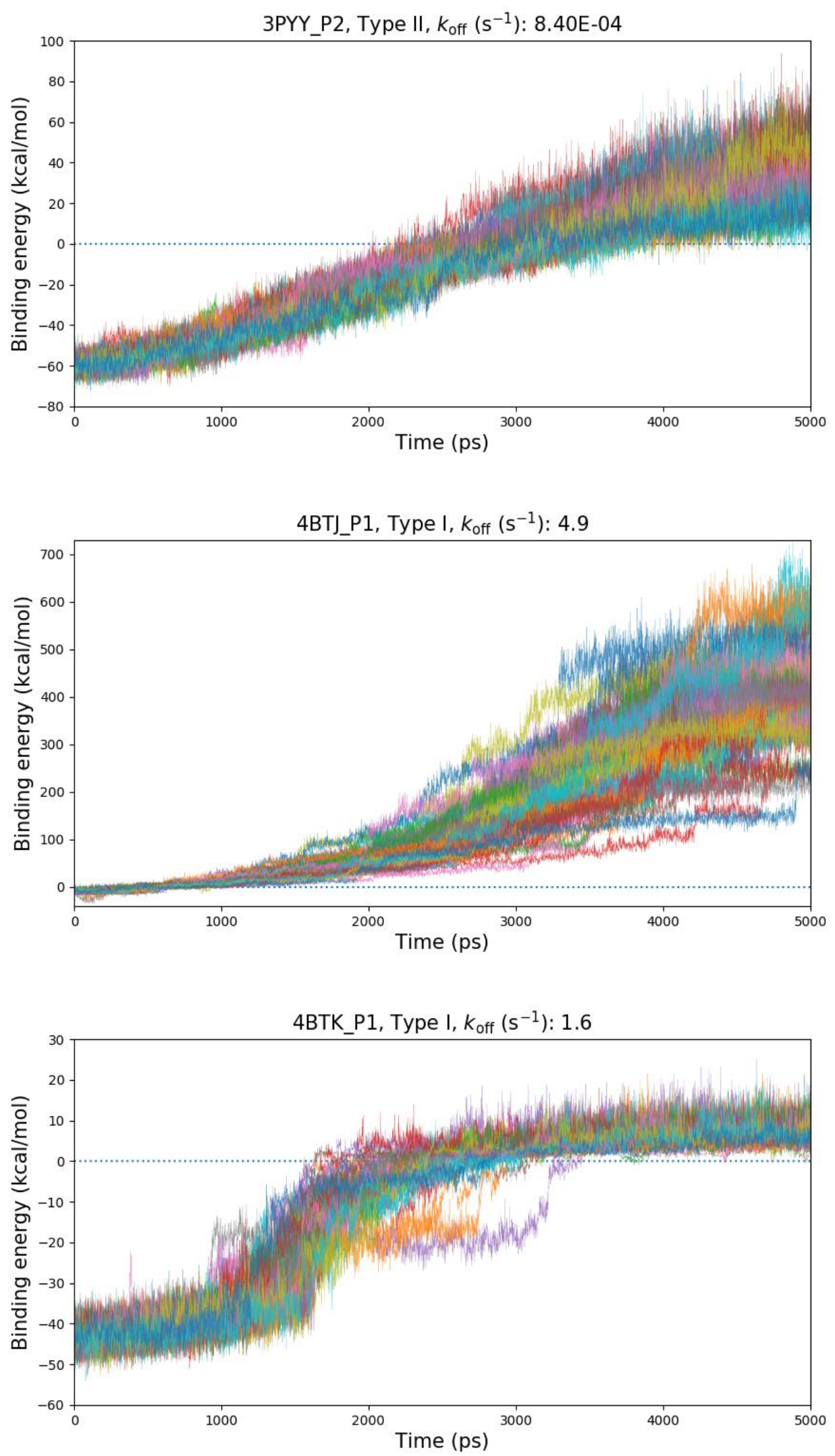

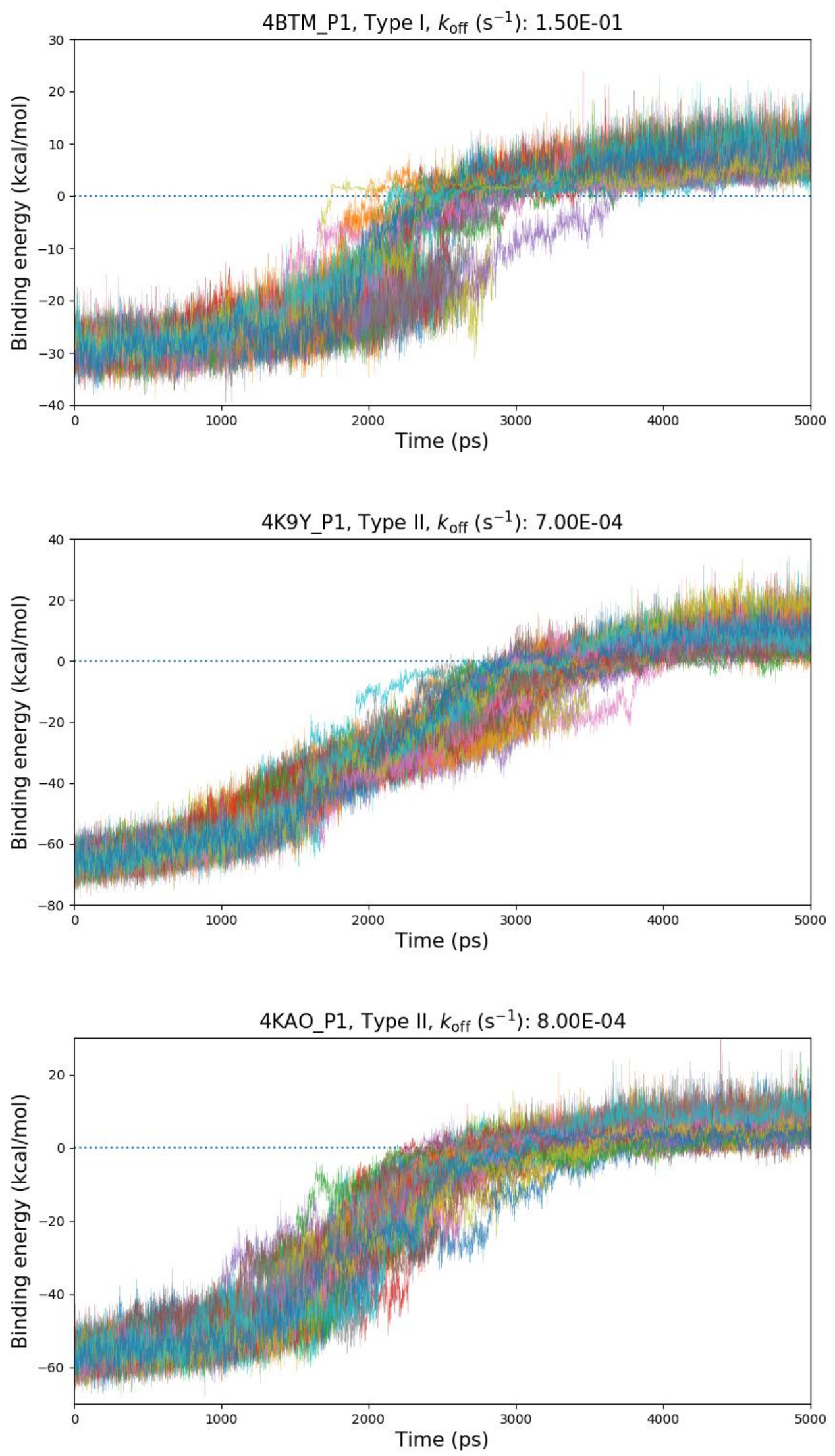

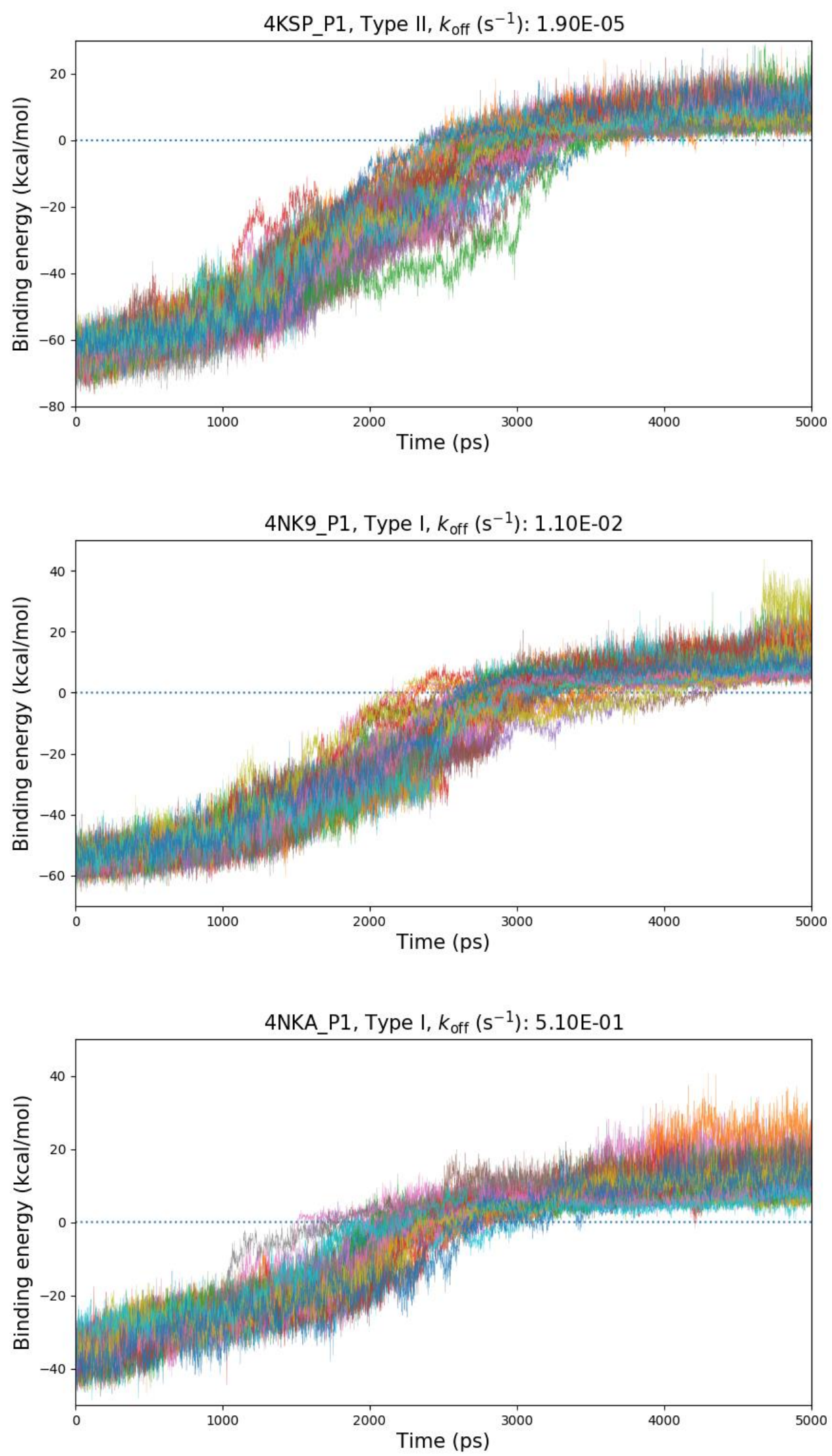
4NKS_P1, Type I, $k_{\text {off }}\left(\mathrm{s}^{-1}\right): 2.50 \mathrm{E}-01$

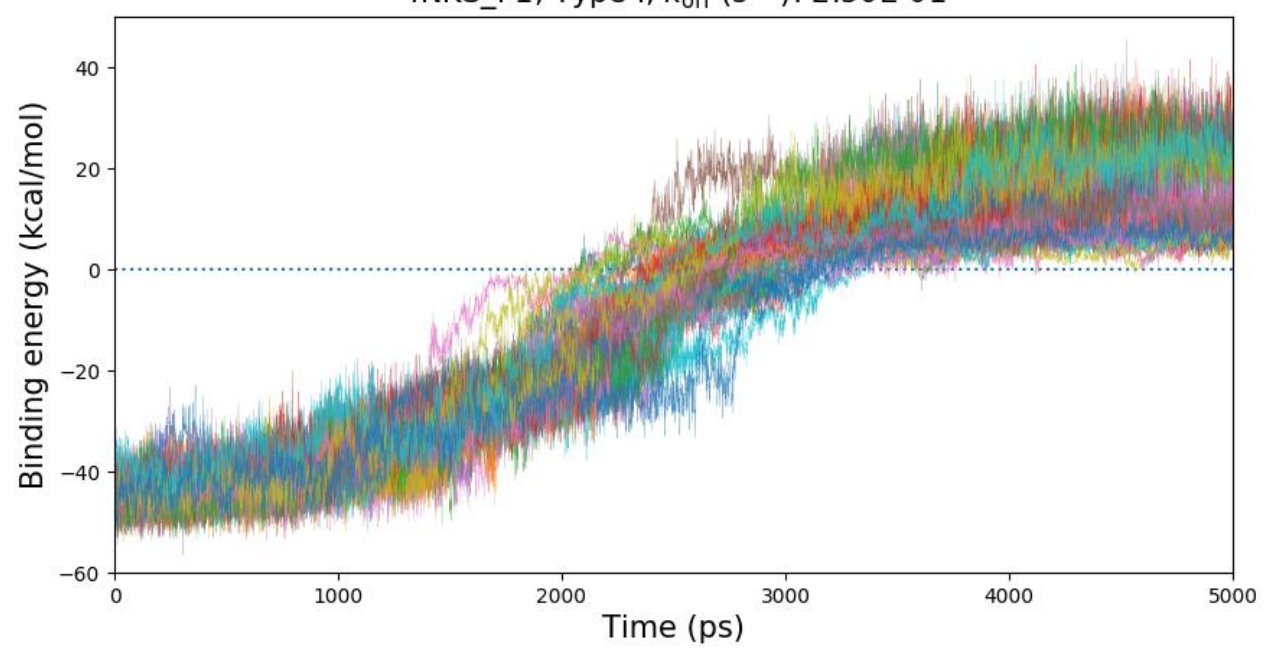

4PMM_P1, Type II, $k_{\text {off }}\left(\mathrm{s}^{-1}\right): 1.70 \mathrm{E}-03$

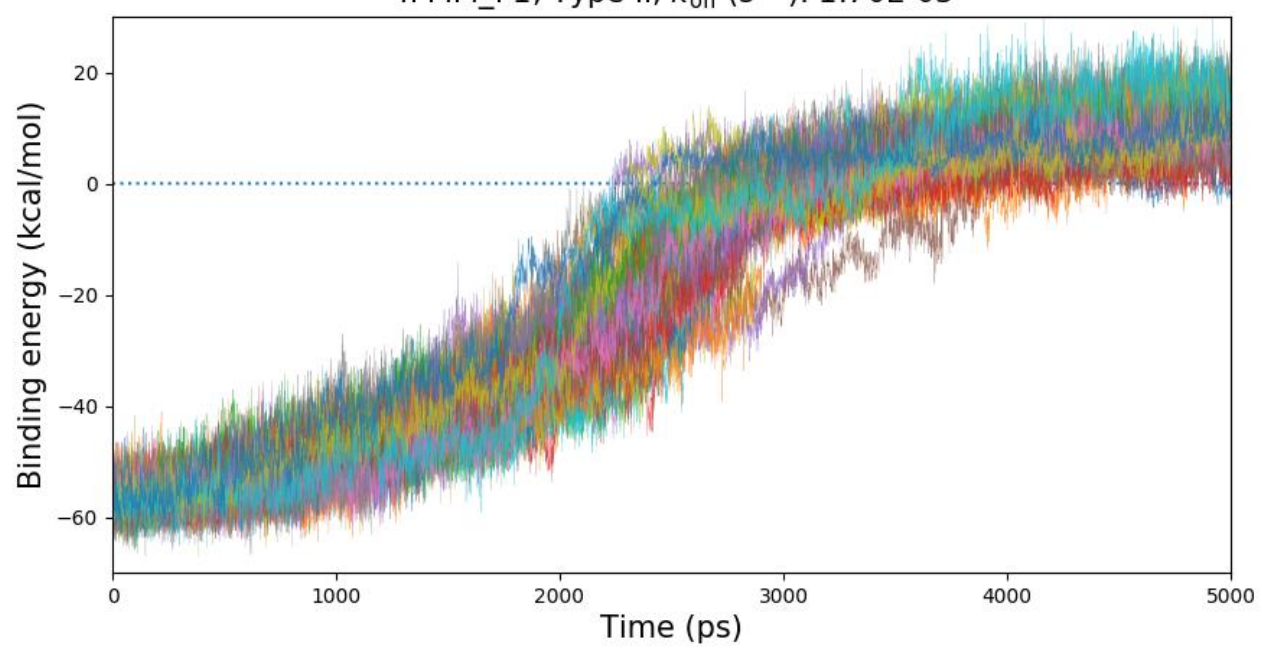

4PMM_P2, Type II, $k_{\text {off }}\left(\mathrm{s}^{-1}\right): 1.70 \mathrm{E}-03$

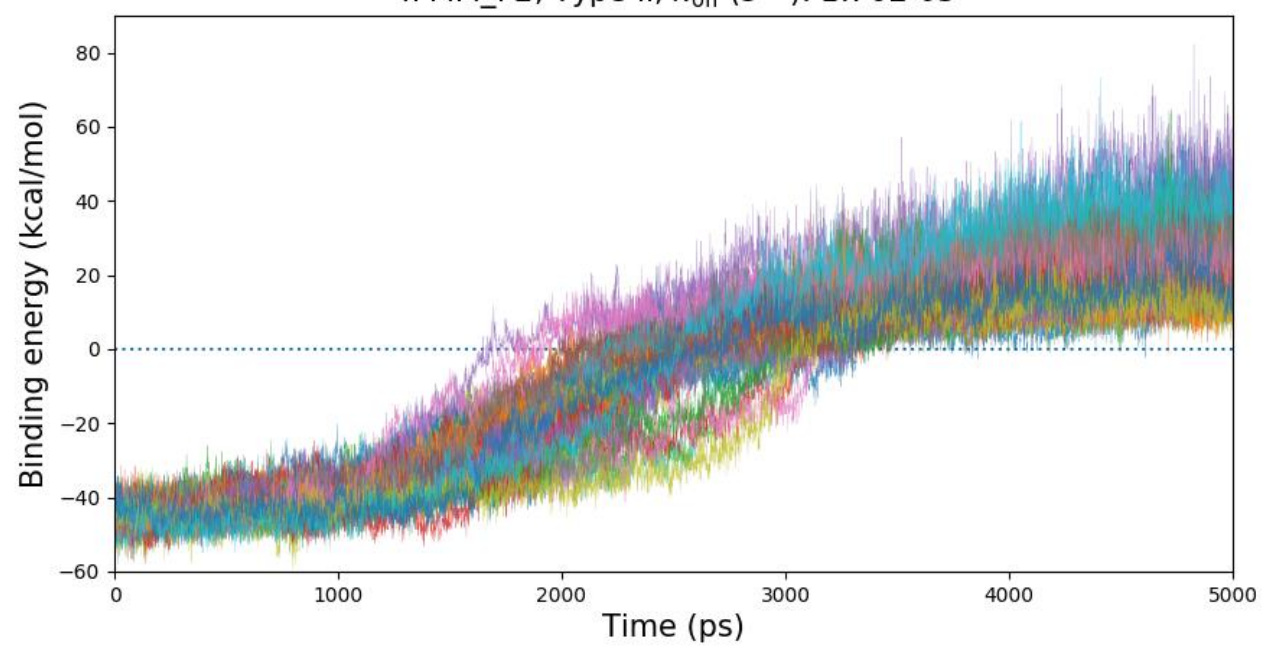



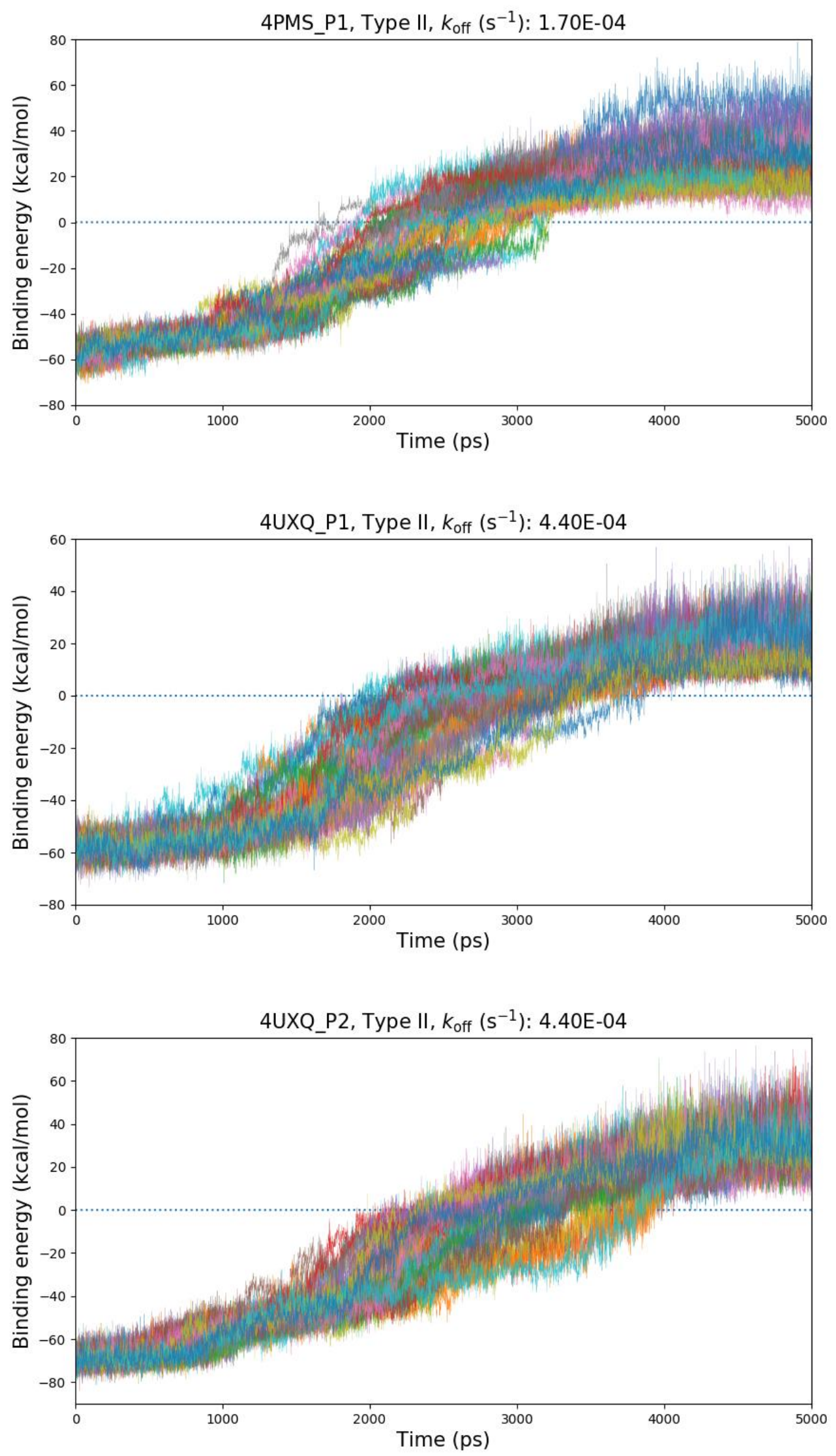
4V01_P1, Type II, $k_{\text {off }}\left(\mathrm{s}^{-1}\right): 1.90 \mathrm{E}-04$

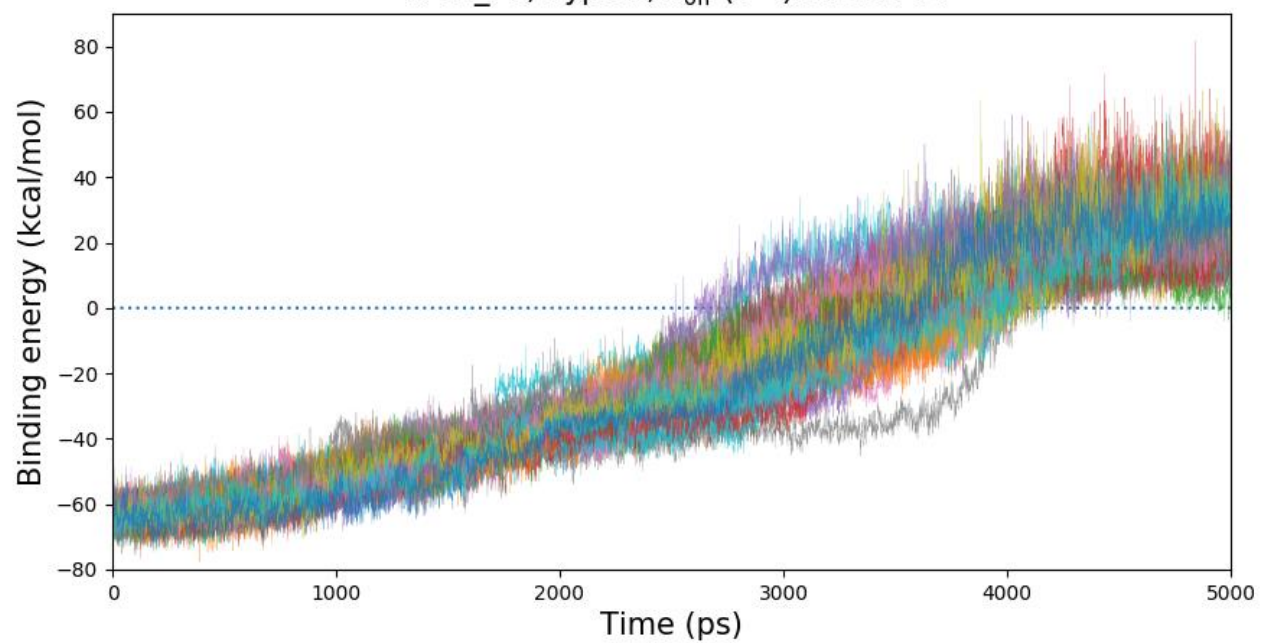

4V01_P2, Type II, $k_{\text {off }}\left(\mathrm{s}^{-1}\right): 1.90 \mathrm{E}-04$

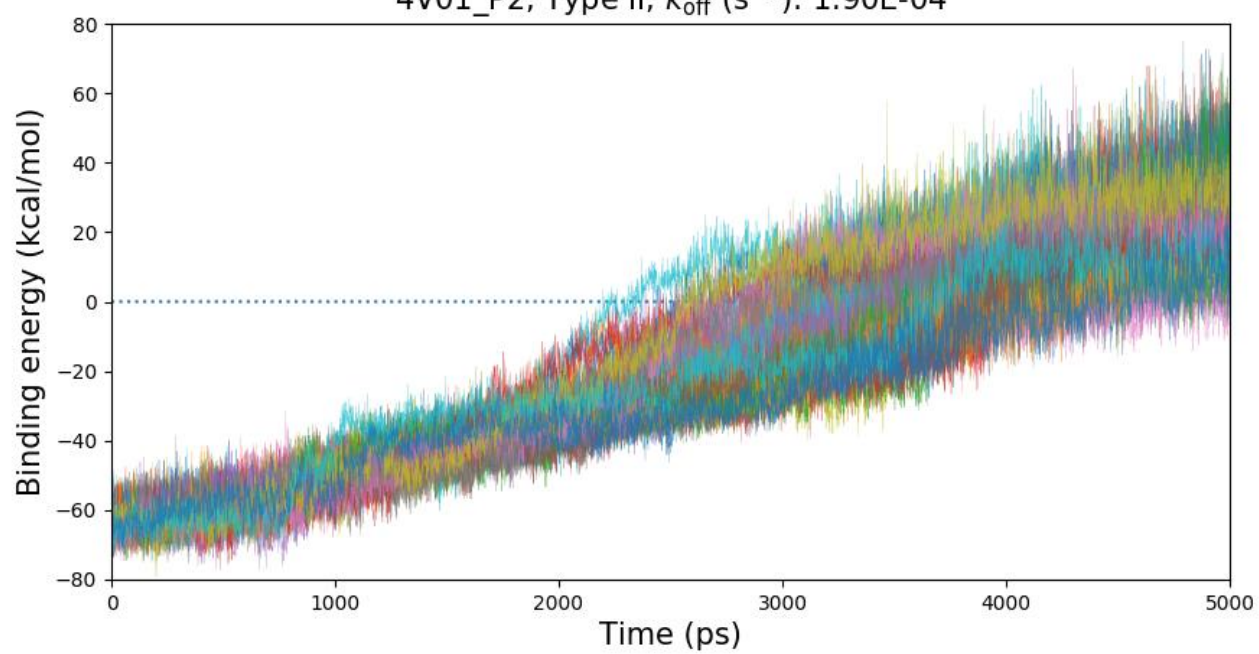

4V05_P1, Type I, $k_{\text {off }}\left(s^{-1}\right): 2.10 E-02$

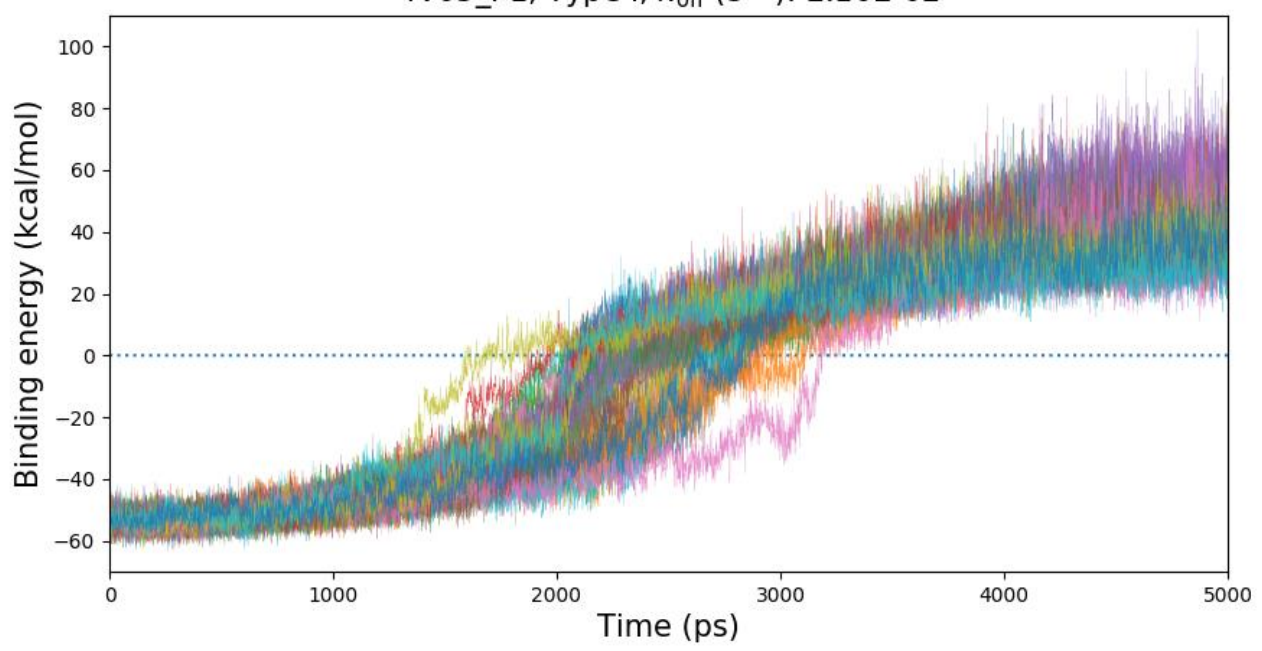



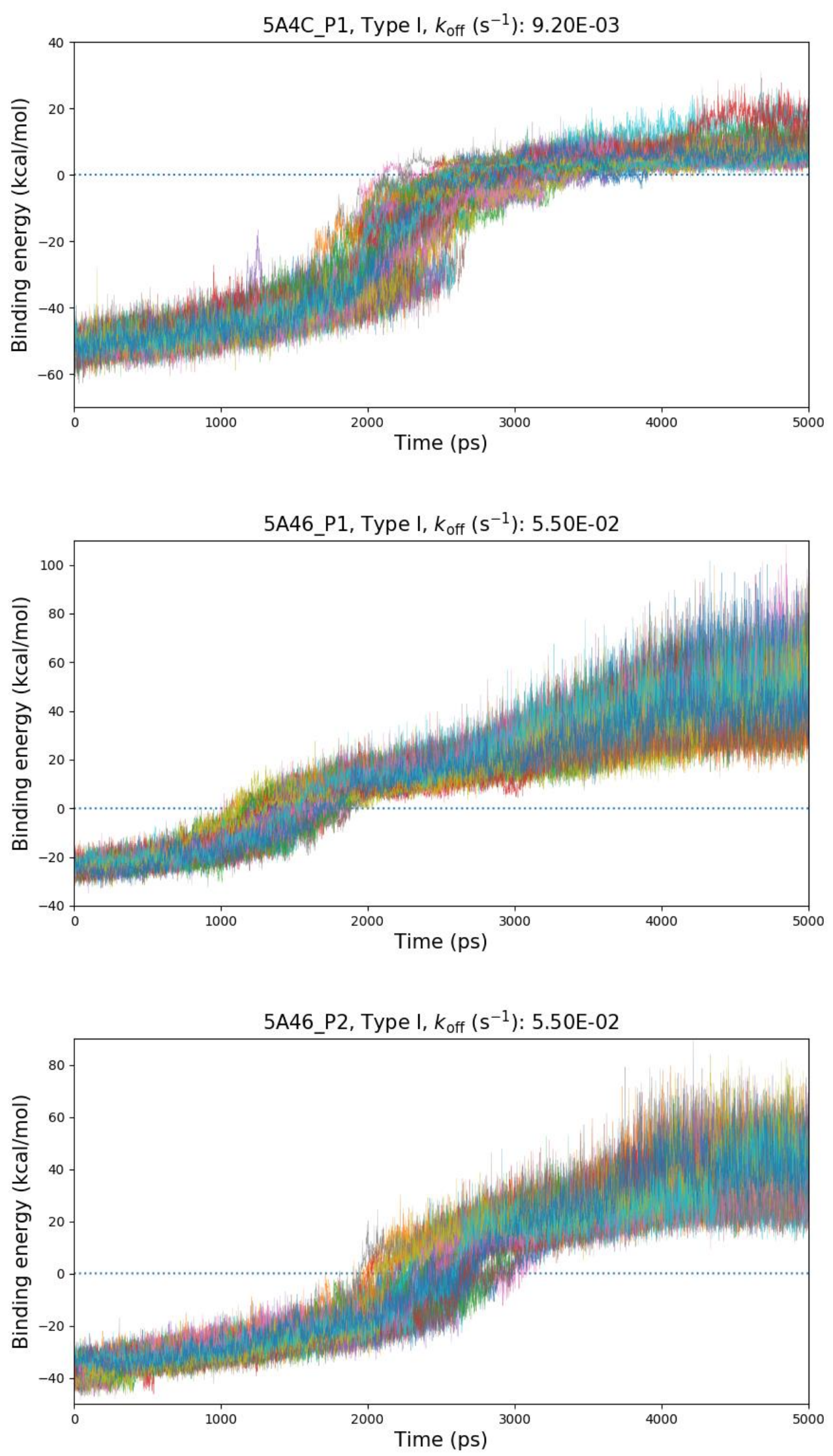
5E1S_P1, Type I, $k_{\text {off }}\left(s^{-1}\right): 1.70 E-02$

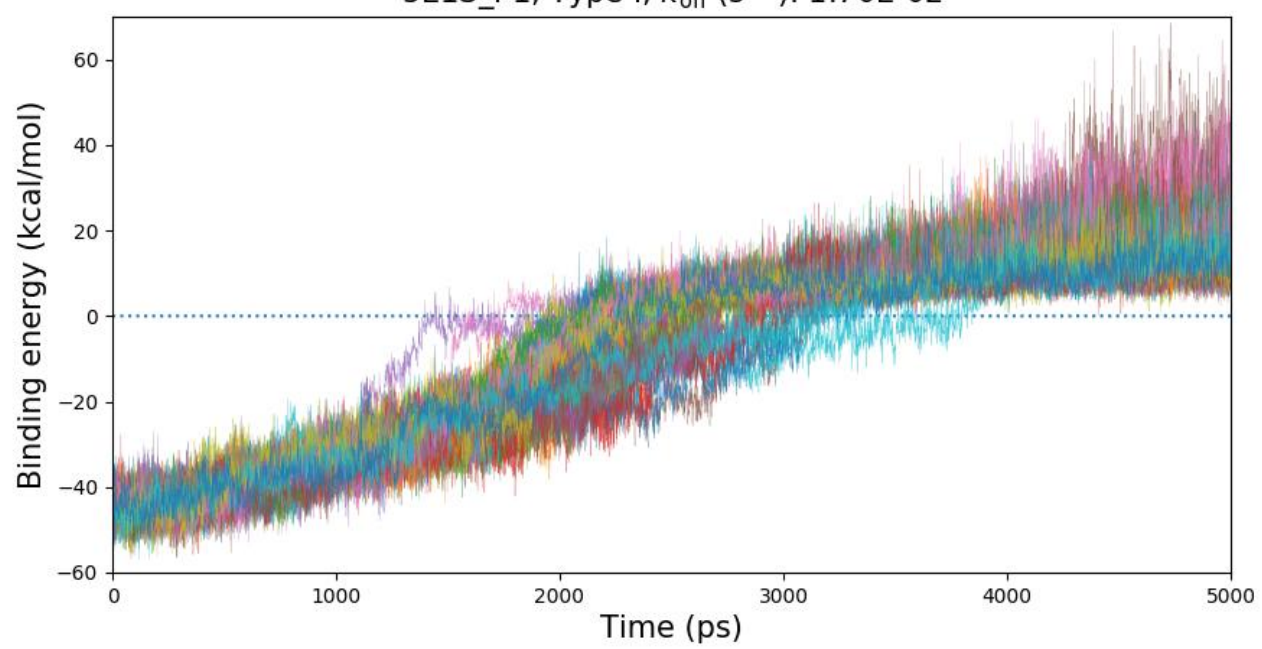

5MTX_P1, Type I, $k_{\text {off }}\left(s^{-1}\right): 3.09 E-02$

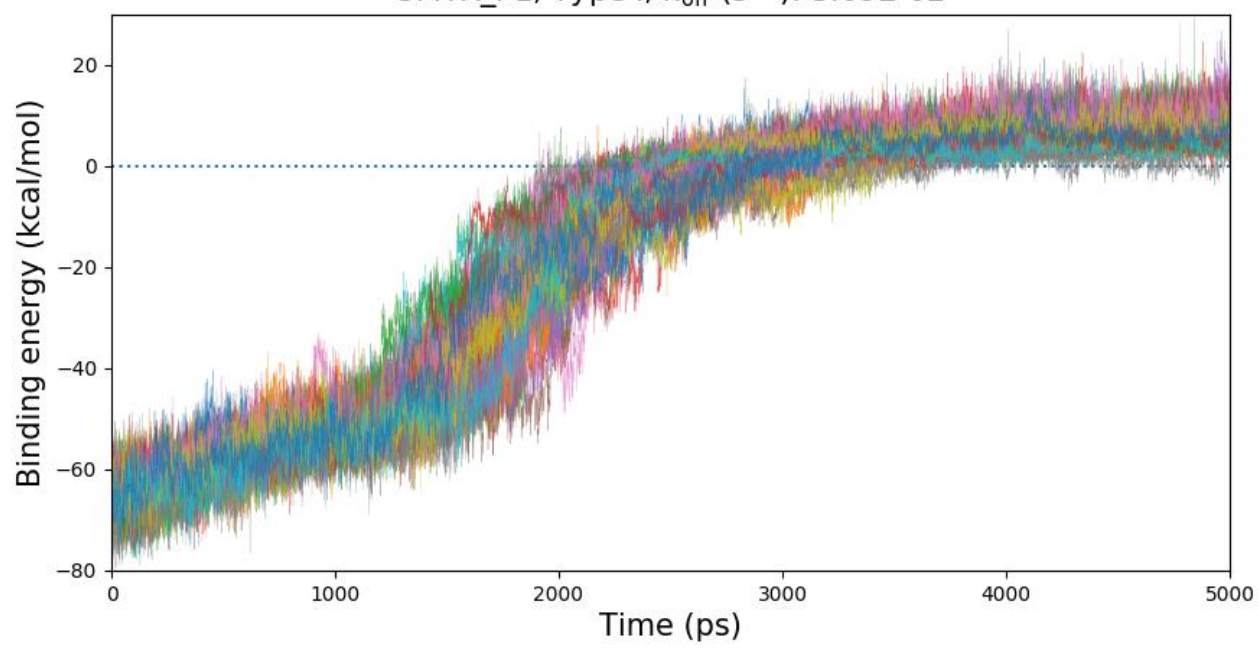

5MTX_P2, Type I, $k_{\text {off }}\left(s^{-1}\right): 3.09 E-02$

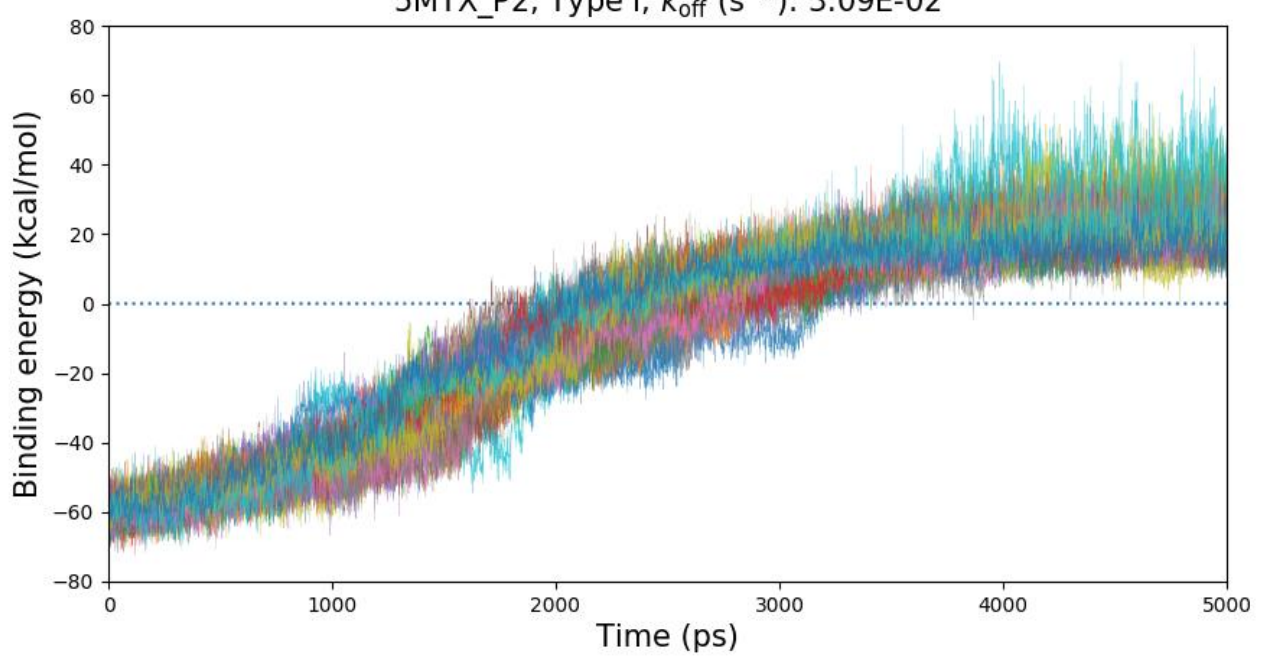


5MTY_P1, Type I, $k_{\text {off }}\left(s^{-1}\right): 7.53 E-04$

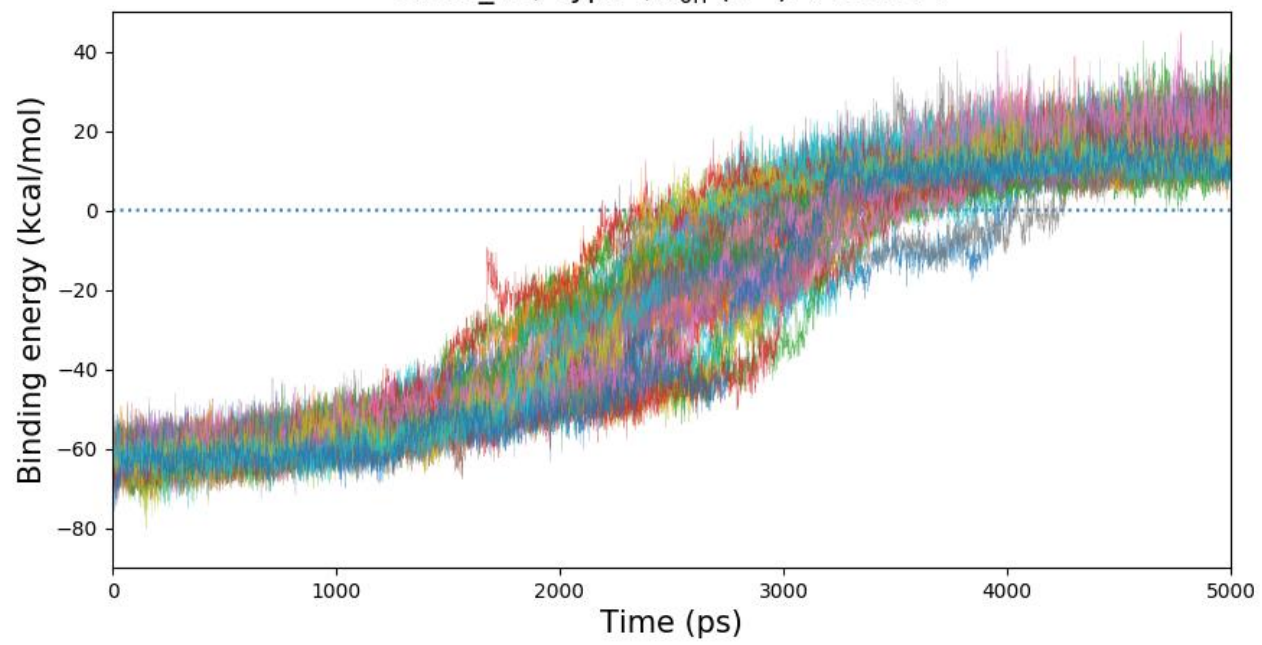

5N7V_P1, Type I, $k_{\text {off }}\left(s^{-1}\right): 3.40 E-03$

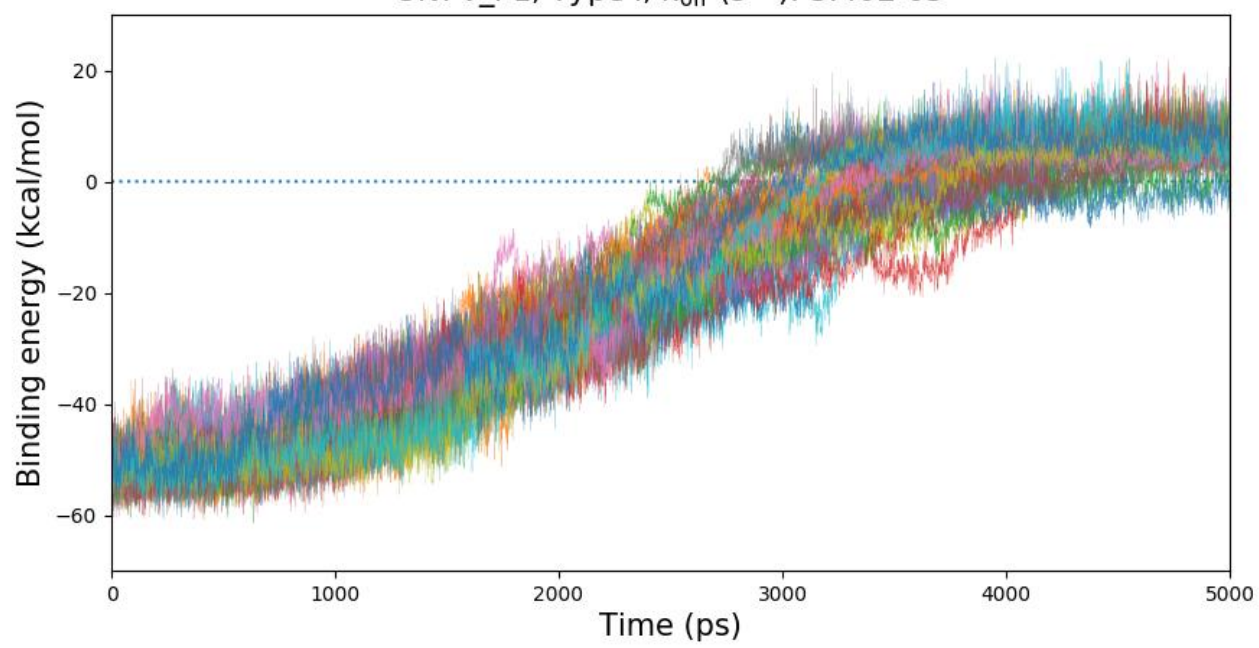

5N9S_P1, Type I, $k_{\text {off }}\left(s^{-1}\right): 5.40 E-04$

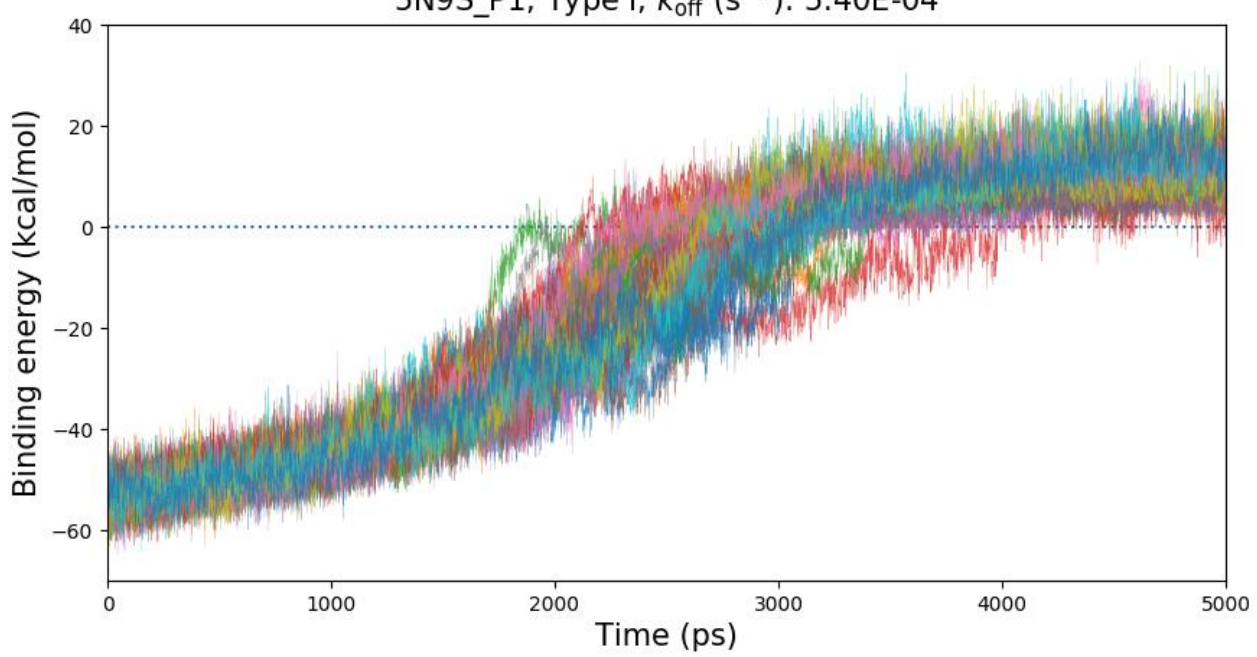



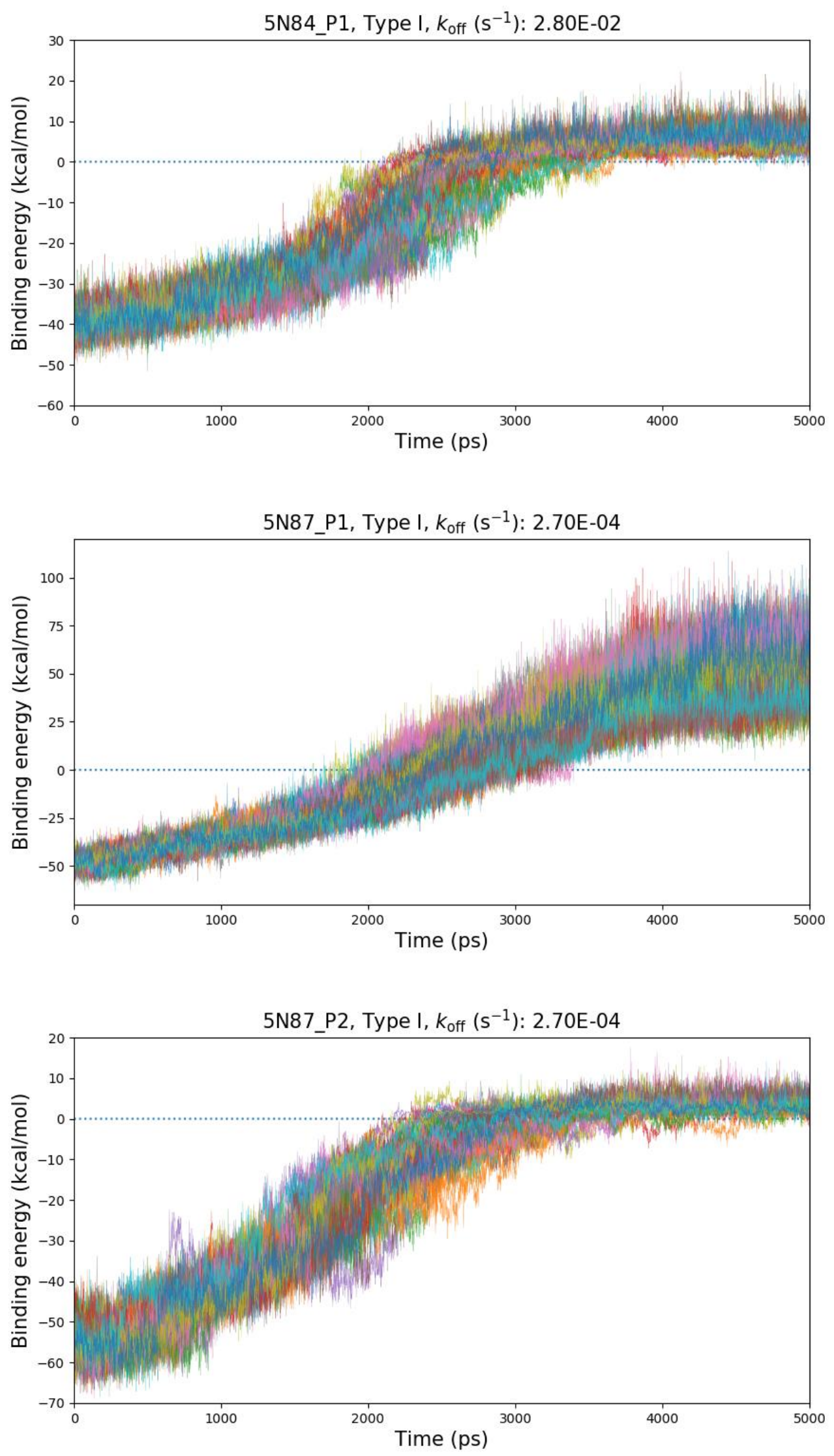

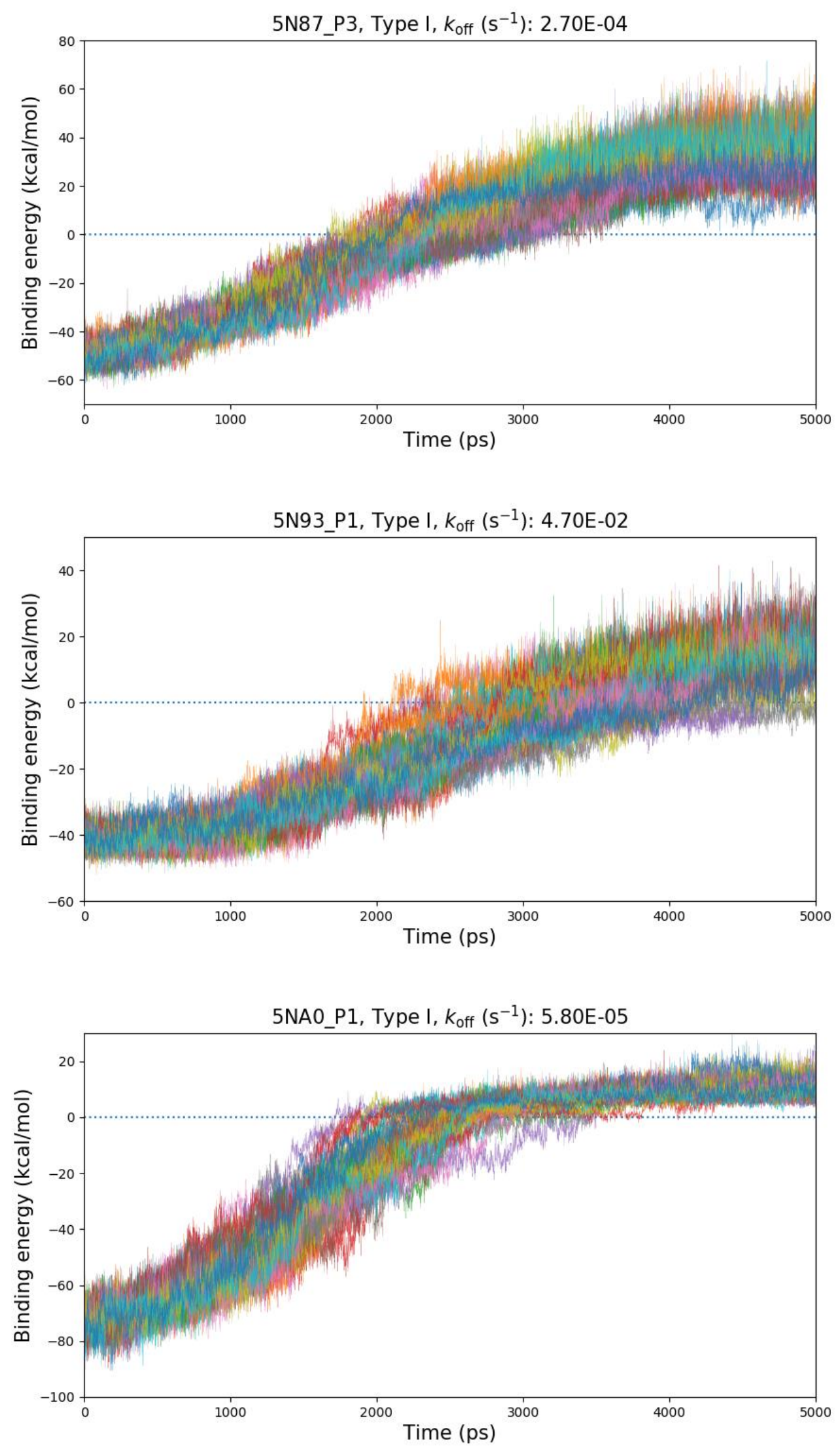
5NAD_P1, Type I, $k_{\text {off }}\left(s^{-1}\right): 4.10 E-04$

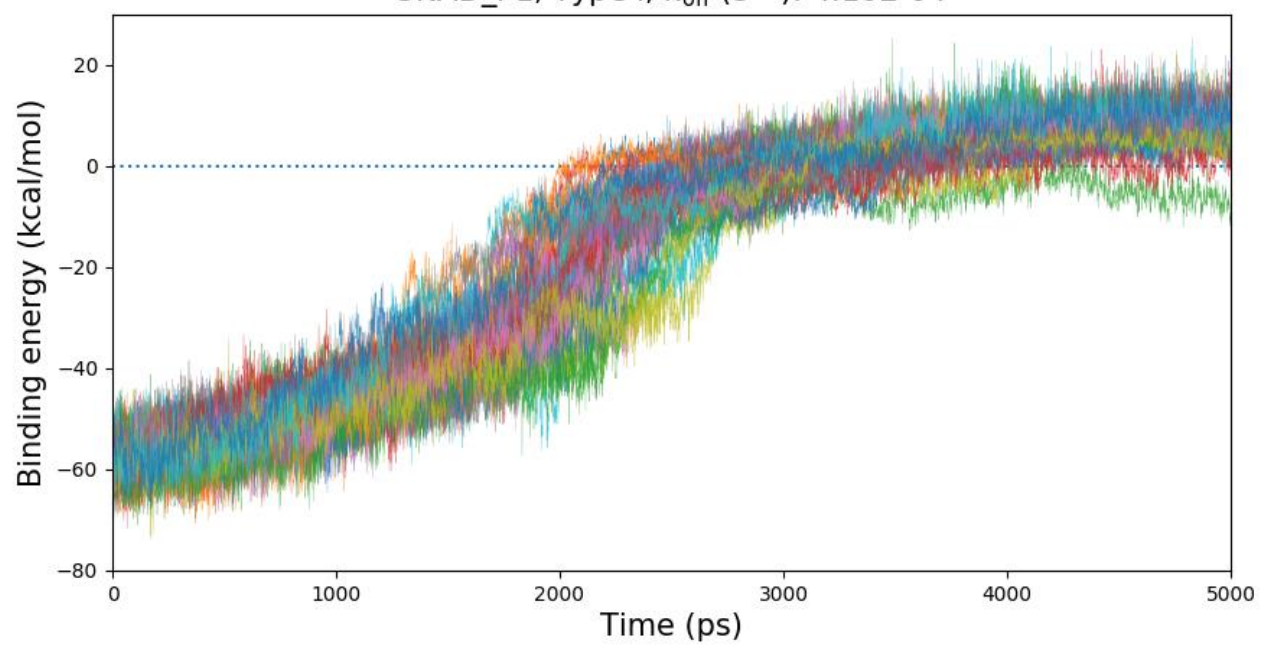

5TBE_P1, Type I, $k_{\text {off }}\left(\mathrm{s}^{-1}\right): 7.90 \mathrm{E}-04$

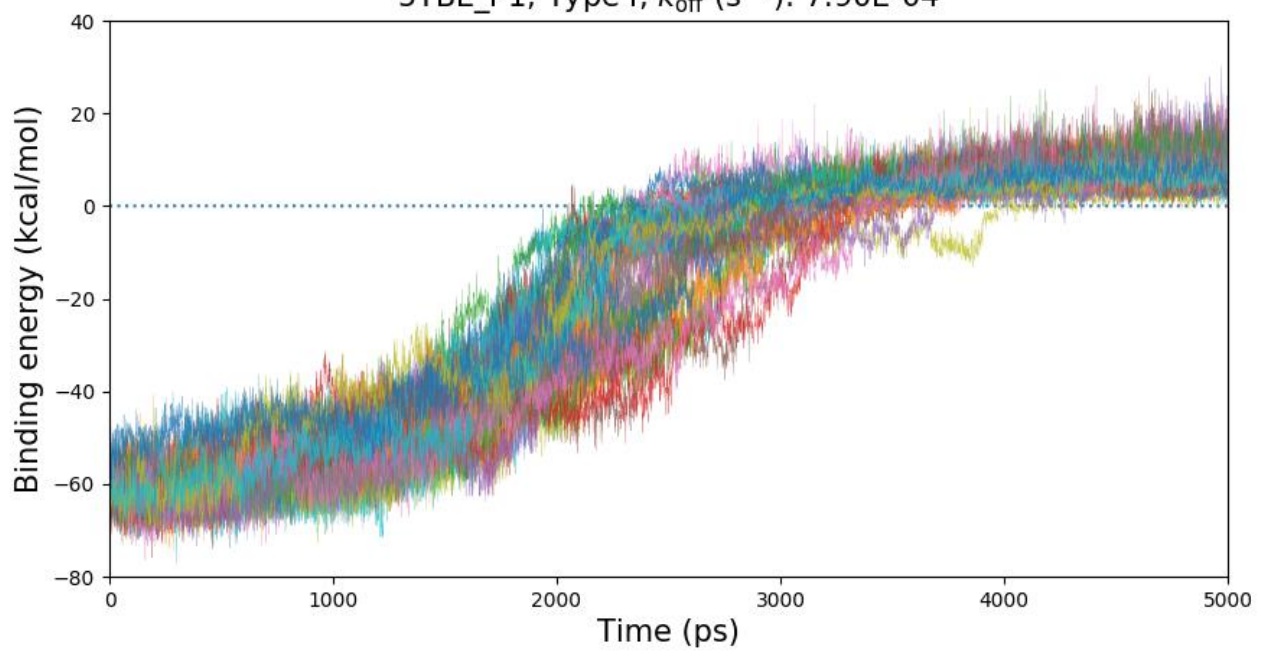


5TBE_P2, Type I, $k_{\text {off }}\left(s^{-1}\right): 7.90 E-04$
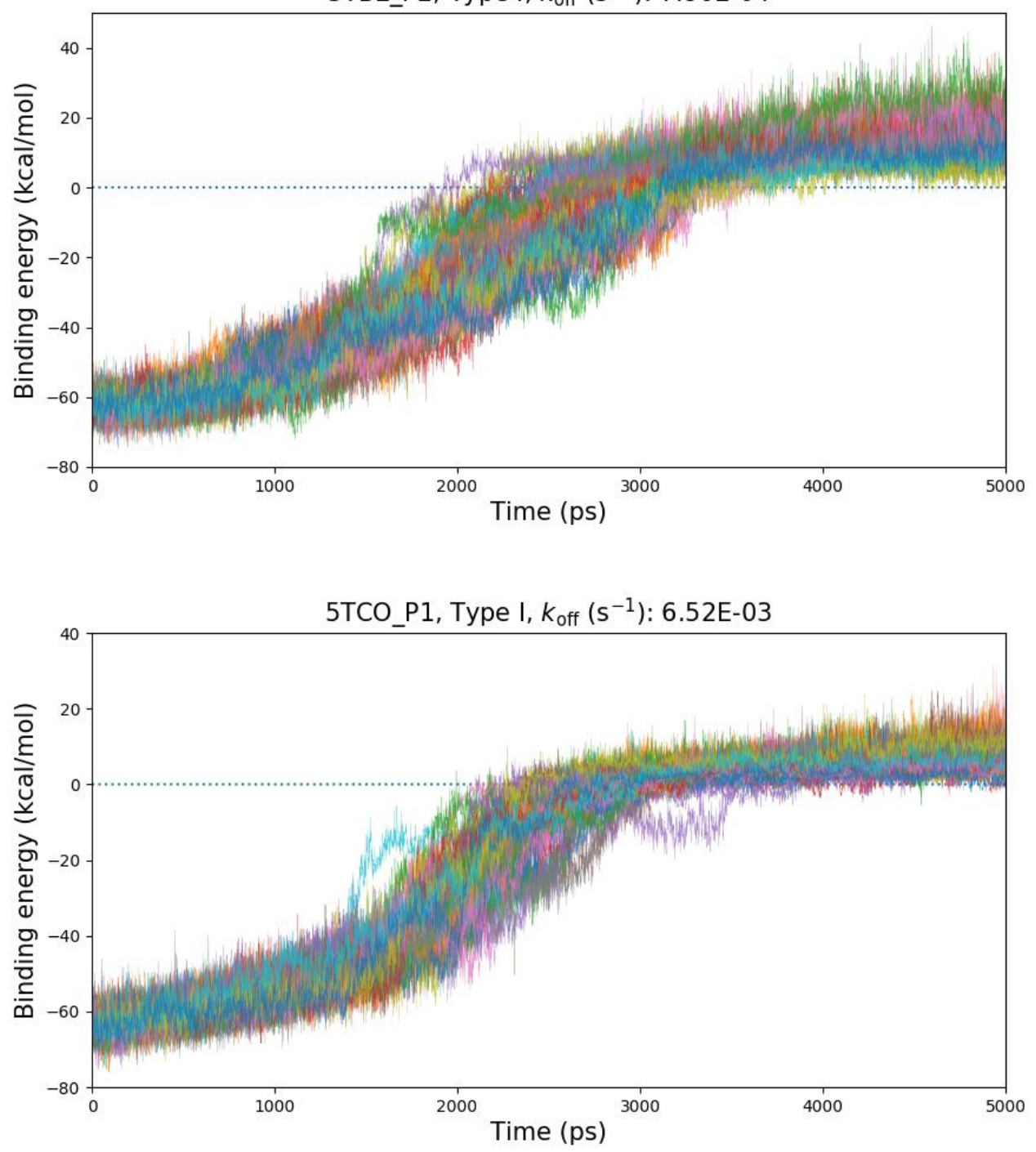


\section{Part 4. Local-scaled MD simulation with a finer scaling protocol}

We have re-run the local-scaled MD simulation with a "milder" scaling protocol on several most significant outliers in the data set. For this purpose, we employed the StatsModels module (version 0.11.1) in Python to analyze the influence of each sample in the linear regression conducted on the entire data set. As result, three most significant outliers (3D83, 4KSP, and 5N87) were identified with absolute error (studentized residual) larger than 2 units (see Fig. S4a). In order to make a comparison, we also added a complex well handled by our method (i.e. 4BTM) to this trial (see Fig. S4b). Adding this complex also ensures that the selected four complexes include two Type I and two Type II inhibitors. In the new local-scaled MD simulations of the four selected complexes, the electrostatic potential and the attractive part of Lennard-Jones potential were reduced to zero by $1 \%$ per $100 \mathrm{ps}$ in the first $10 \mathrm{~ns}$, and then both potentials were kept zero for another $10 \mathrm{~ns}$, producing a MD trajectory in a total length of $20 \mathrm{~ns}$. Other parameters used in MD were the same as described in our manuscript. A total of 10 replicas with different configurations were run for each complex, and the average binding energy integrals were computed using the same method described in our manuscript.

According to the newly computed binding energy integrals, the four selected complexes were ranked in exactly the same order. This observation suggests that the finer scaling protocol, which of course consumes more CPU time, may not be necessary for further improving the predictive accuracy of our method. Therefore, we have not attempted to repeat this trial on the other complexes in the data set. 


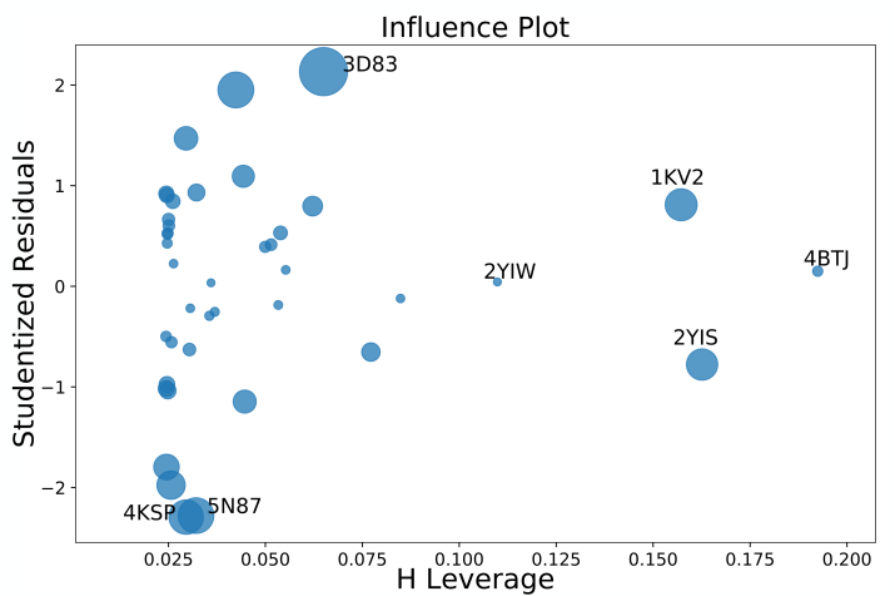

(a)

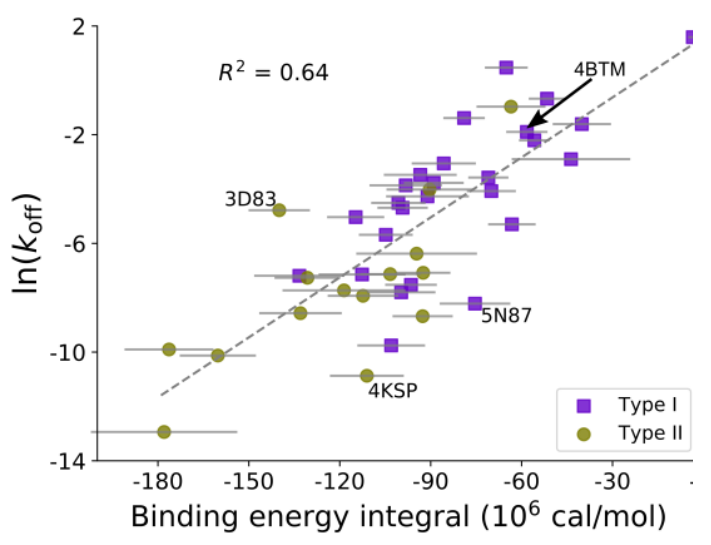

(b)

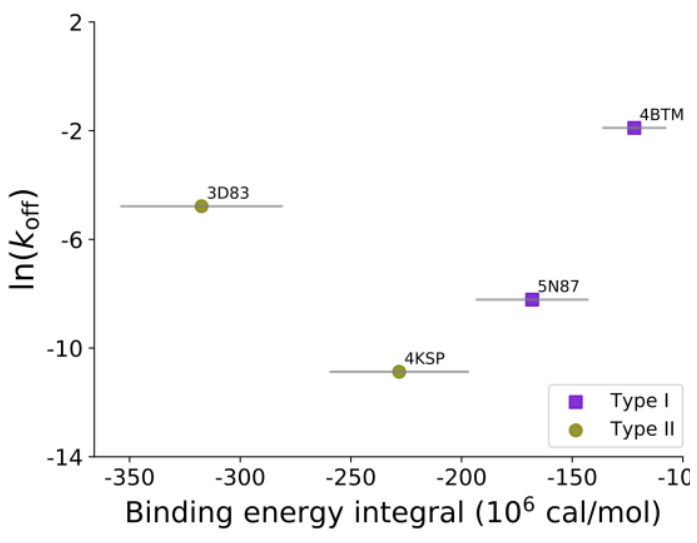

(c)

Figure S4. (a) Studentized residuals vs. the leverage of each complex in the data set. Three complexes with absolute error (studentized residuals) over 2.0 are considered to be the most significant outliers. (b) Correlation between $\ln (\mathrm{koff})$ values and the binding energy integrals given by our method on the whole data set, where the three selected outliers (3D83, 4KSP, 5N87) and a well-handled sample (4BTM) are labelled. (c) The binding energy integrals computed by the finer scaling protocol for four selected complexes. For each sample, the error bar indicates the standard deviation in the computed binding energy integral. 


\section{Part 5. Ligand dissociation path of each complex}

Figure S5. Hinge-Glu and DFG-Phe were projected on the $Y-Z$ plane. HingeGlu's locations in Type I are shown in blue; Type II in red. DFG-Phe's locations in Type I are shown in dark yellow; Type II in yellow. Black points represent the locations of Hinge-Glu and DFG-Phe in current complex. The exit paths of each complex were projected on the $\mathrm{Y}-\mathrm{Z}$ plane. Different clusters were represented by different colors. The numbers of trajectory are showed at the end of lines.

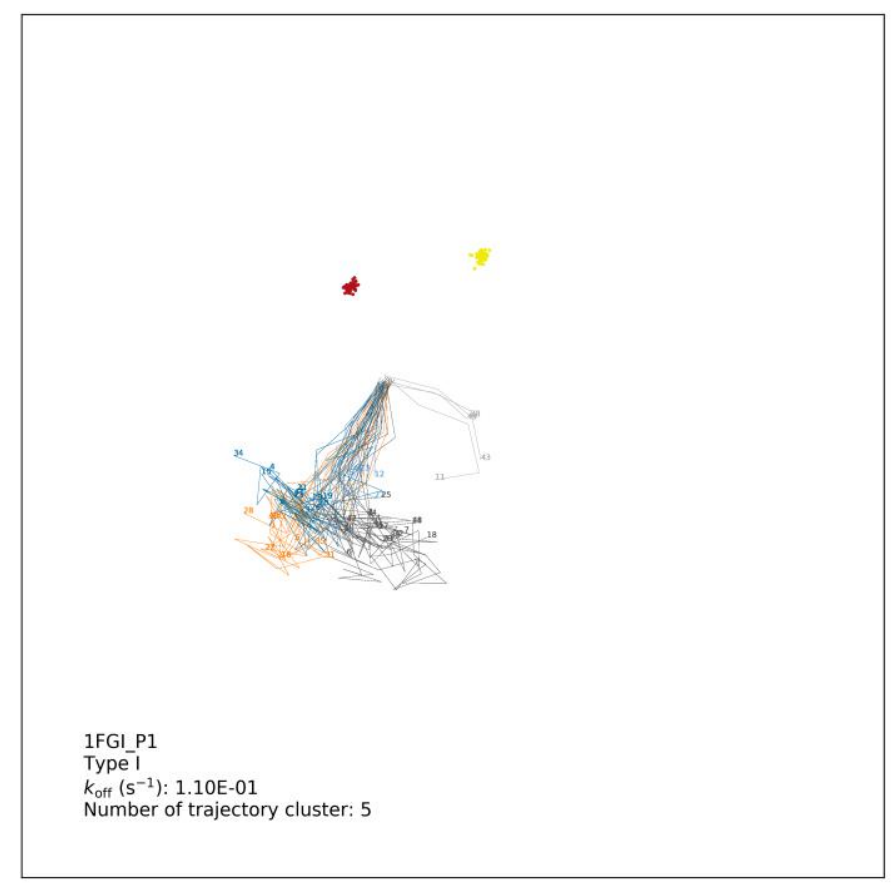



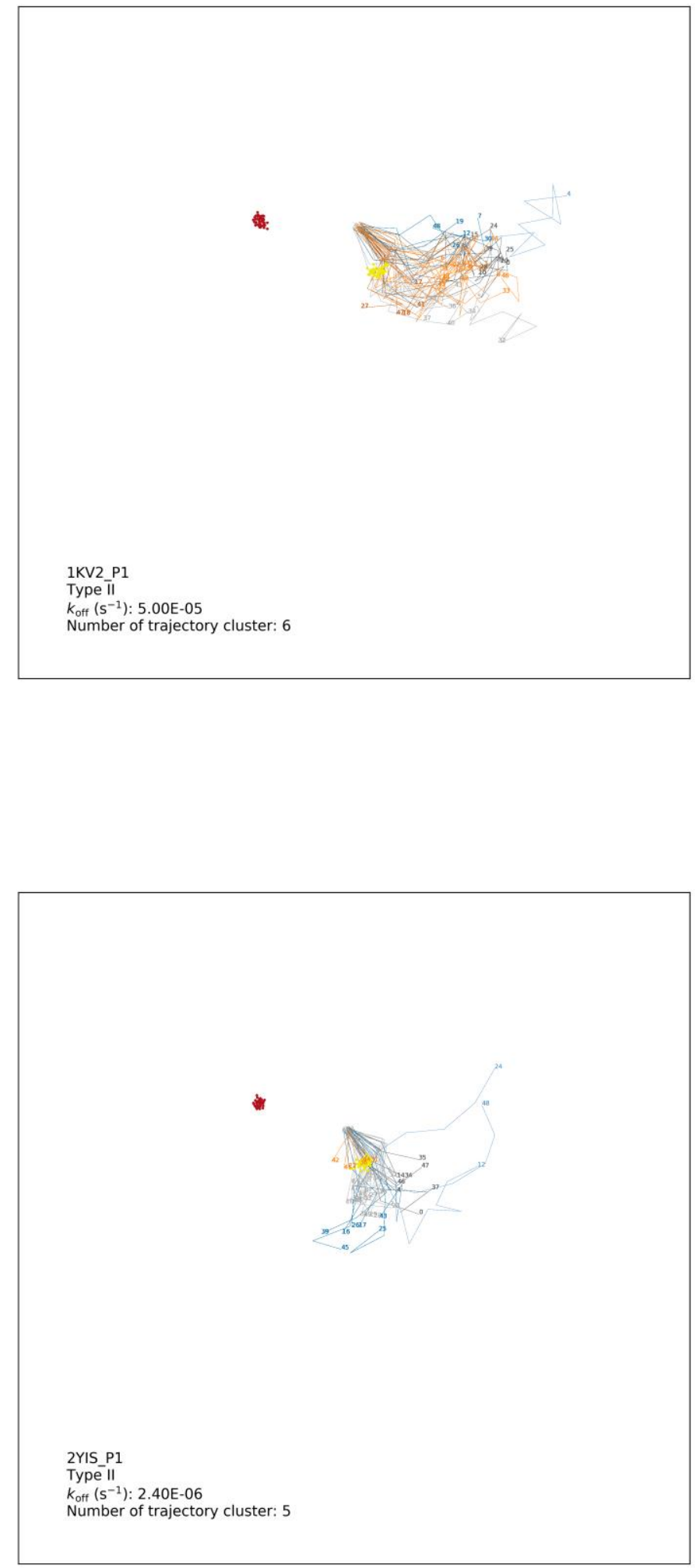

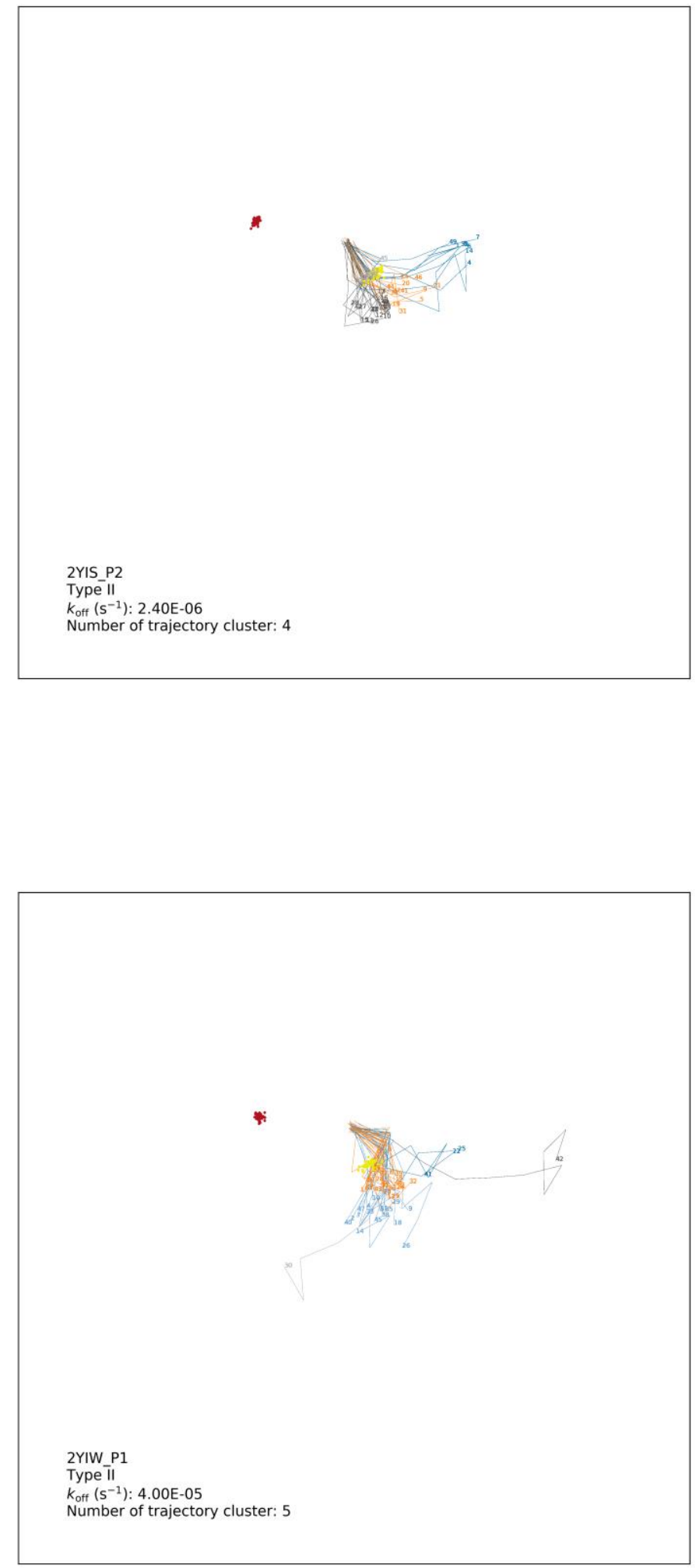

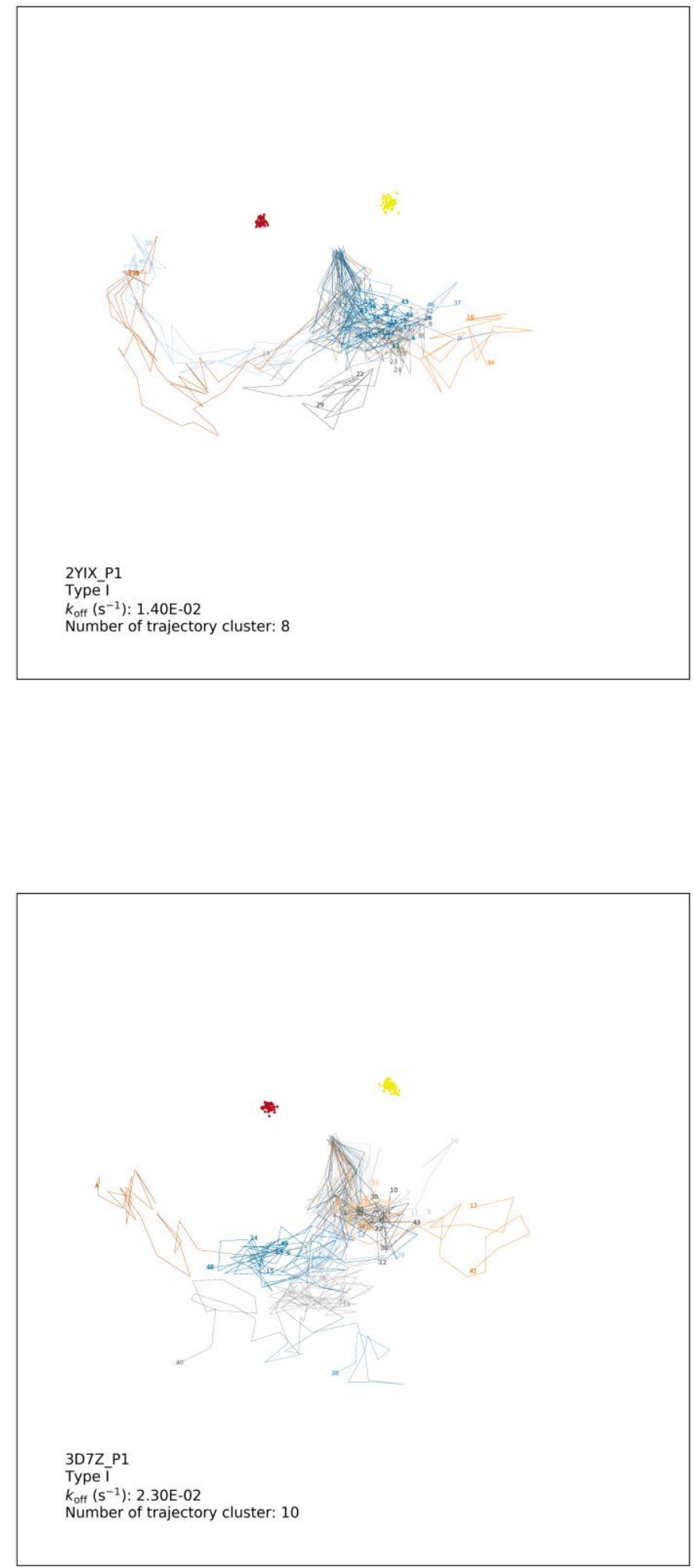

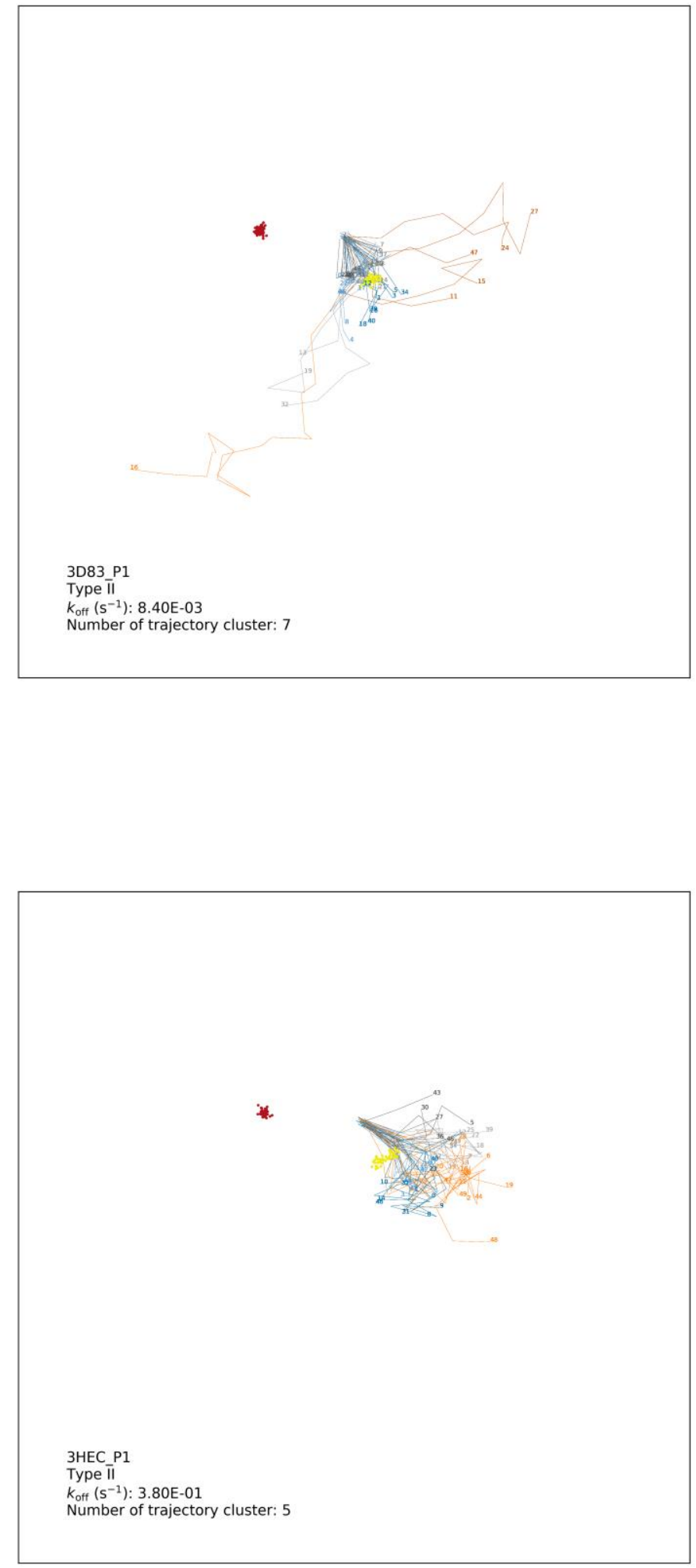

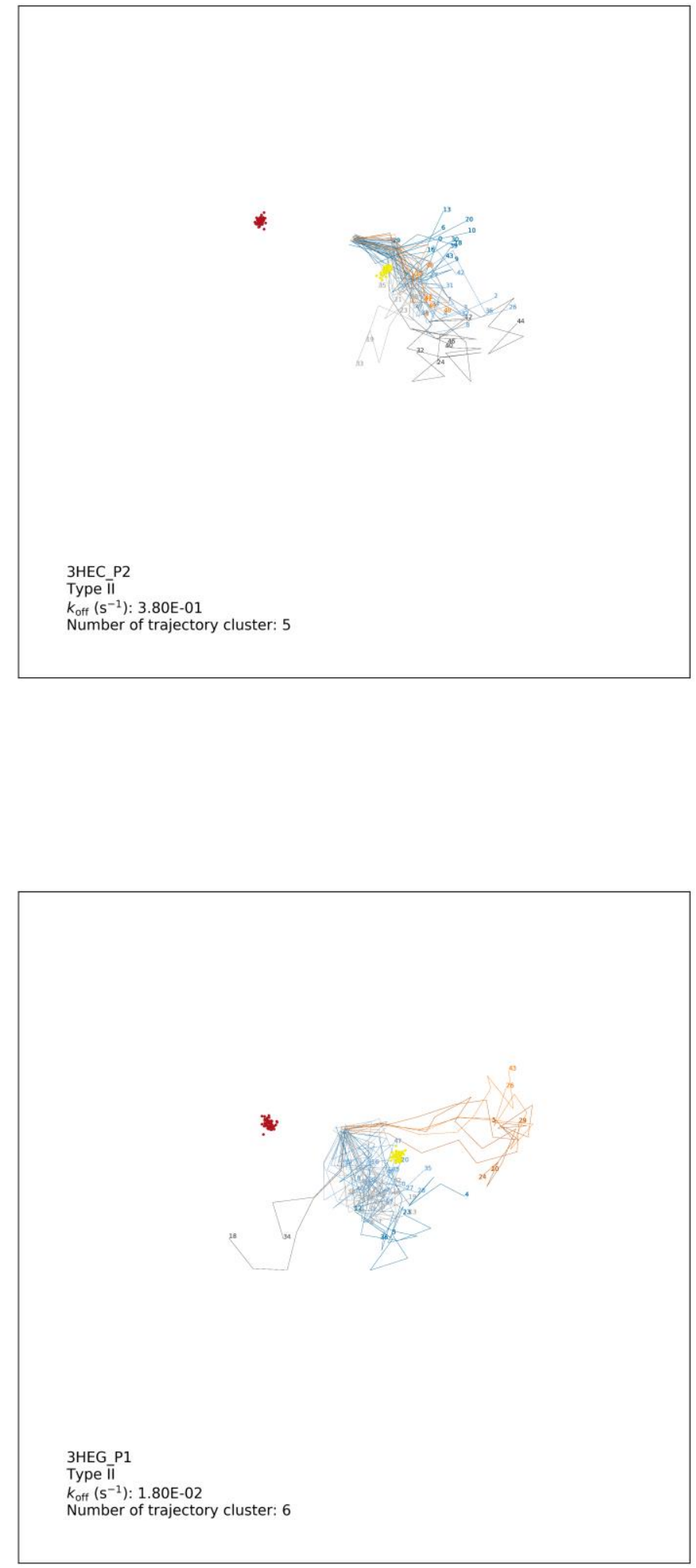

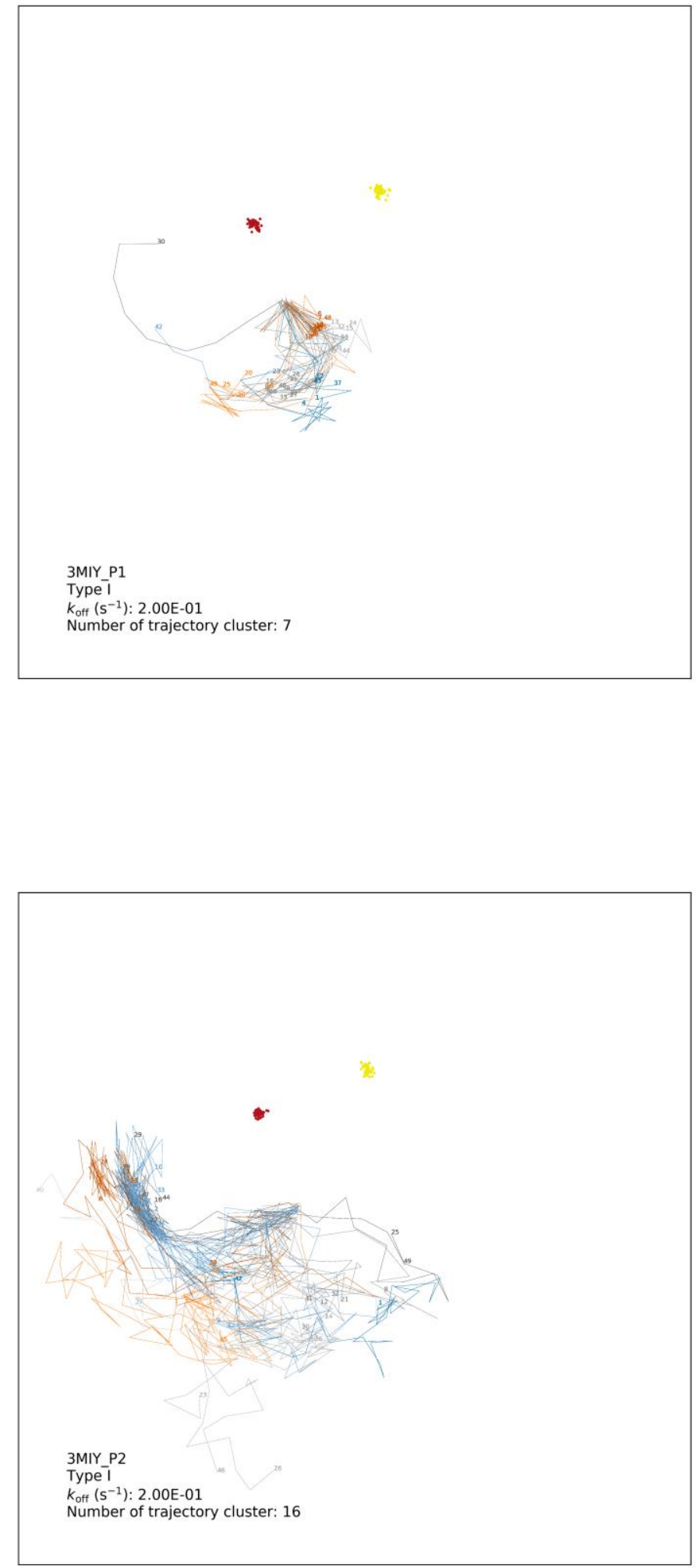

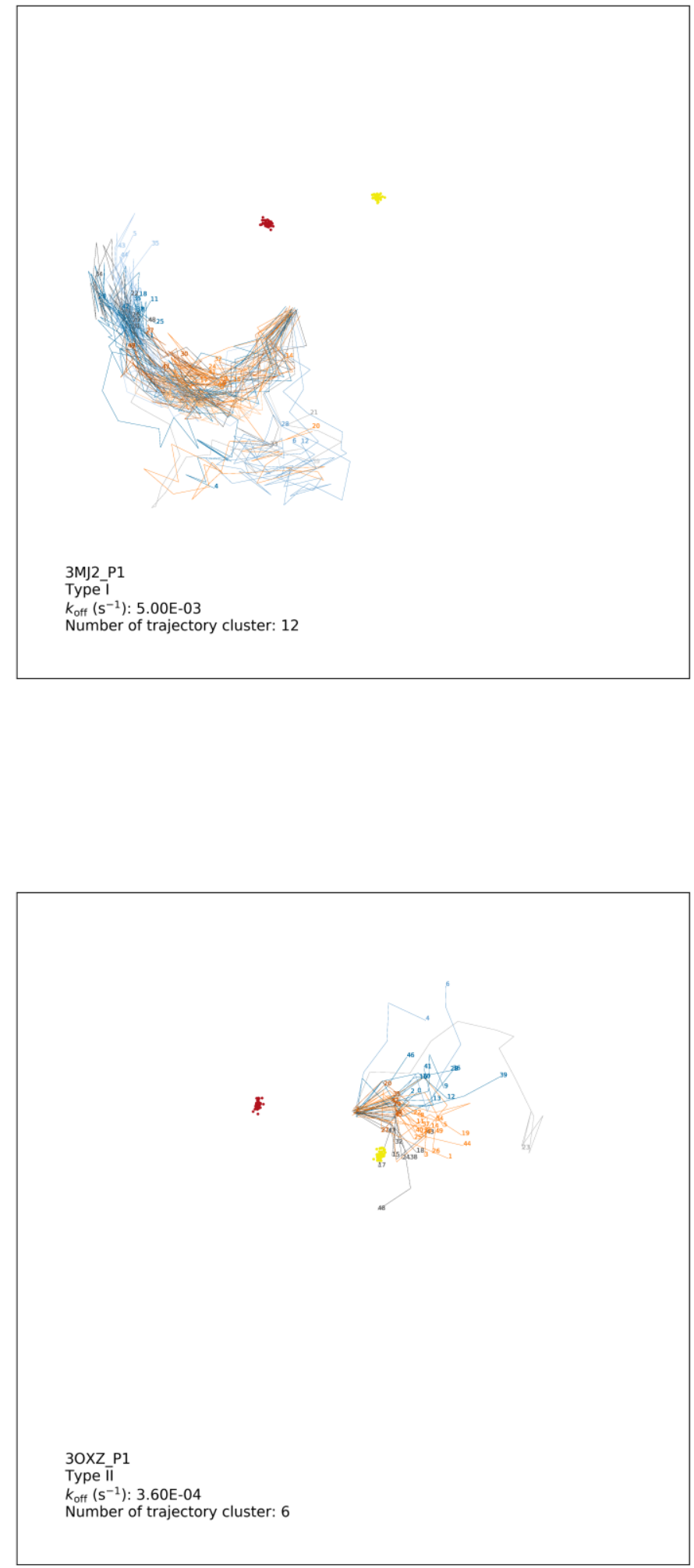

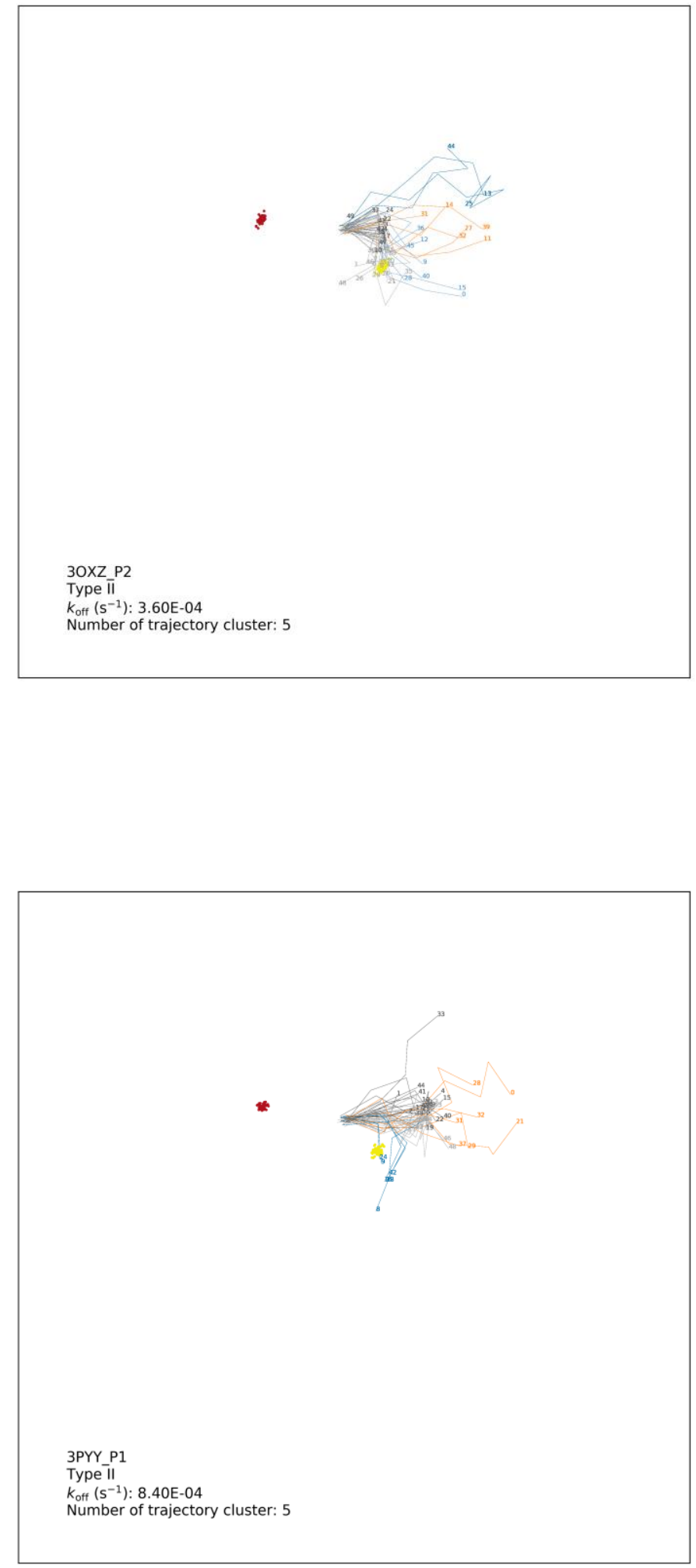

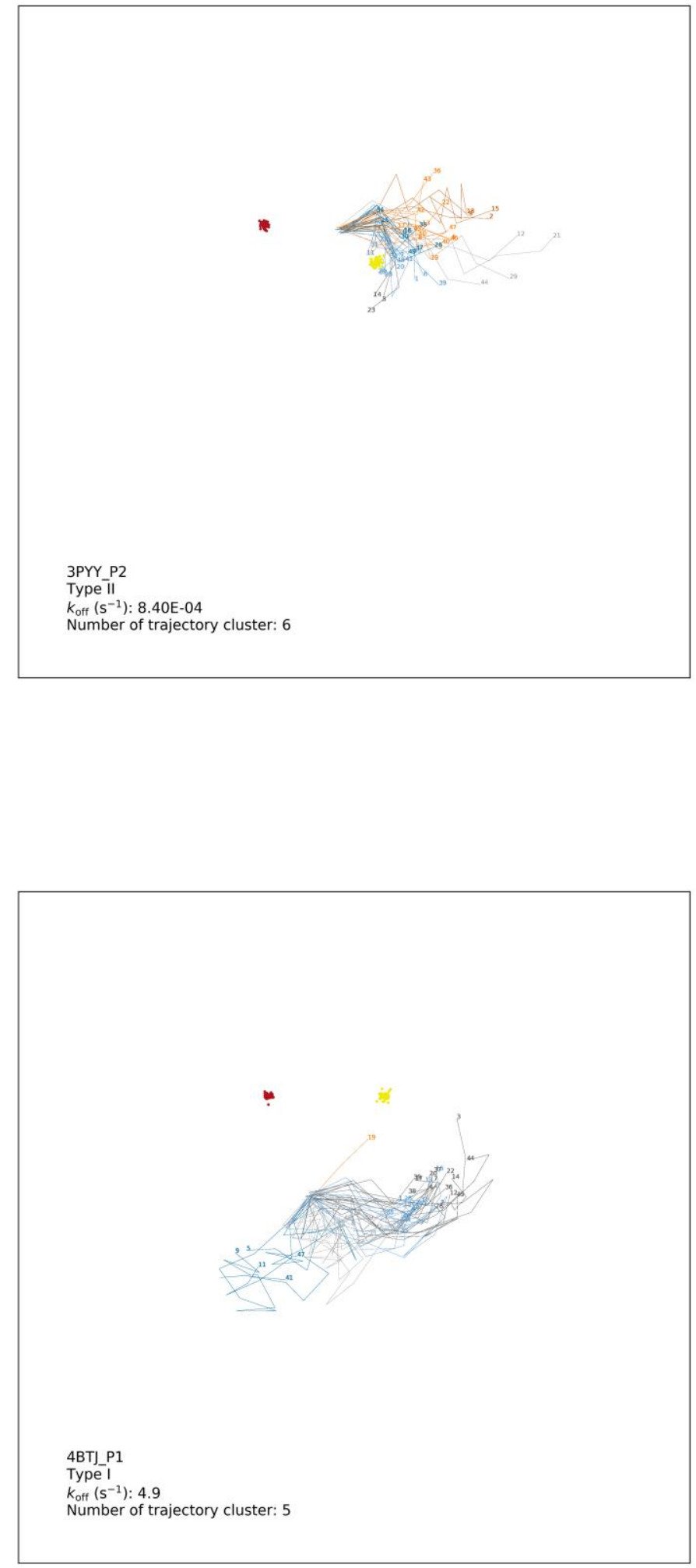

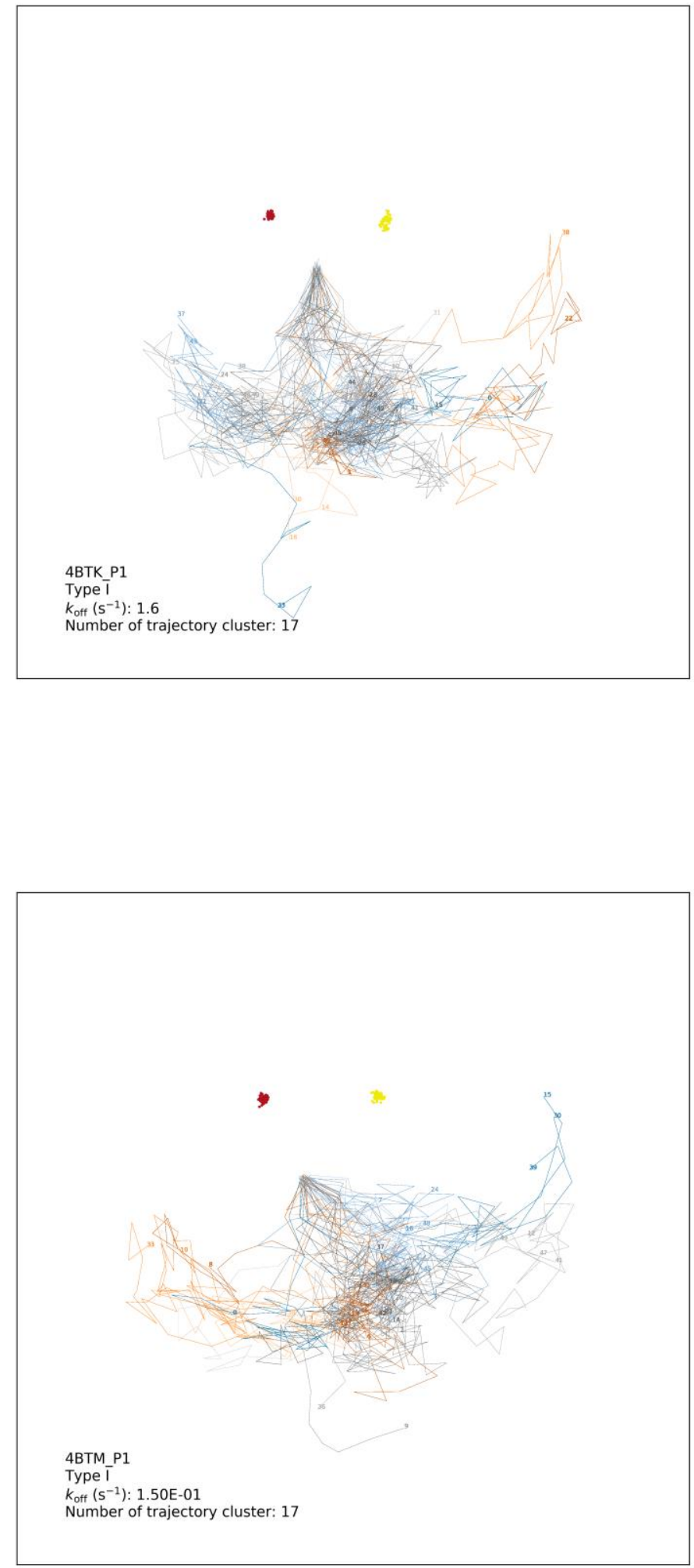

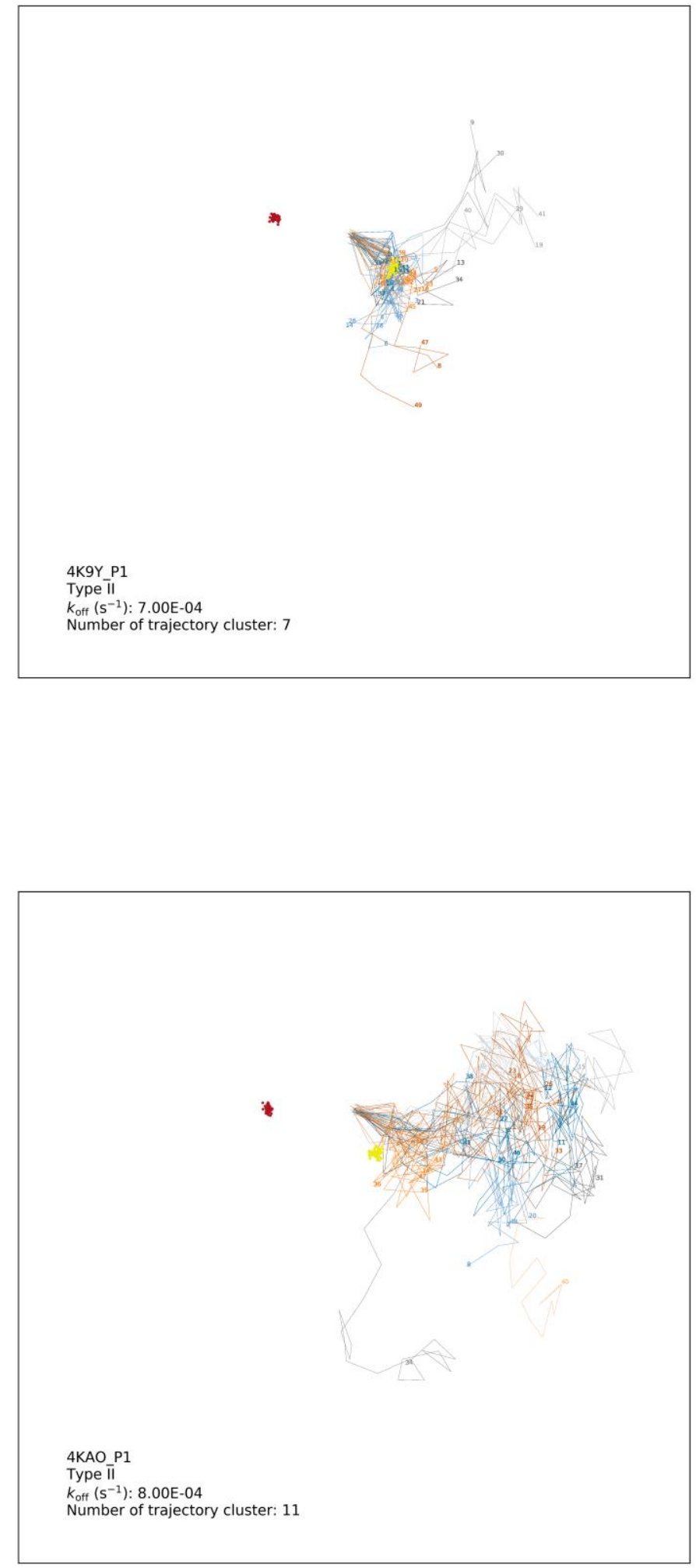

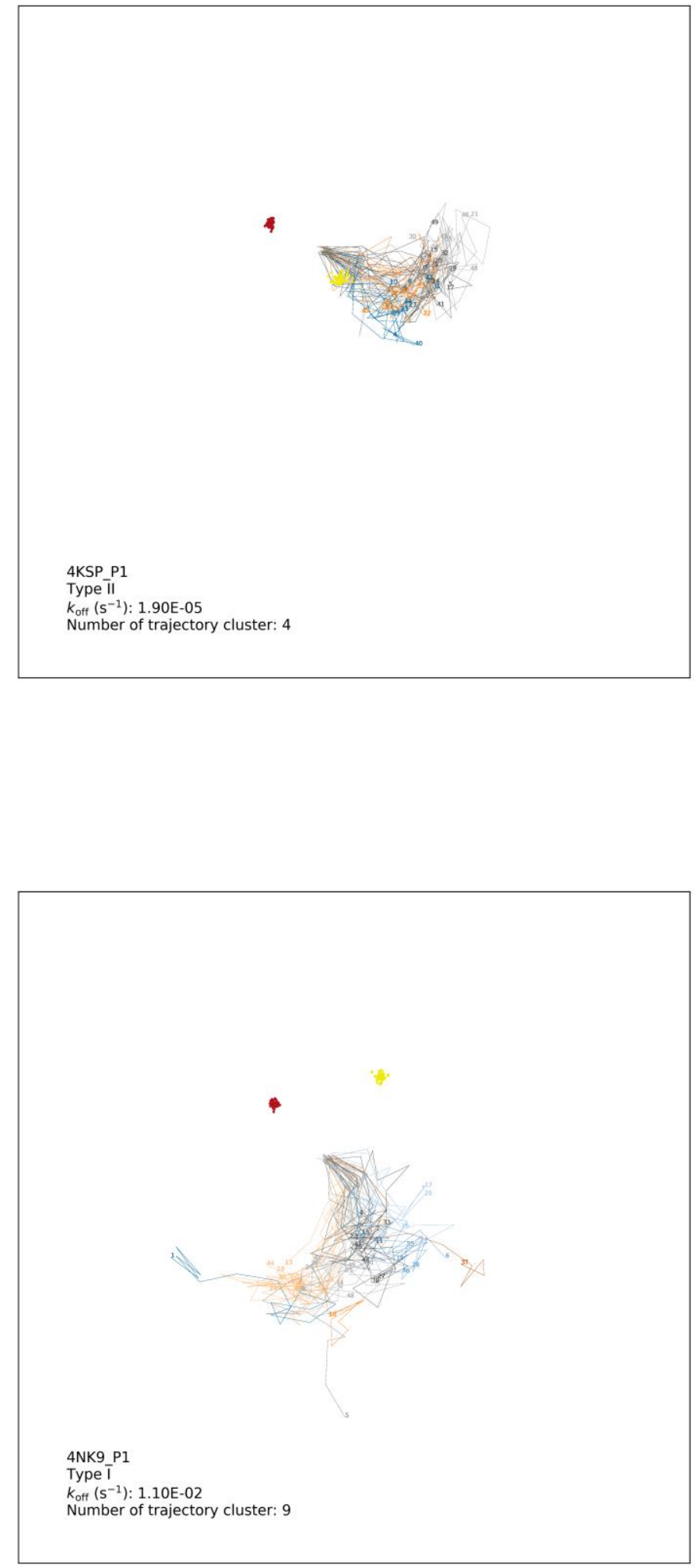

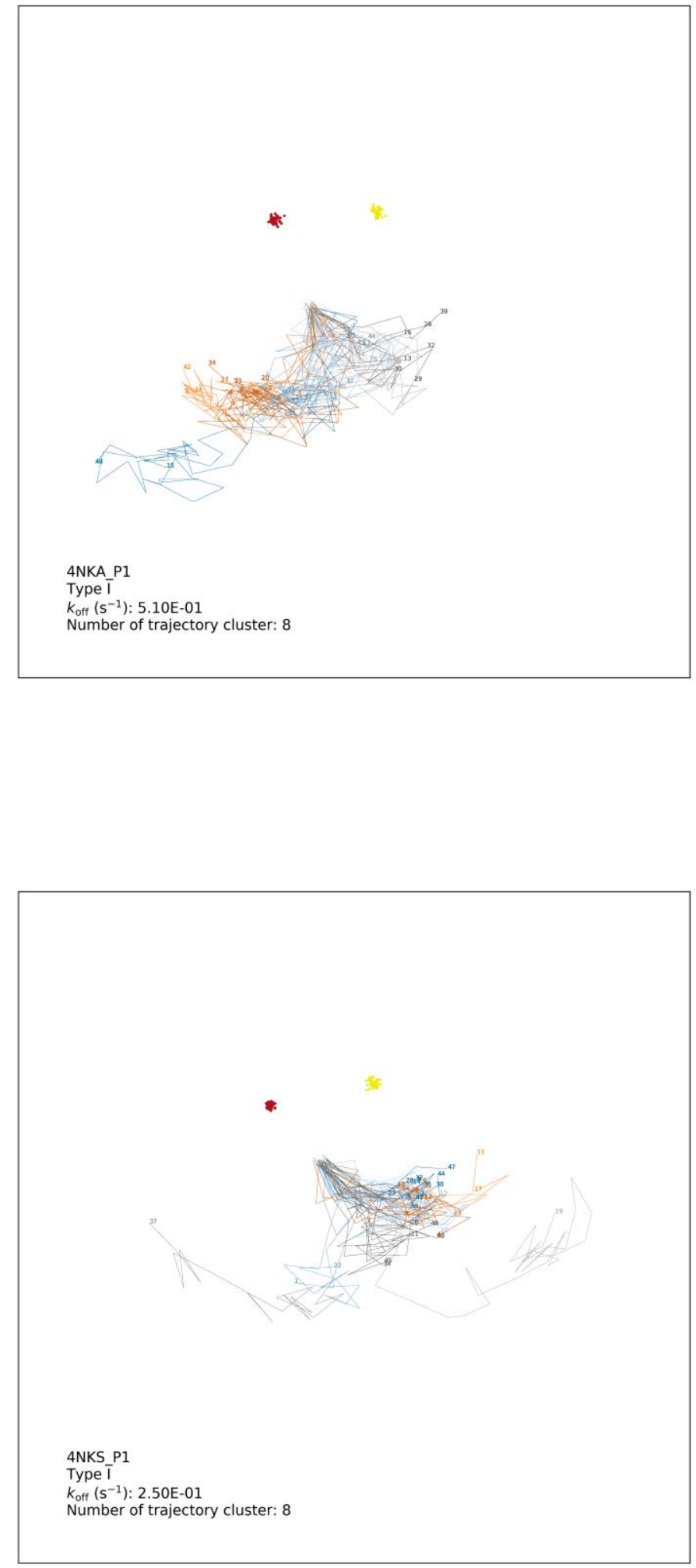

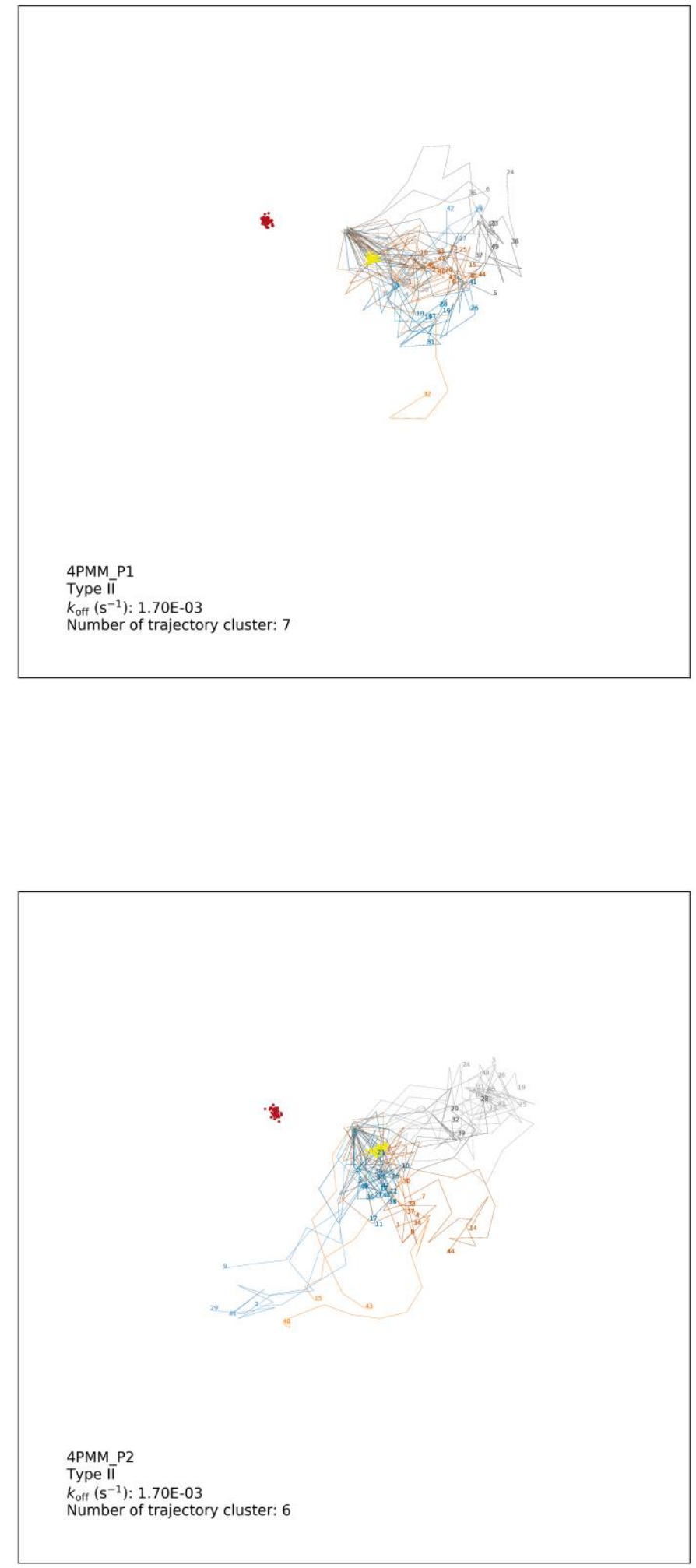

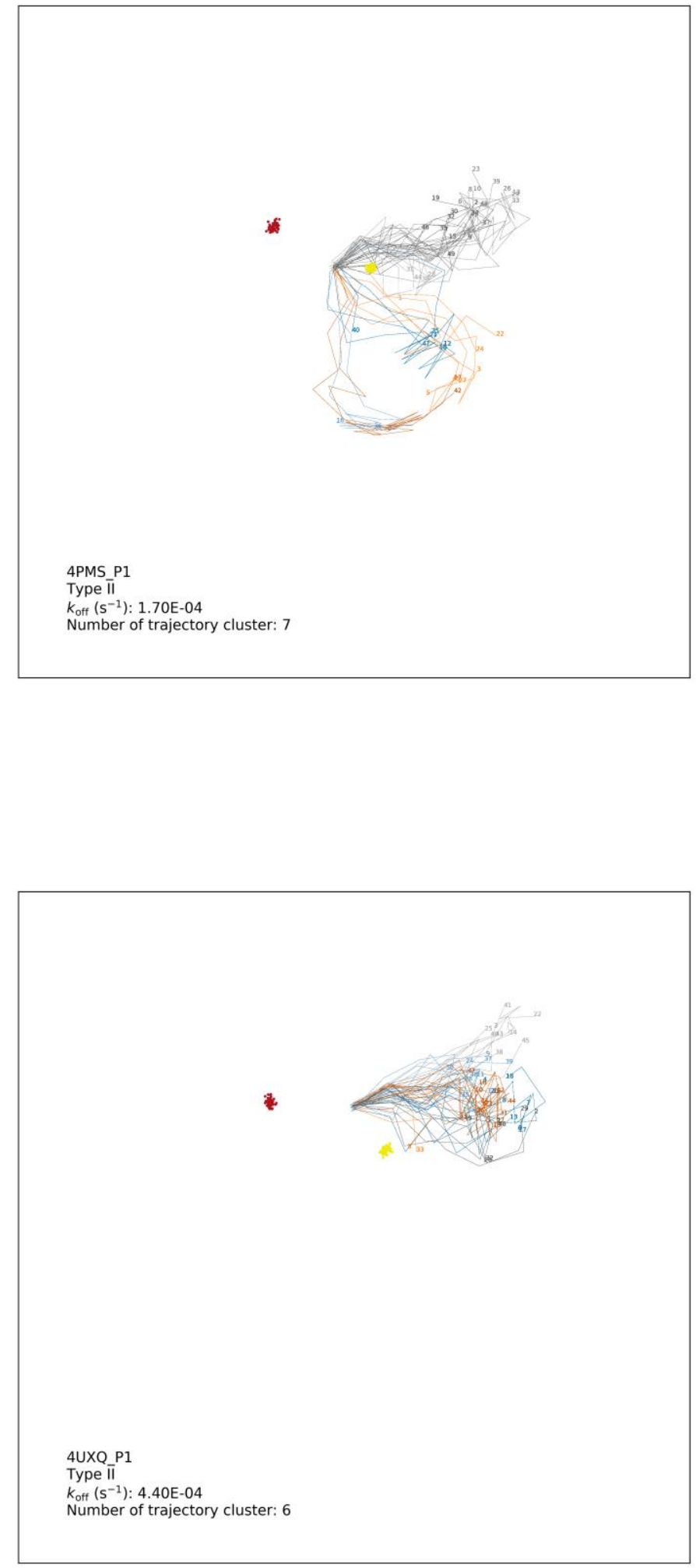

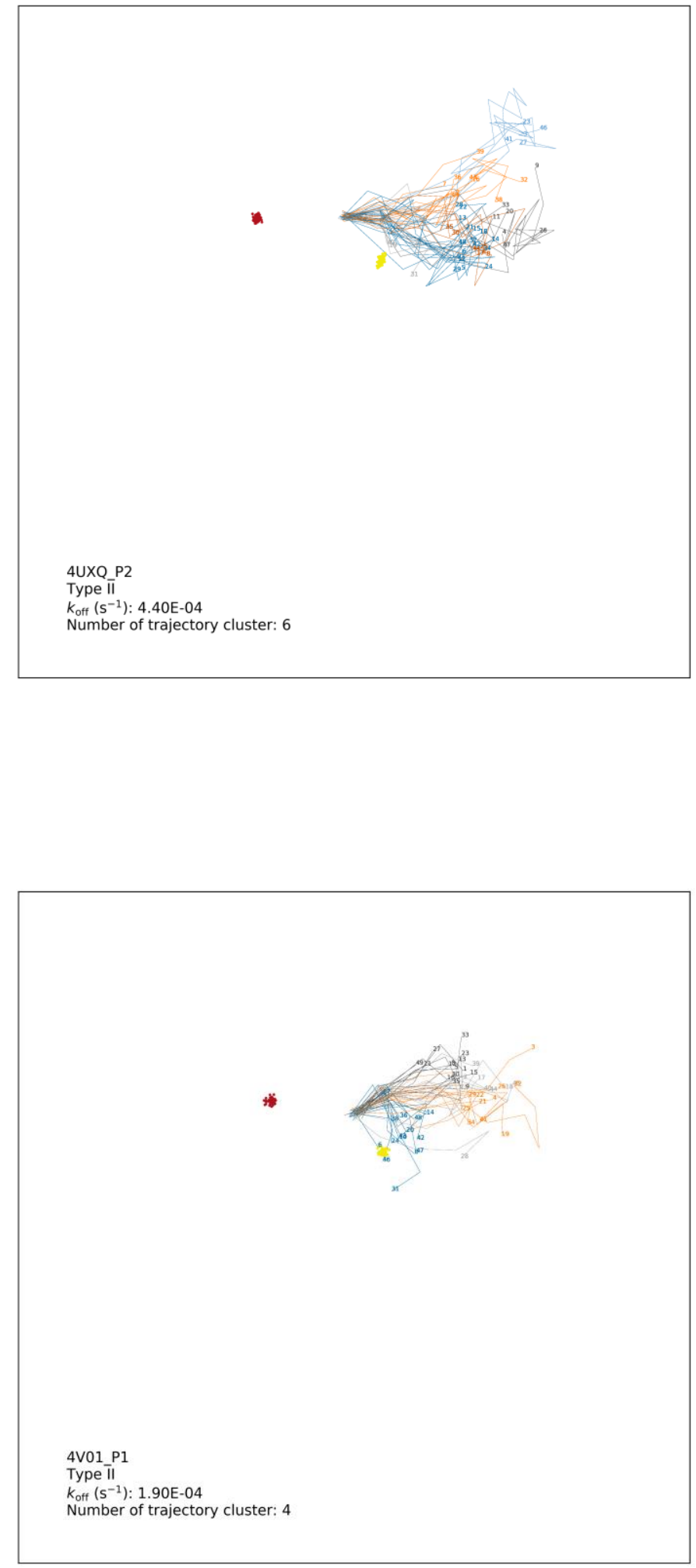

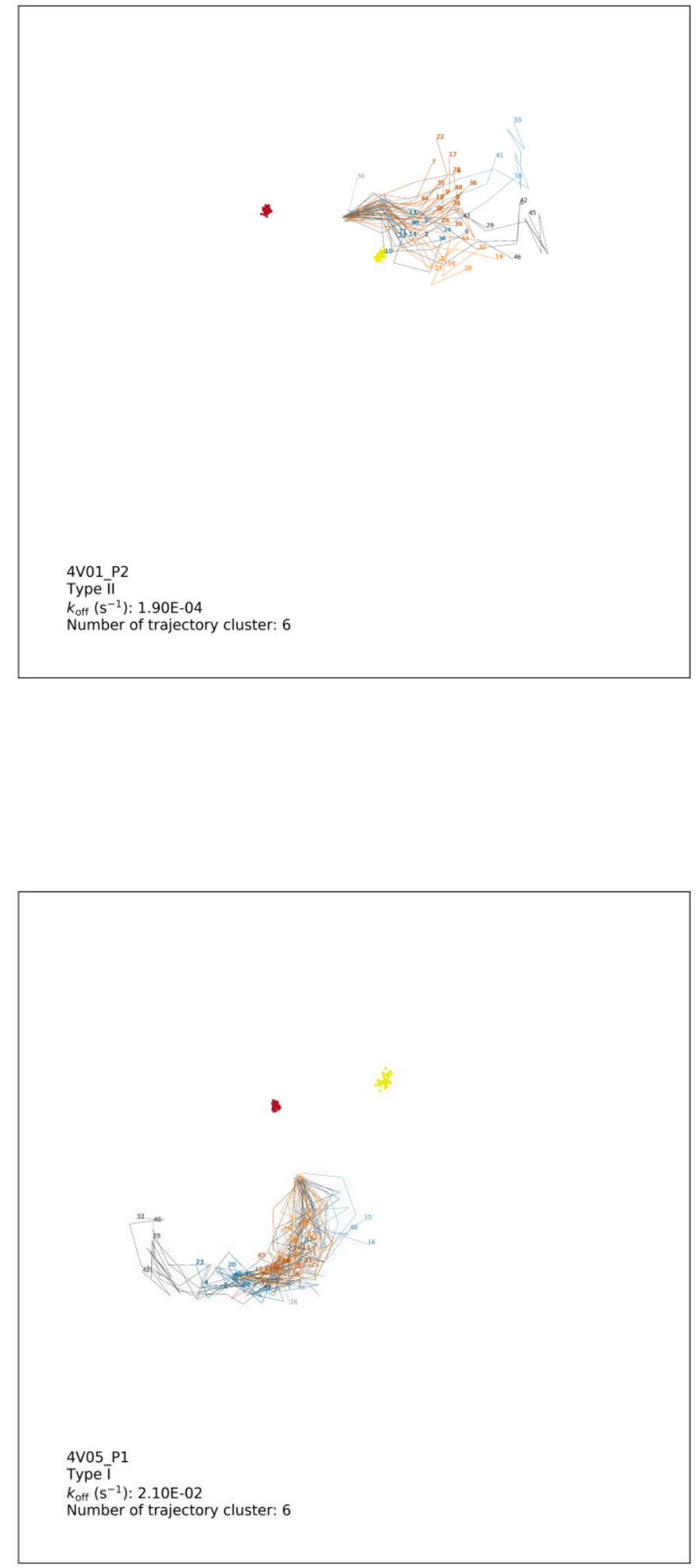

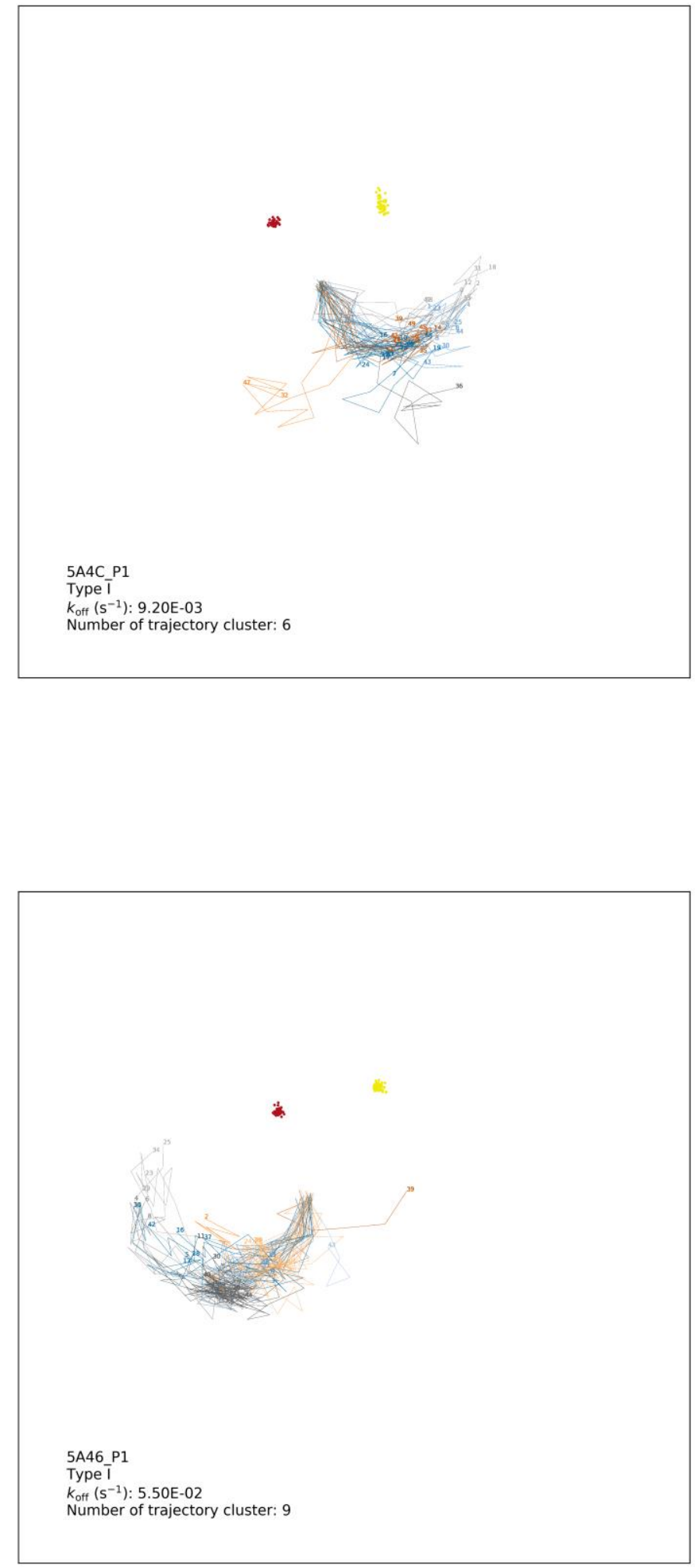

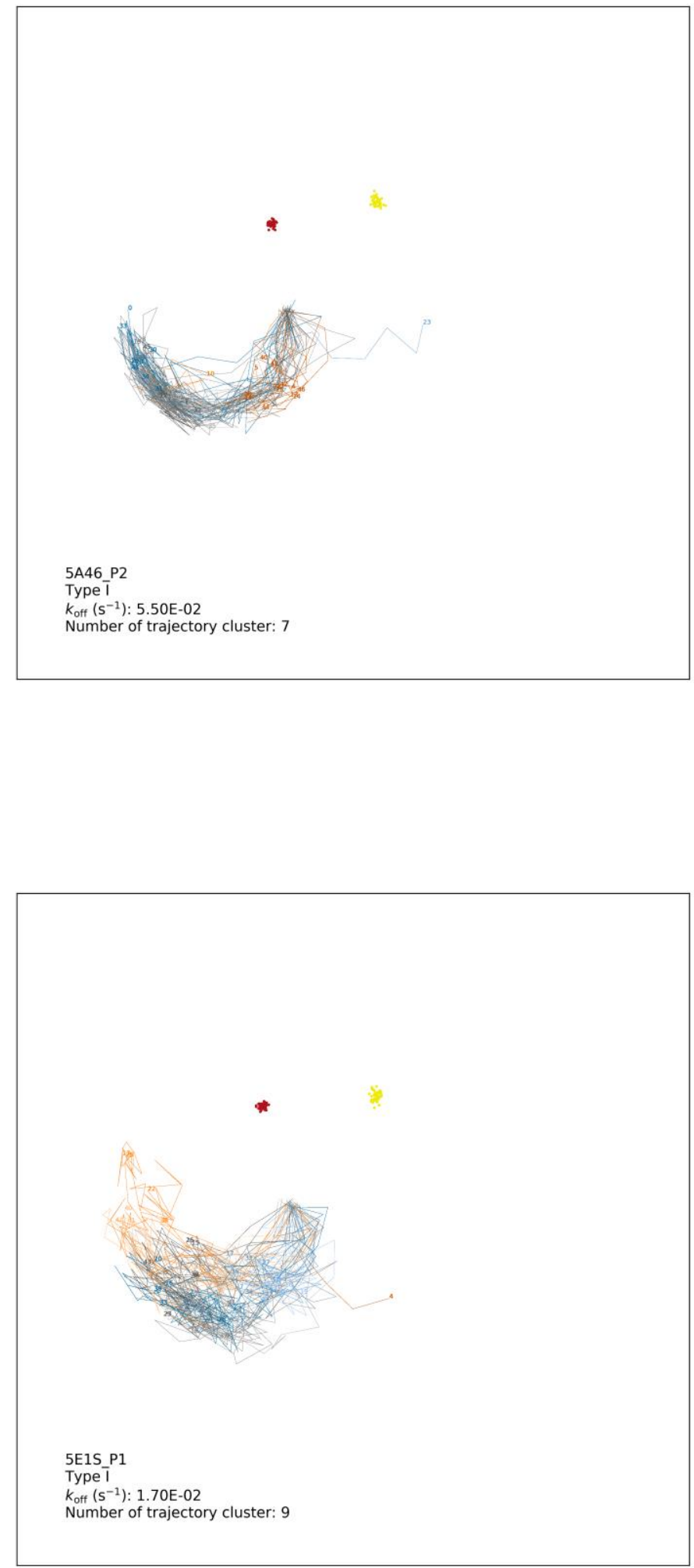

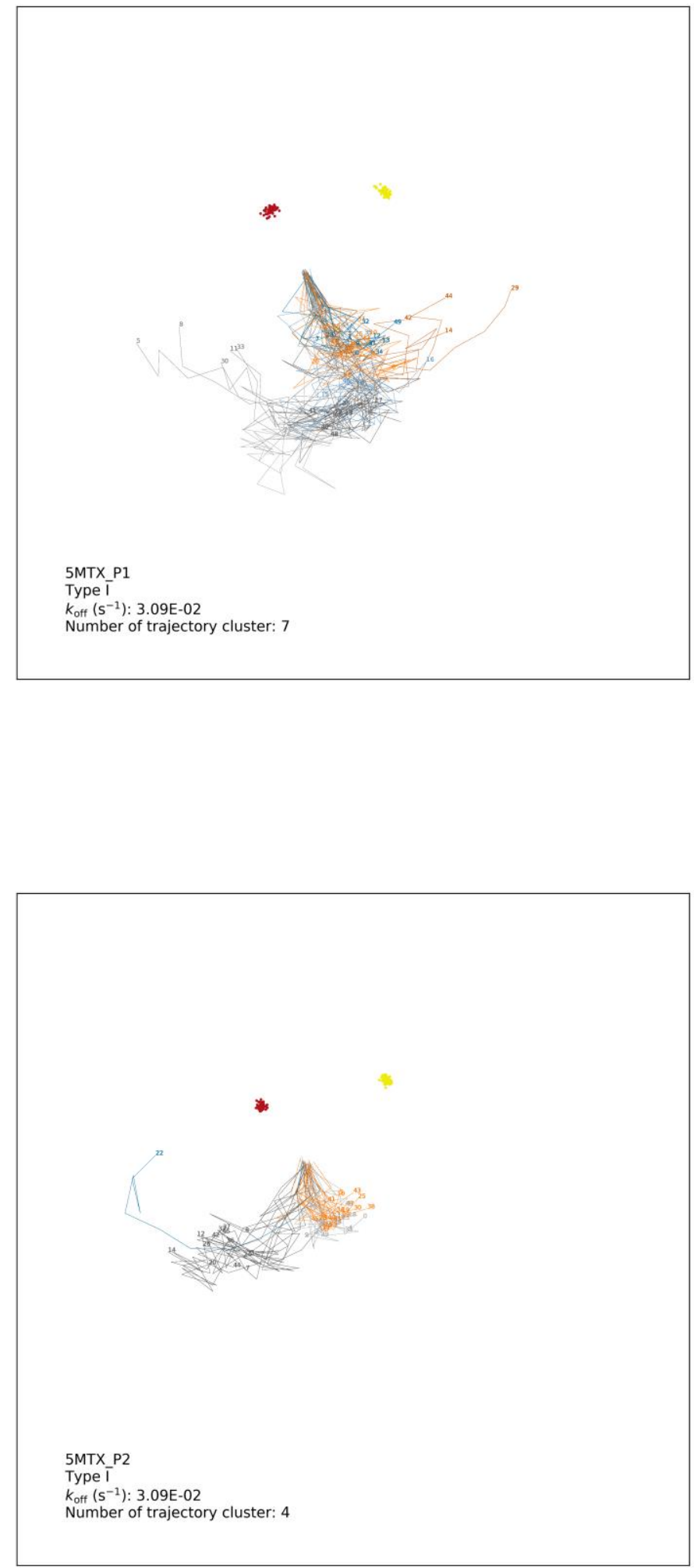

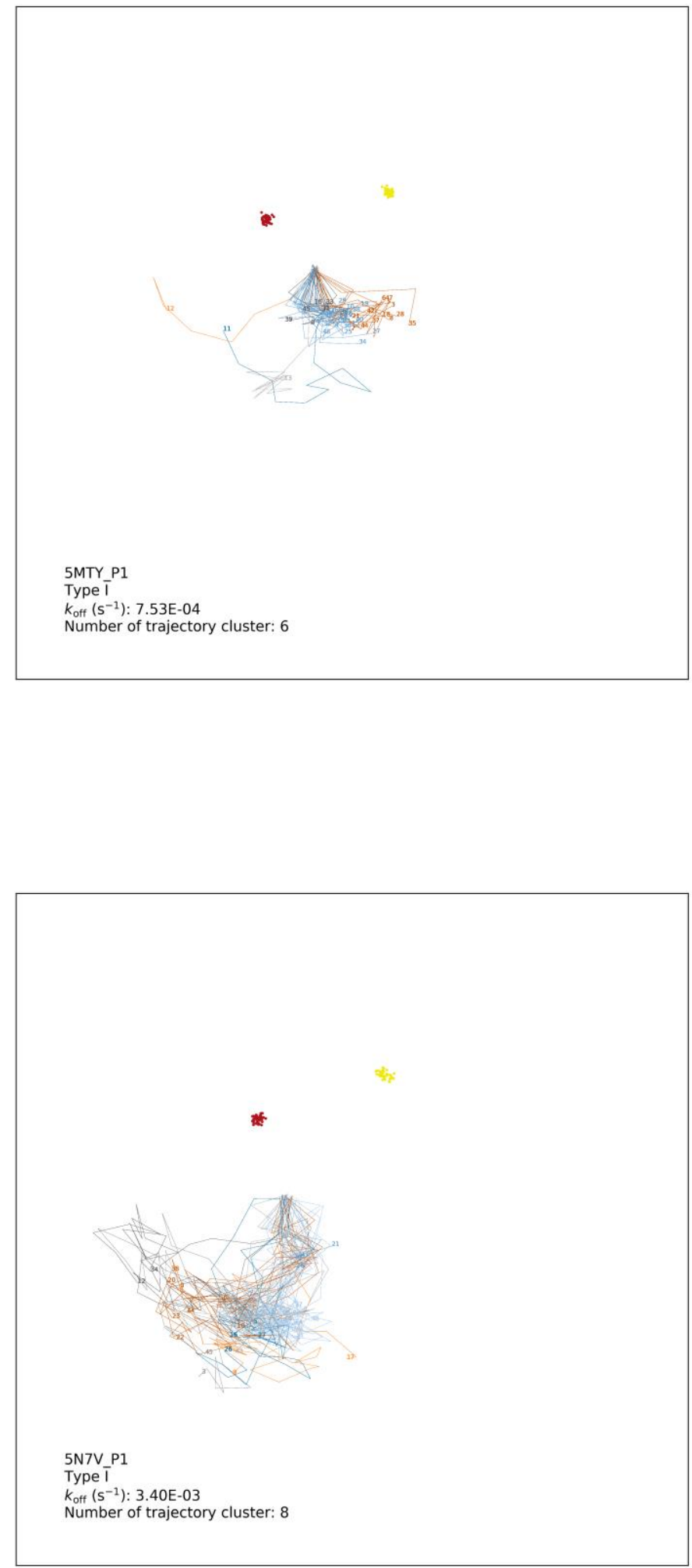

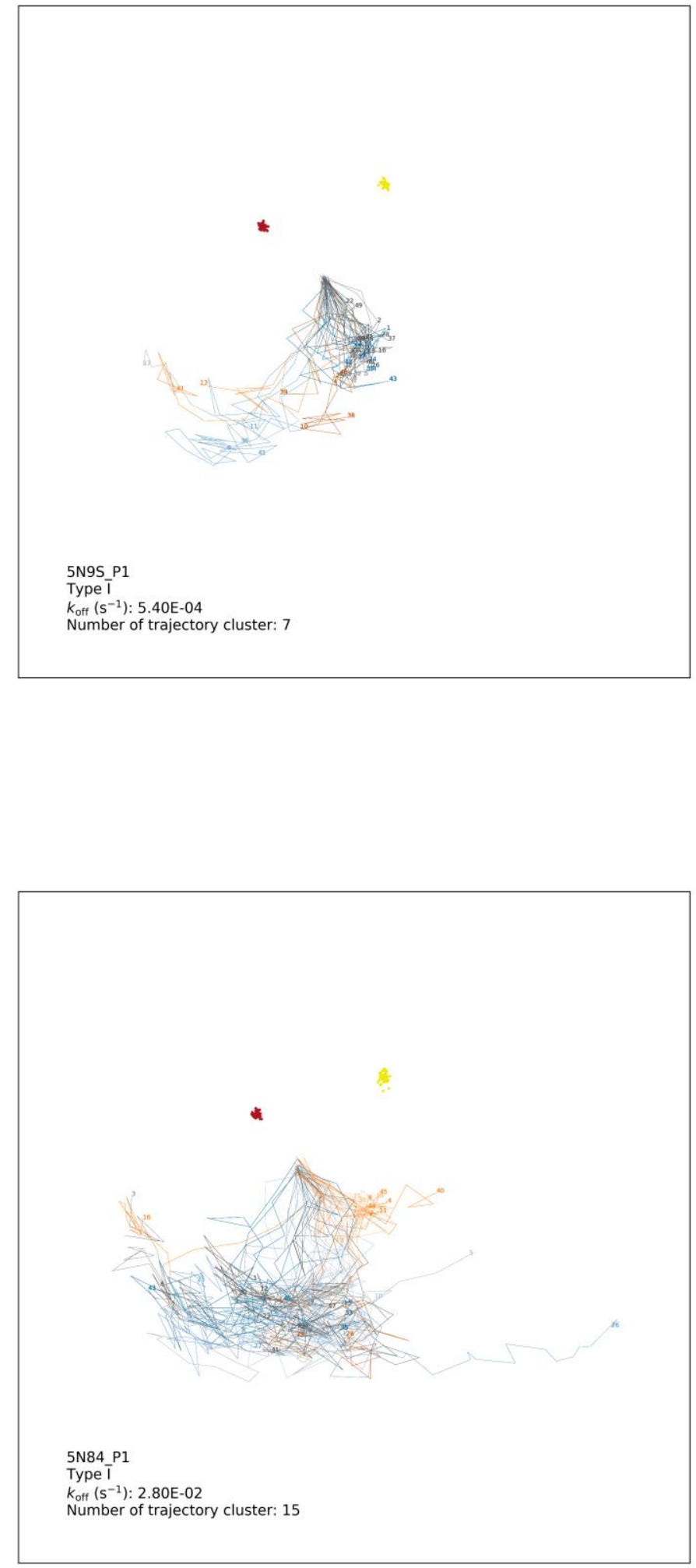

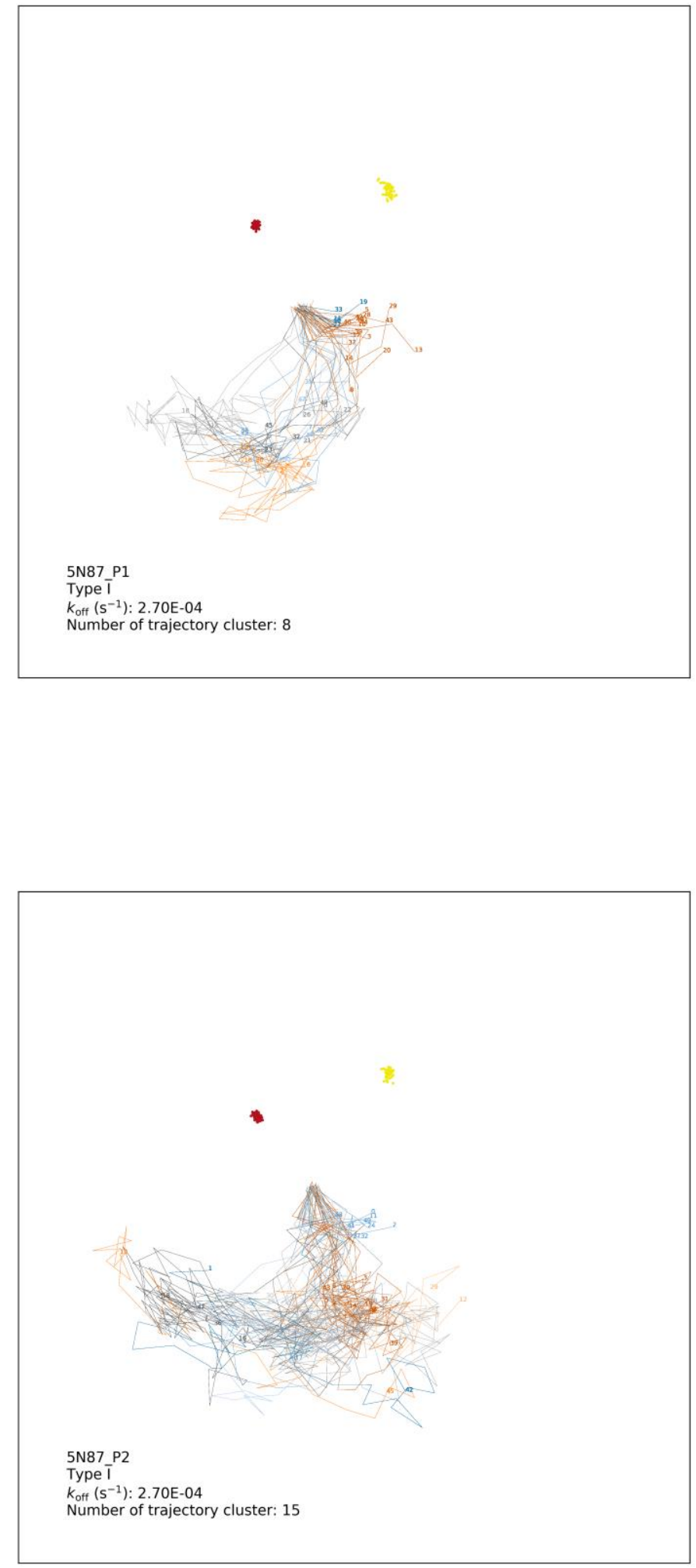

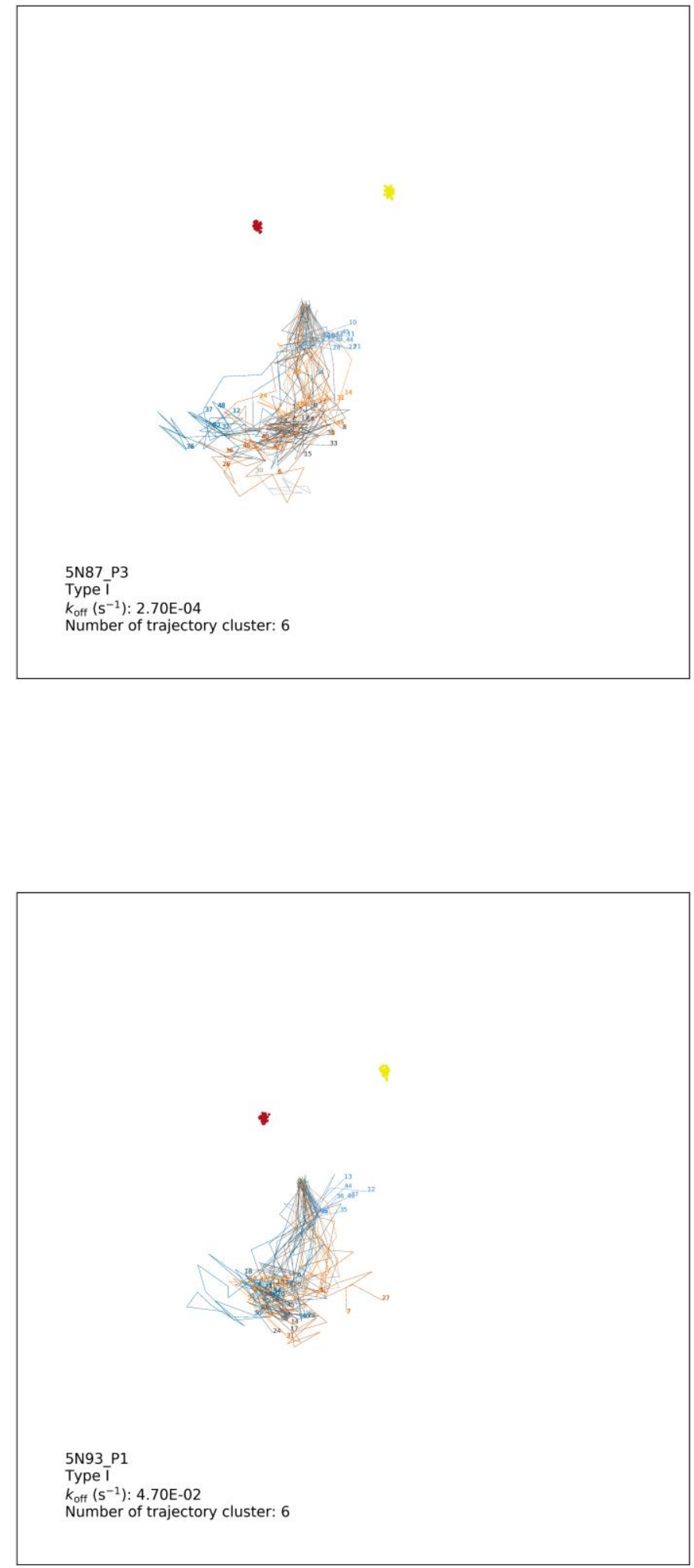

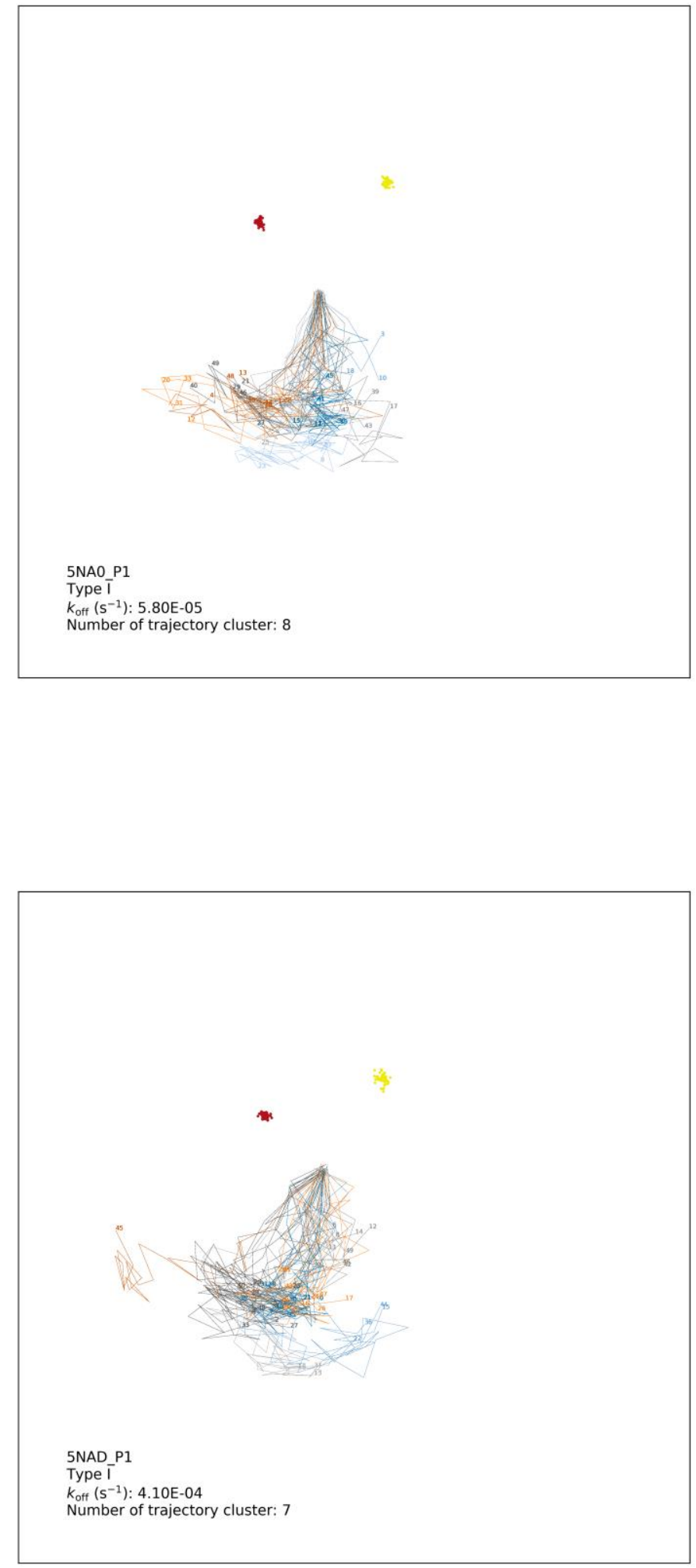

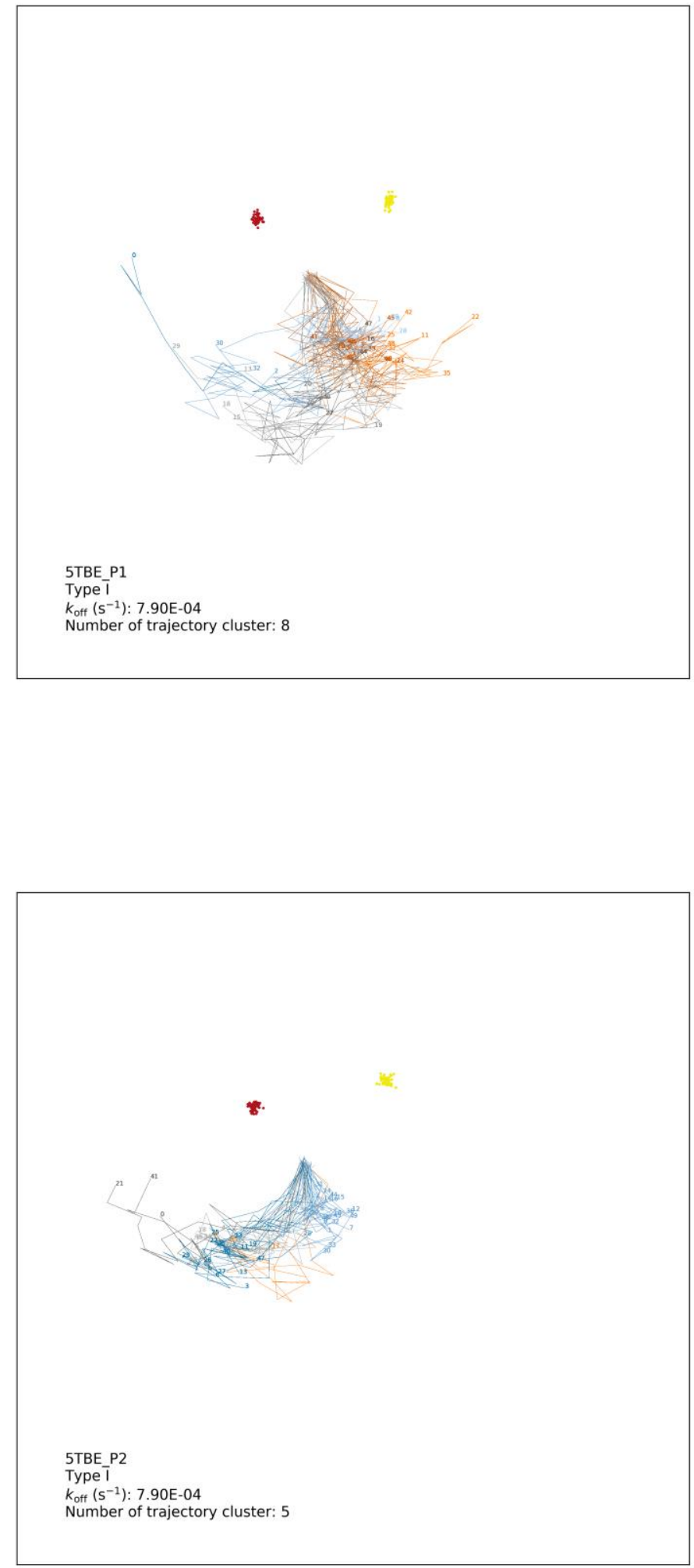


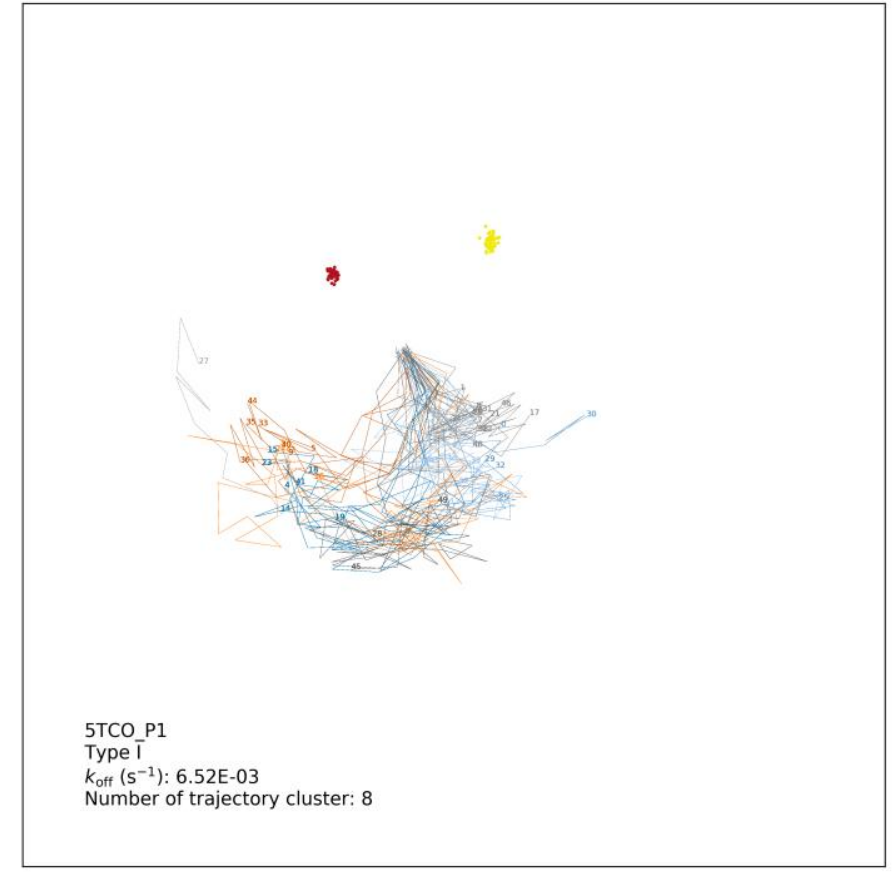




\section{Part 6. Conformational landscapes of dynamic and static}

\section{protein structures}
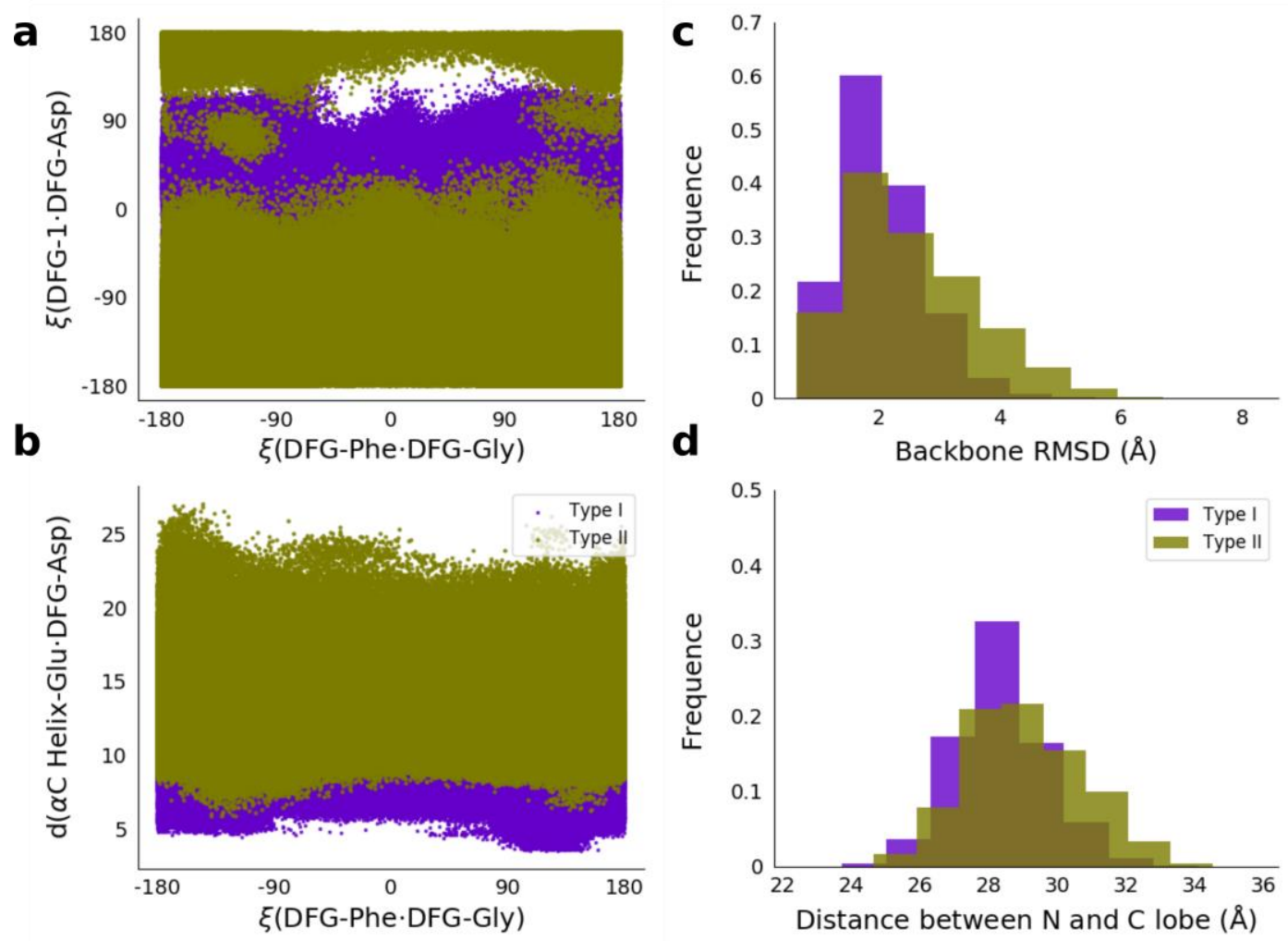

Figure S6. The protein conformational landscape of the whole dissociation

process. $(a, b)$ Each square and circle represents a conformation from localscaled MD. The purple and brown represent the Type I and Type II inhibitorsbound kinases. The $\xi$ is the pseudodihedral angle of four consecutive alphacarbon atoms. DFG-Phe.DFG-Gly is the short form of DFG-Asp, DFG-Phe, DFGGly, and DFG+1 residues; DFG-1.DFG-Asp is DFG-2, DFG-1, DFG-Asp, and DFG-Phe residues. $d(\alpha C$ Helix-Glu-DFG-Asp) is the distance between the alphacarbon atoms of $\alpha$ C Helix-Glu and DFG-Asp residues. (c, d) The distribution of the backbone RMSD and distance between N-lobe and C-lobe of Type I and Type II inhibitors-bound kinases. The color is the same as $(a, b)$. 

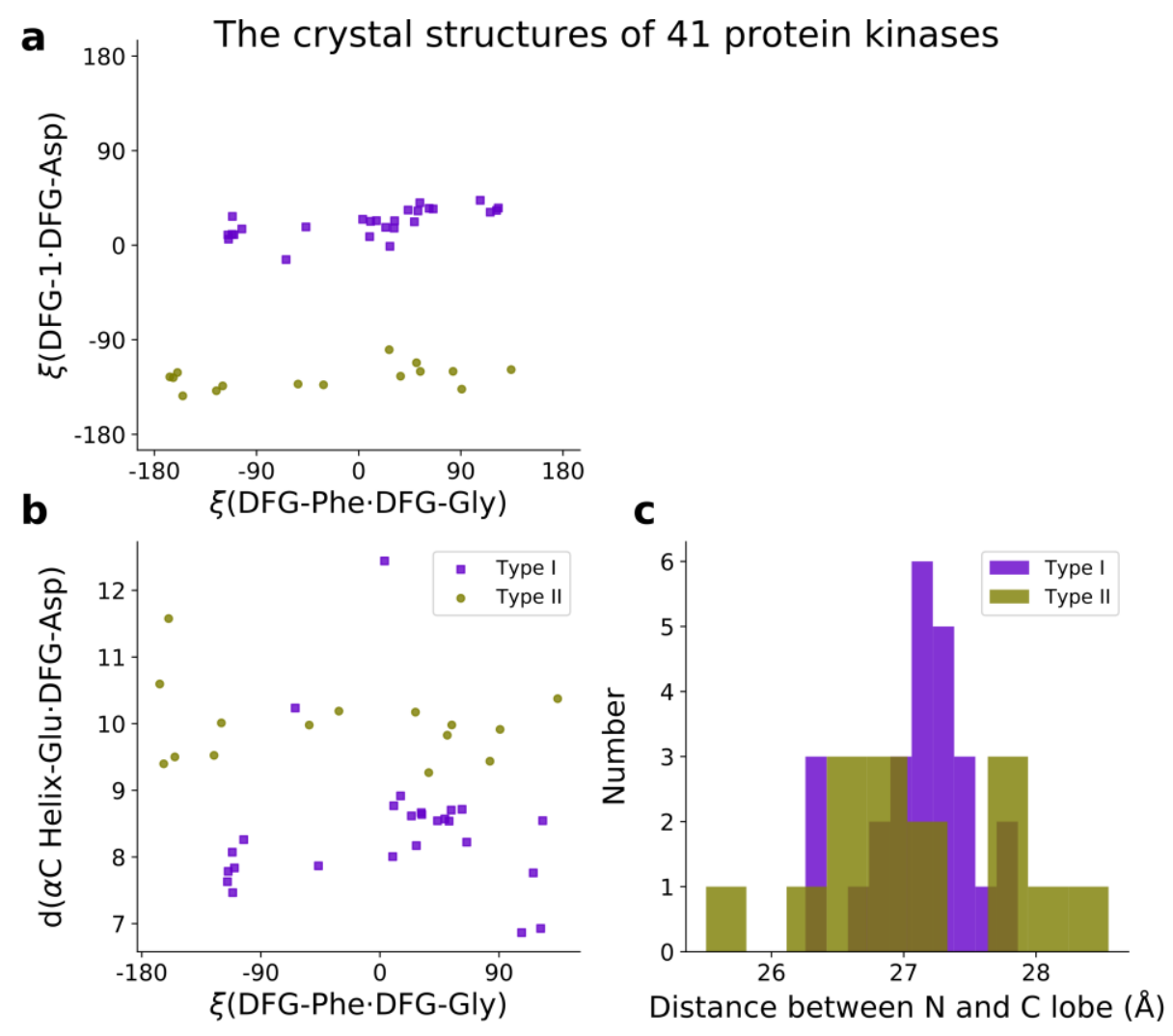

Figure S7. The structural characteristics of 41 protein kinase in crystal structures.

(a) The $\xi$ is the pseudodihedral angle of four consecutive alpha-carbon atoms.

DFG-Phe.DFG-Gly is the short form of DFG-Asp, DFG-Phe, DFG-Gly, and DFG +1 residues; DFG-1-DFG-Asp is DFG-2, DFG-1, DFG-Asp, and DFG-Phe residues. (b) $d(\alpha C$ Helix-Glu-DFG-Asp) is the distance between the alpha-carbon atoms of $\alpha$ C Helix-Glu and DFG-Asp residues. (c) The distribution of the distance between $\mathrm{N}$ - and $\mathrm{C}$-lobe of Type I and Type II inhibitors-bound kinases. 


\section{Part 7. A small-scale comparison of local-scaled MD and TRAMD}

In order to make a comparison of the outcomes of local-scaled MD and other methods, we have chosen $\tau$ RAMD in GROMACS v2020.2, which is available to us and relatively convenient to apply, to simulate the dissociation of four kinase complexes, including 4V01 (Type II inhibitor, koff = 1.9e-4 s-1), 5TBE (Type I inhibitor, koff = 7.9e-4 s-1), 4PMM (Type II inhibitor, koff = 1.7e-3 s-1), and 5E1S $($ Type I inhibitor, koff $=1.7 \mathrm{e}-2$ s-1). The parameter settings adopted by Kokh et al. in their work (J. Chem. Theory Comput. 2018, 14, 3859-3869, ramd-force = 14 kcal mol-1 Ang-1) were also used in this trial. In each case, twenty $\tau$ RAMD simulations were conducted from different starting configurations. Those starting configurations were obtained from NVT simulation as described in our manuscript. The full length of each $\tau$ RAMD trajectory was set to $50 \mathrm{~ns}$. If the minimal distance between the protein and the ligand exceeded 5 $\AA$, the $\tau$ RAMD simulation was terminated prematurely. We calculated the relative residence time by using the "tauRAMD-v2.py" script provided by Wade's group. The results are summarized together with the binding energy integrals given by our localscaled MD simulation in Table S3.

Based on the results, if one chooses a proper protonation state in each complex, both $\tau$ RAMD and local-scaled MD can produce the correct rank of the koff values of the four select complexes. Nevertheless, the averaged binding energy integral by our localscaled MD method produces a better linear relationship than the averaged relative residence time by $\tau$ RAMD (see Figure S8). 
Table S3. Dissociation rate constants of four selected complexes along with the relative residence time given by TRAMD and the integrals of binding energy given by our local-scaled MD

\begin{tabular}{|c|c|c|c|c|}
\hline PDB entry & $\begin{array}{c}\text { Inhibitor } \\
\text { type }\end{array}$ & $\begin{array}{c}\text { Relative residence } \\
\text { time given by } \\
\text { TRAMD }\end{array}$ & $\begin{array}{c}\text { Integral of } \\
\text { negative binding } \\
\text { energy } \\
\left(10^{3} \mathrm{kcal} / \mathrm{mol}\right) \text { by } \\
\text { local-scaled MD }\end{array}$ & koff (s-1) \\
\hline 4V01_P1 & $\|$ & $2.9 \pm 0.71$ & $-134.99 \pm 12.22$ & $1.9 \mathrm{e}-4$ \\
\hline 4V01_P2 & $\|$ & $2.03 \pm 0.22$ & $-130.94 \pm 13.86$ & $1.9 \mathrm{e}-4$ \\
\hline 5TBE_P1 & $\mathrm{I}$ & $2.3 \pm 0.22$ & $-114.81 \pm 14.02$ & $7.9 \mathrm{e}-4$ \\
\hline 5TBE_P2 & $\mathrm{I}$ & $4.01 \pm 0.38$ & $-110.47 \pm 13.53$ & $7.9 \mathrm{e}-4$ \\
\hline 4PMM_P1 & $\|$ & $0.32 \pm 0.02$ & $-110.72 \pm 10.12$ & $1.7 \mathrm{e}-3$ \\
\hline 4PMM_P2 & $\|$ & $0.12 \pm 0.01$ & $-78.57 \pm 11.97$ & $1.7 \mathrm{e}-3$ \\
\hline 5E1S_P1 & $\mathrm{I}$ & $0.09 \pm 0.00$ & $-69.98 \pm 7.69$ & $1.7 \mathrm{e}-2$ \\
\hline
\end{tabular}

local-scaled MD (5 ns)

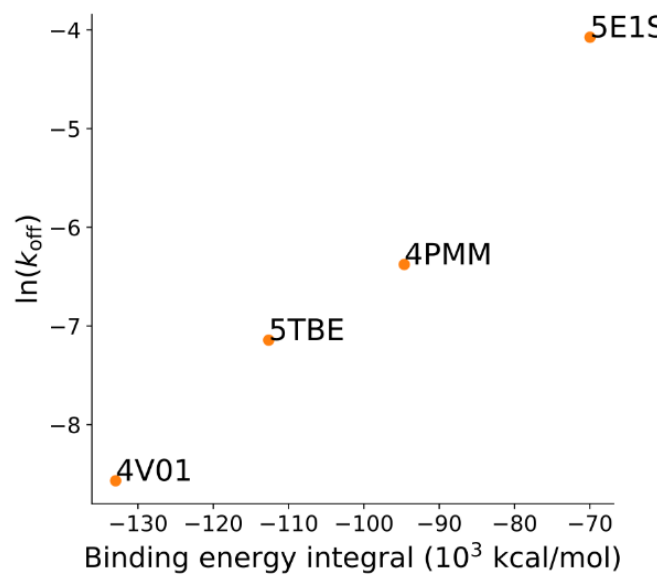

(a)
tRAMD (force $=14 \mathrm{kcal} \mathrm{mol}-1$ Ang-1)

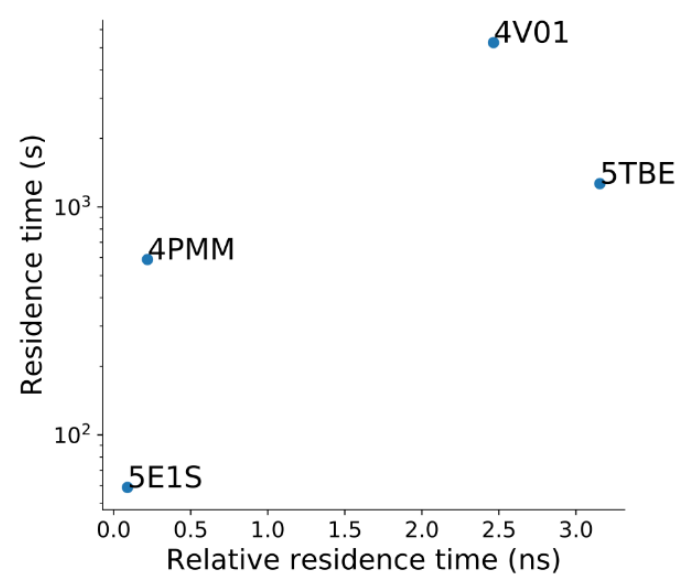

(b)

Figure S8. Experimental $\ln \left(k_{\text {off }}\right)$ values versus (a) binding energy integral given by local-scaled MD simulation, and (b) relative residence time by TRAMD simulation. 
Besides examining the correlation between computed results and the experimental $\ln \left(k_{\text {off }}\right)$ values, we also compared the simulation outcomes of $\tau$ RAMD and local-scaled MD for the four selected complexes. First, let us examine the RMSD values of the ligand during simulation (Figure S9). One can see that although $\tau$ RAMD tends to differentiate fast leaving inhibitors (e.g. 5E1S, koff =1.7e-2) and slow leaving inhibitors (e.g. $4 \mathrm{~V} 01,1.9 \mathrm{e}-4)$, the dissociation event typically occurs in a sudden manner, which is indicated by the long vertical lines in Figure S9. In fact, the complex structure under $\tau$ RAMD simulation remains relatively stable during simulation. In this sense, one would suspect if $\tau$ RAMD is able to sufficiently sample the possible intermediate states on the ligand dissociation pathway. In contrast, the change in ligand RMSD is generally much gentler by our local-scaled MD simulation. Thus, local-scaled MD is hopefully more capable for sampling the intermediate states in dissociation.

Second, let us examine the local and global conformational changes in protein structure (Figure S10). Here, one can observe more clearly that the protein structure is relatively stable during $\tau$ RAMD simulation. In the case of local-scaled MD simulation, generally a larger region in the protein conformational space is explored. It is not difficult to understand the difference between the basic concept of $\tau$ RAMD and local-scaled MD. The basic concept of $\tau$ RAMD is like a particle undergoing the Brownian movement, where a random force is constantly applied to the particle (i.e. the ligand under simulation). Due to the random nature of the applied force, its influence on the particle movement is largely canceled out, and thus the net shift of the particle is not obvious unless a significant amount of simulation time is allowed. In a local-scaled MD simulation, the attractive force between protein and ligand is gradually reduced while the repulsive force remains. As result, when the ligand gets close to the protein, before and after it escapes from the binding pocket, the protein structure has to respond to push the ligand away. Also, this breath-like conformational motion allows water molecules to interfere the interactions between the protein and the ligand, which speeds up the ligand dissociation process. 
It should be emphasized that molecular modeling of ligand binding/unbinding is still a pre-mature field. Technically, it is difficult to compare those enhanced MD sampling methods, such as metadynamics, scaledMD, and $\tau$ RAMD, in a rigorous manner. Each method follows a certain type of assumption, and there is no widely accepted benchmark designed for such a comparison. Therefore, although a good number of studies of this type have been published in literature, to the best of our knowledge, no one has conducted a serious comparison of different methods even on a limited number of samples. Here, we have attempted to compare $\tau$ RAMD and local-scaled MD on four selected complexes. A more meaningful comparison of course needs another level of effort. 
4V01 P1 (Type II inhibitor)

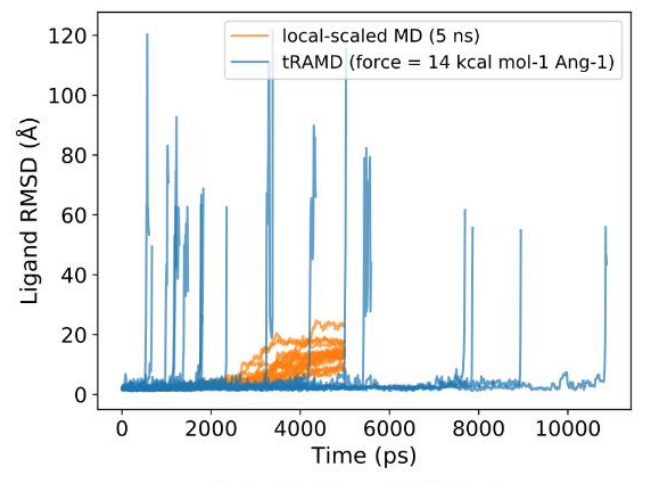

5TBE_P1 (Type I inhibitor)

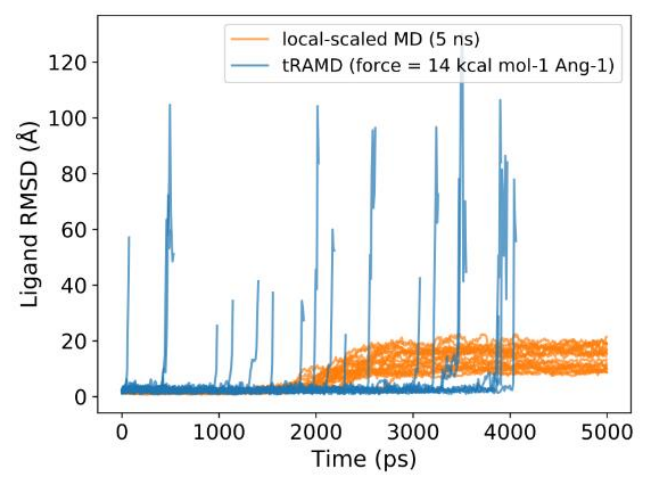

4PMM_P1 (Type II inhibitor)

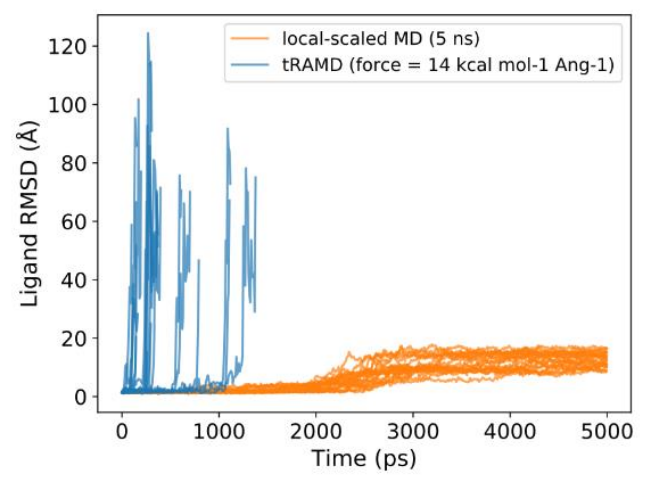

5E1S_P1 (Type I inhibitor)

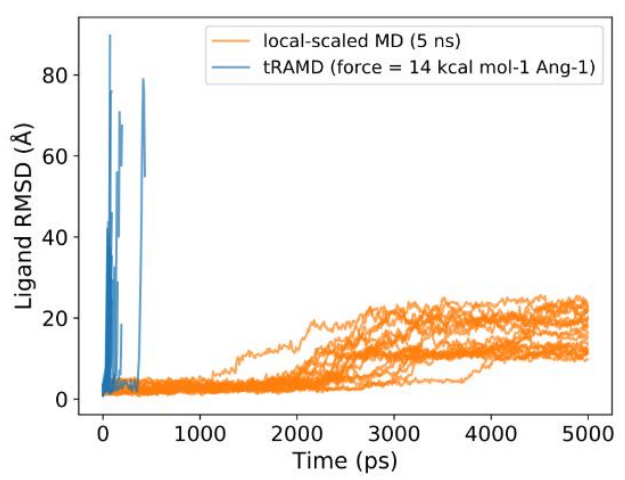

4V01 P2 (Type II inhibitor)

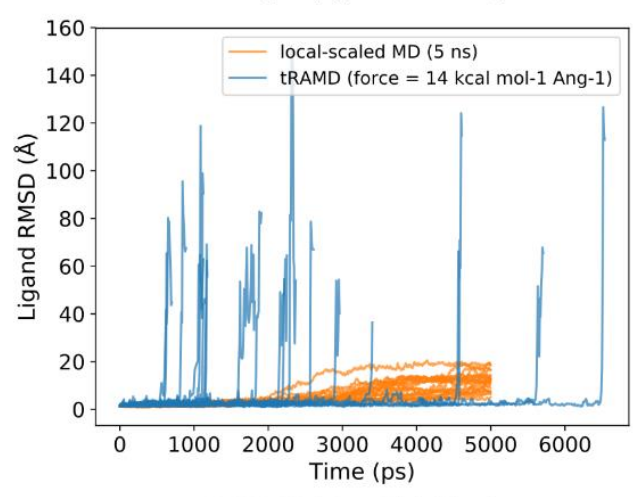

5TBE P2 (Type I inhibitor)

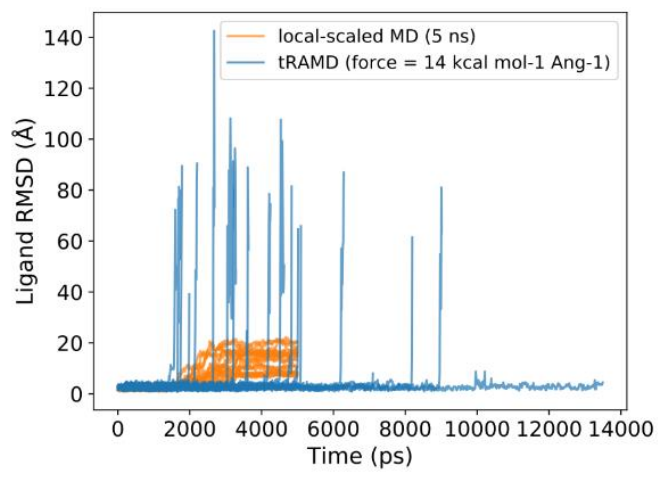

4PMM P2 (Type II inhibitor)

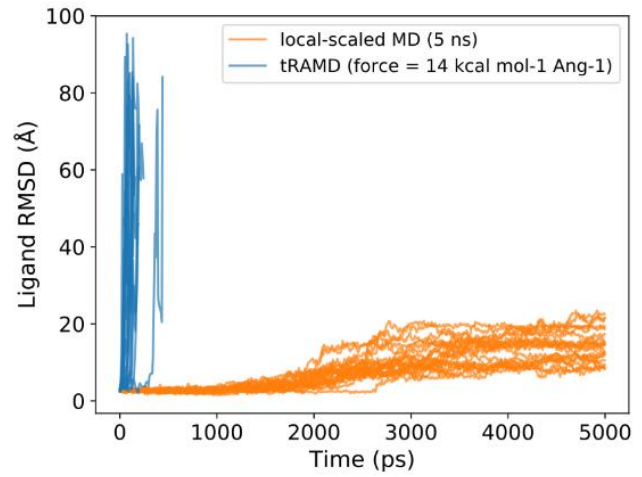

Figure S9. Ligand RMSD values observed during the simulation of local-scaled MD (orange) and TRAMD (blue) for four selected kinase-inhibitor complexes. 

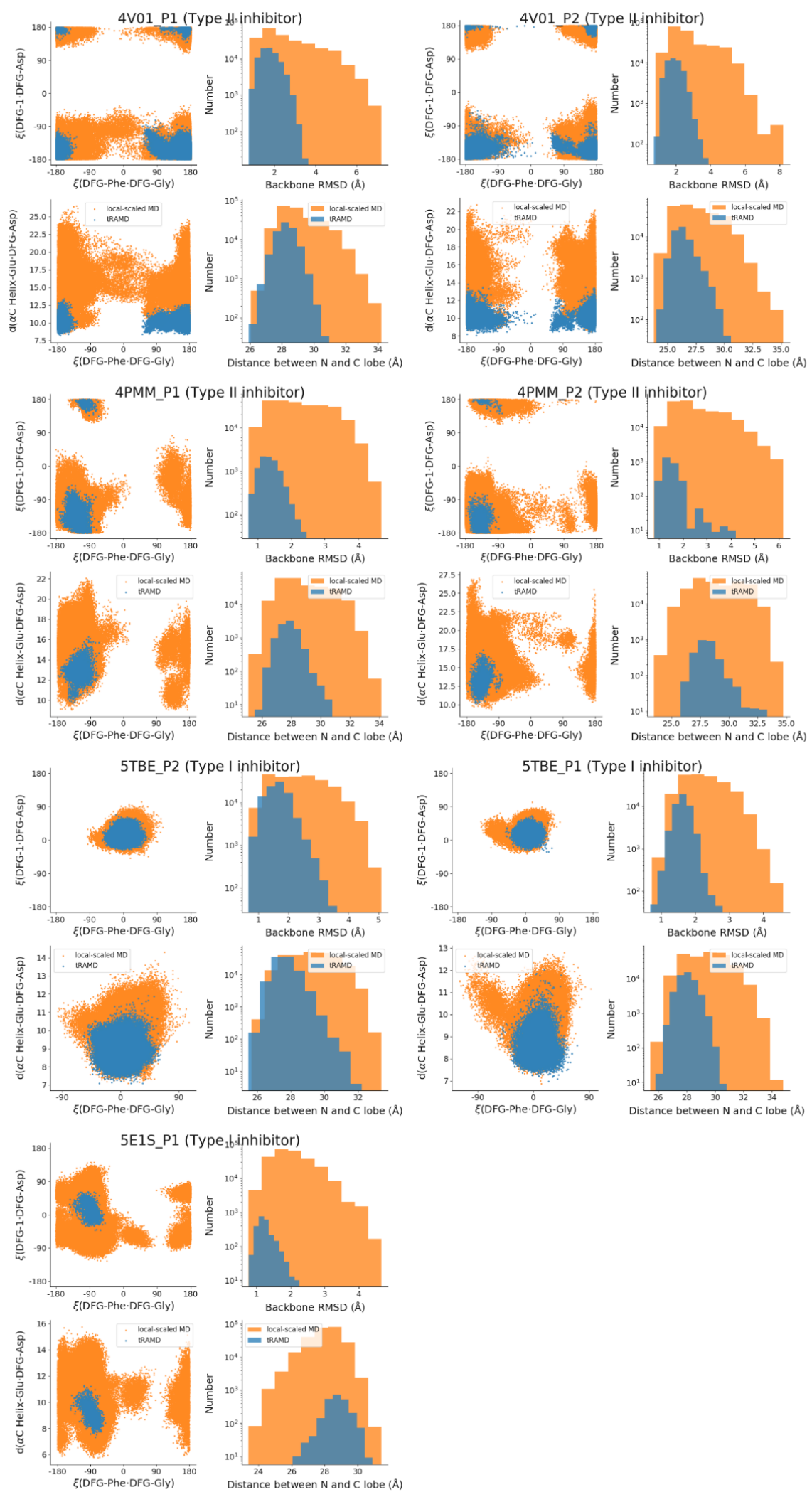

Figure S10. Protein conformational landscapes of four kinase-inhibitor complexes depicted by local-scaled MD (orange) and tRAMD (blue). The results of each complex come from 20 replicas of simulation. 


\section{Part 8. Residence time of water molecules}

Figure S11. The residence time of water molecules within $5 \AA$ of the ligand in local-scaled MD. The median value of water residence time is showed in pink. Since no water in complex 3HEG_P1 and 5MTY_P1 endured the preliminary MD process, they have no detailed water residence information.
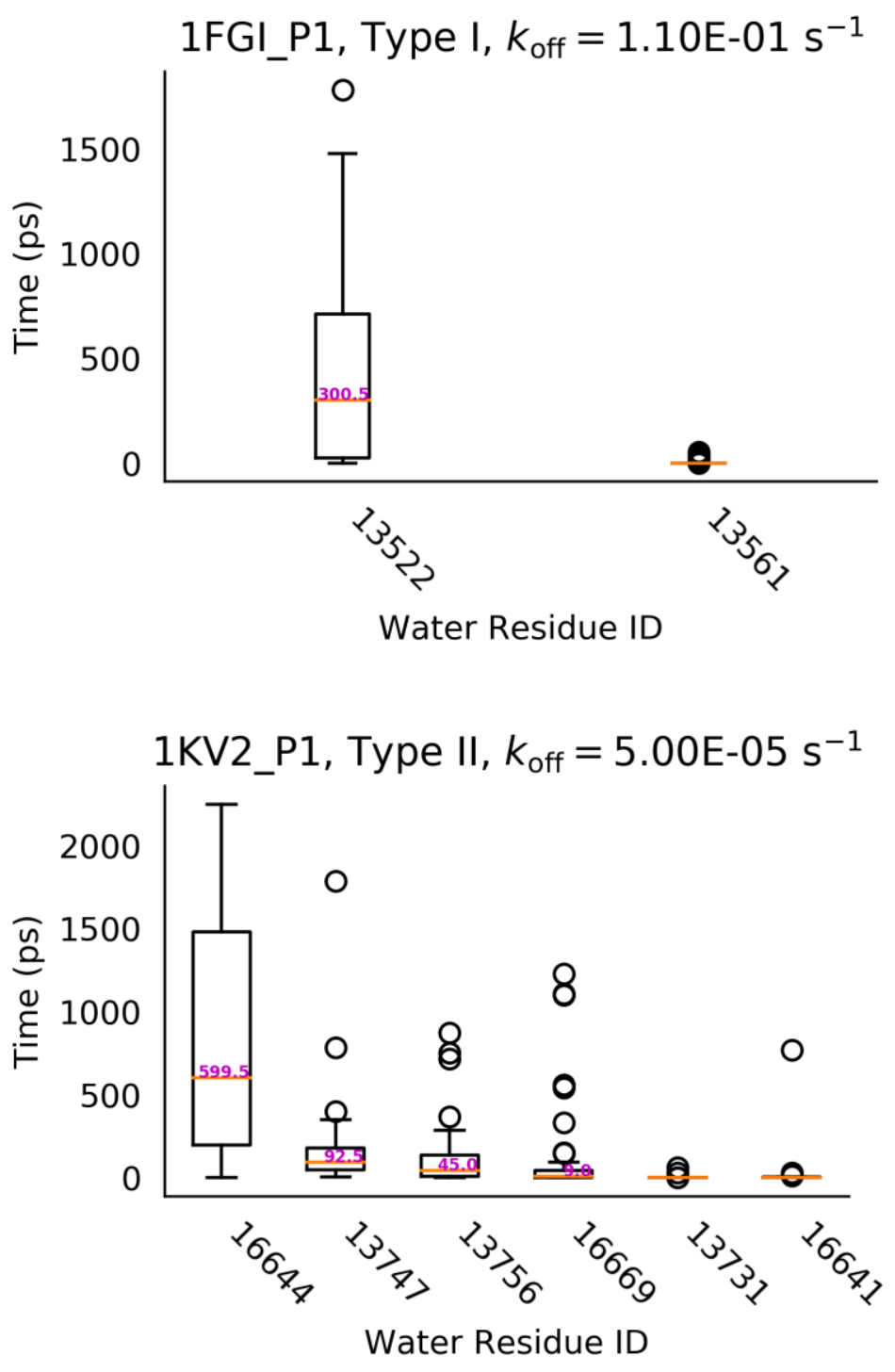

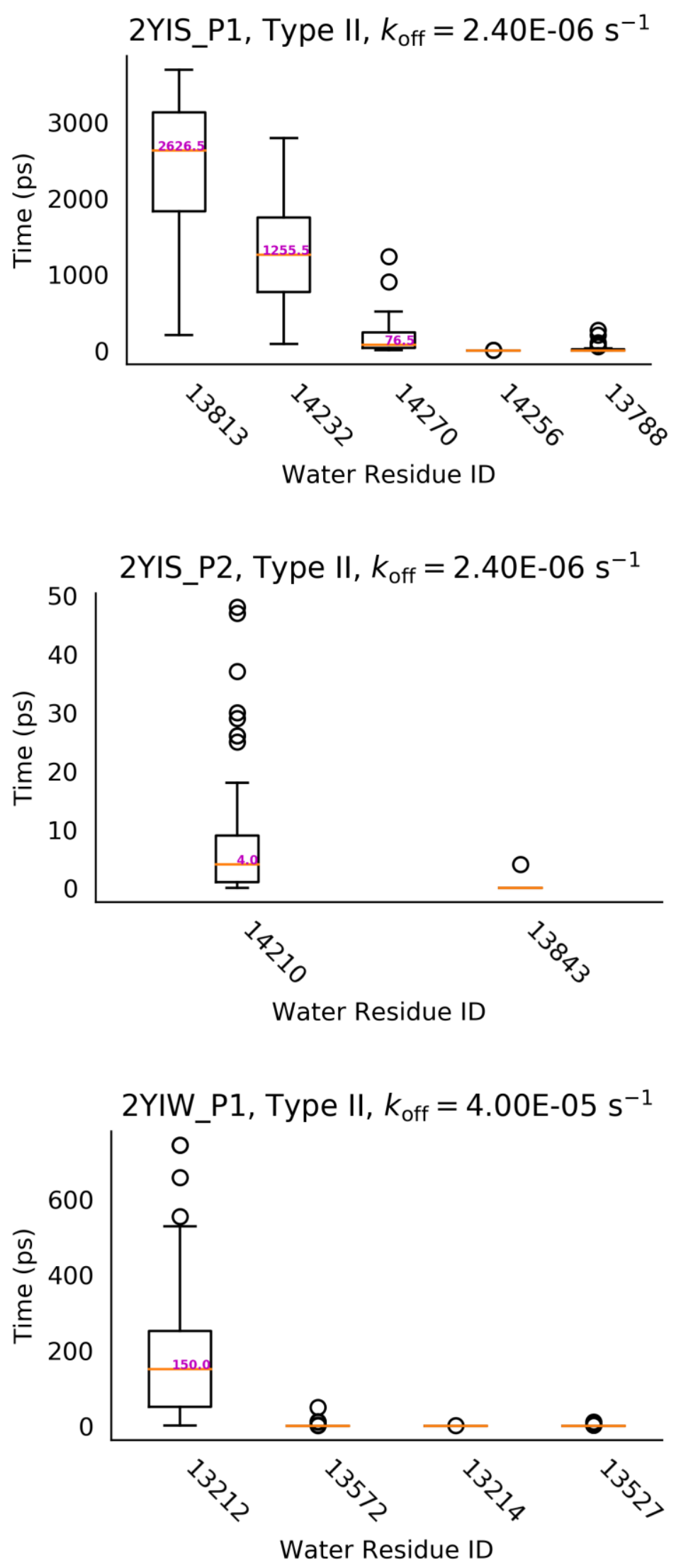

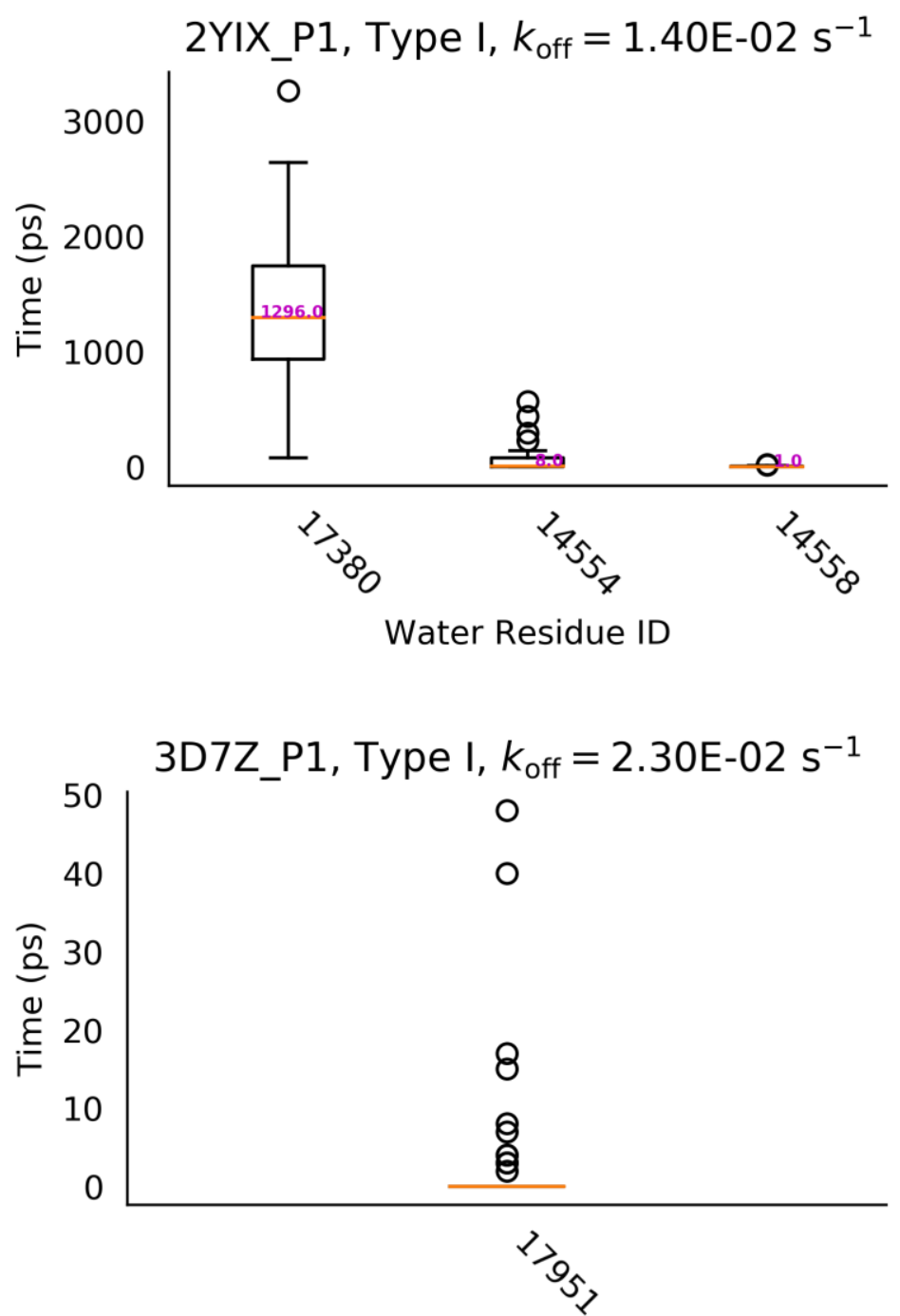

Water Residue ID

3D83_P1, Type II, $k_{\text {off }}=8.40 \mathrm{E}-03 \mathrm{~s}^{-1}$

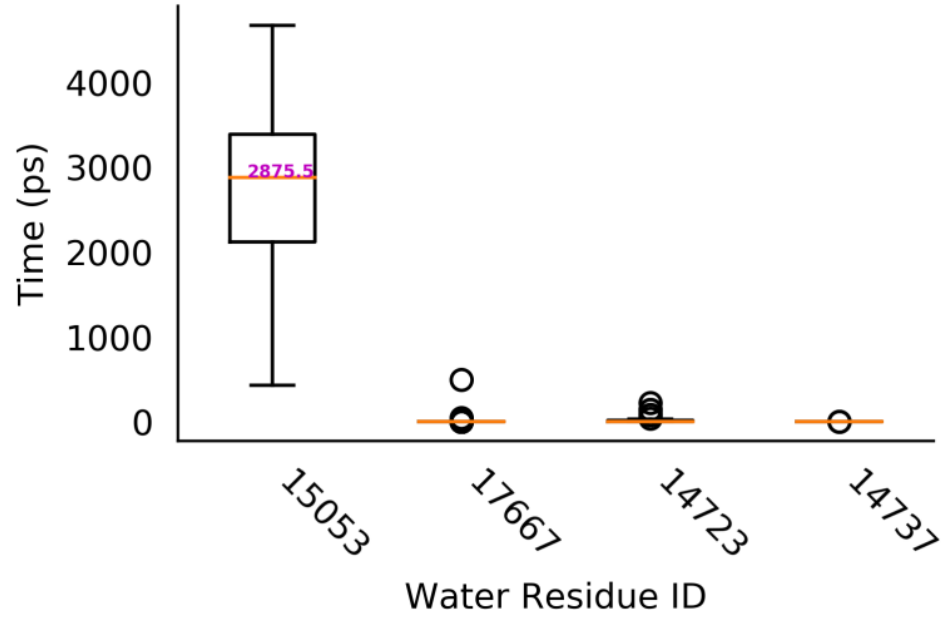



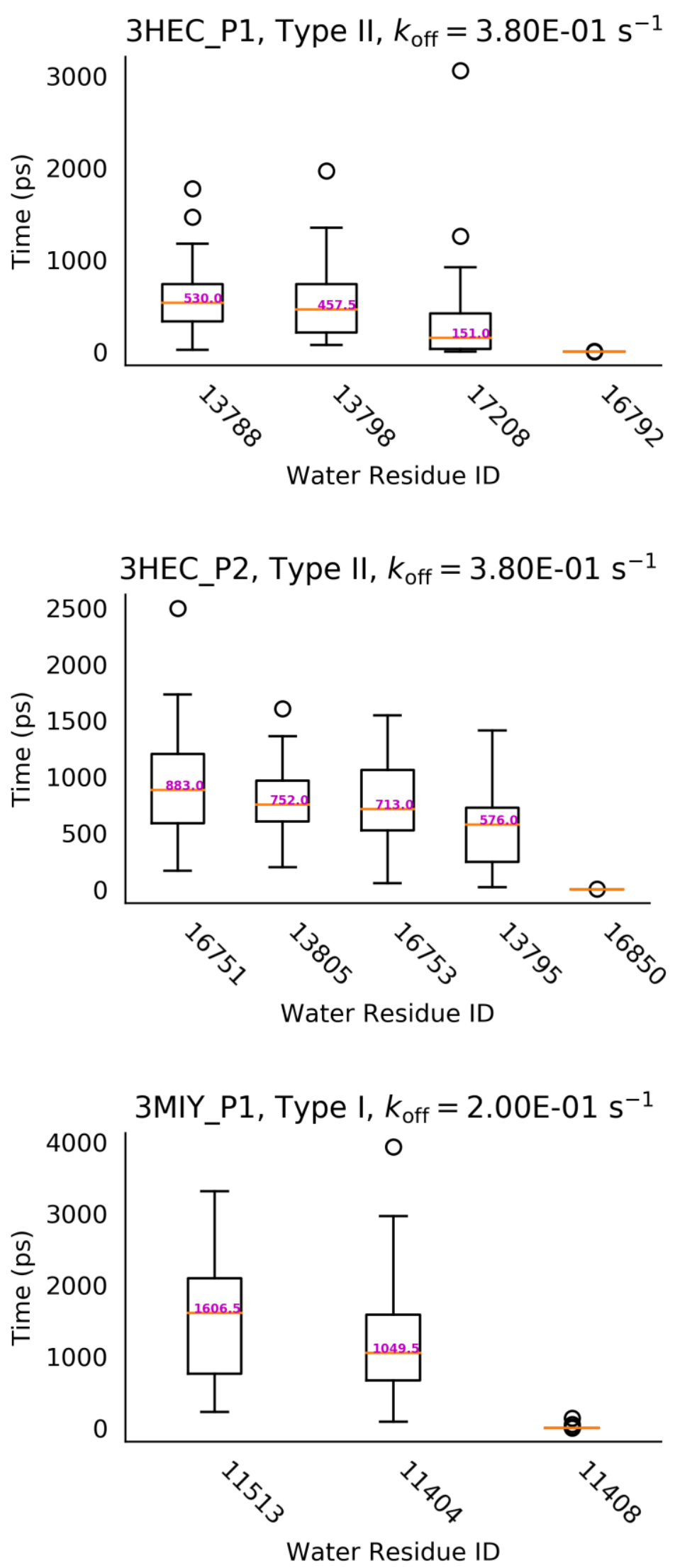


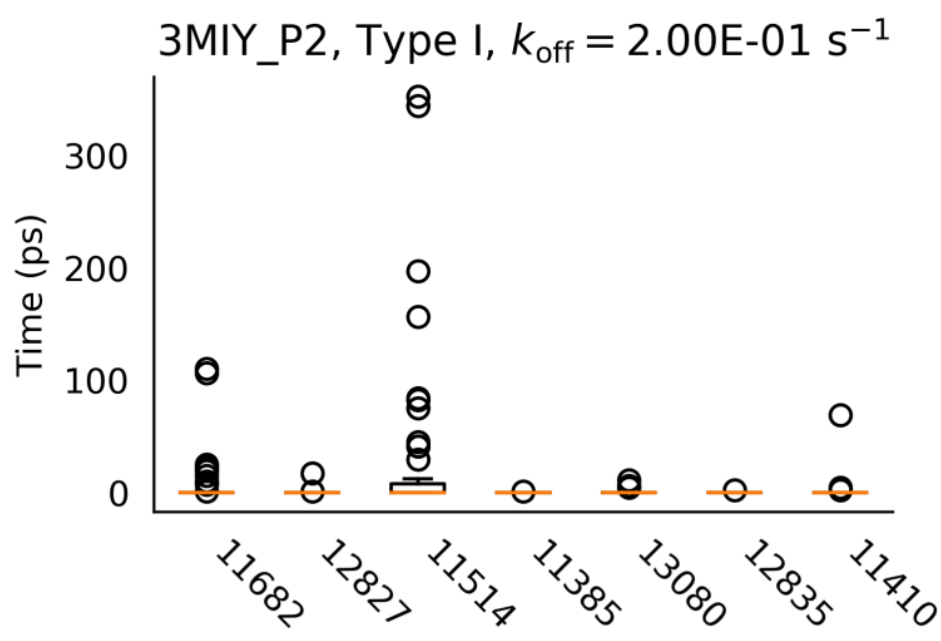

Water Residue ID

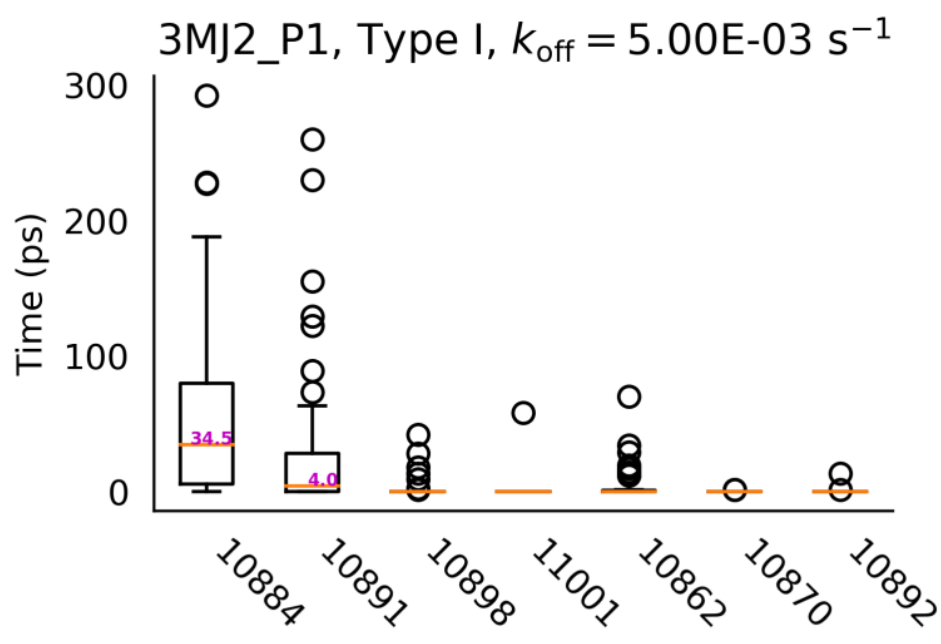

Water Residue ID

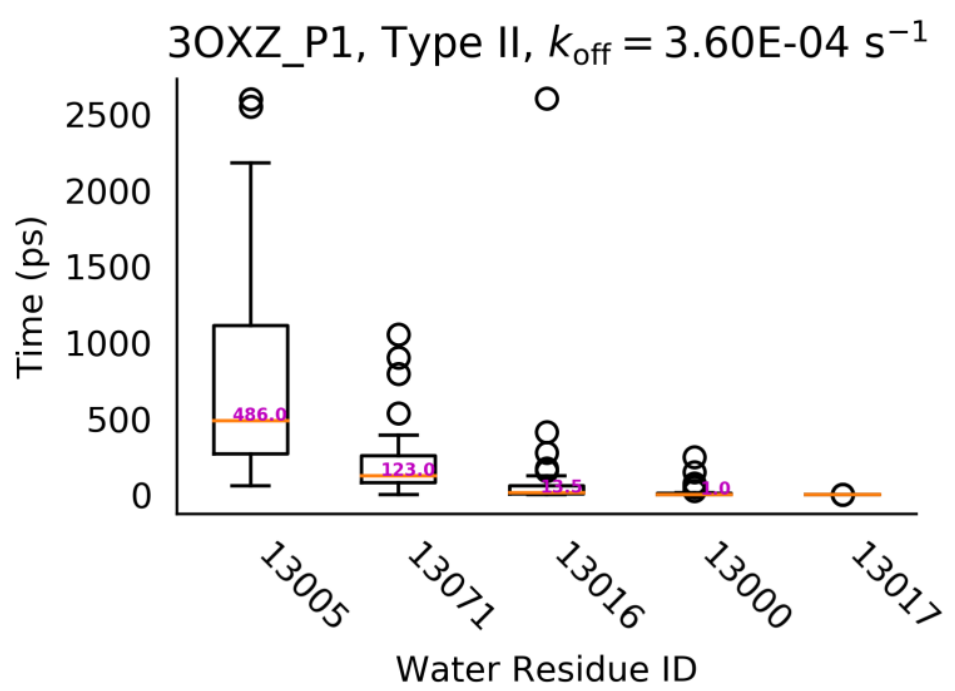

68 

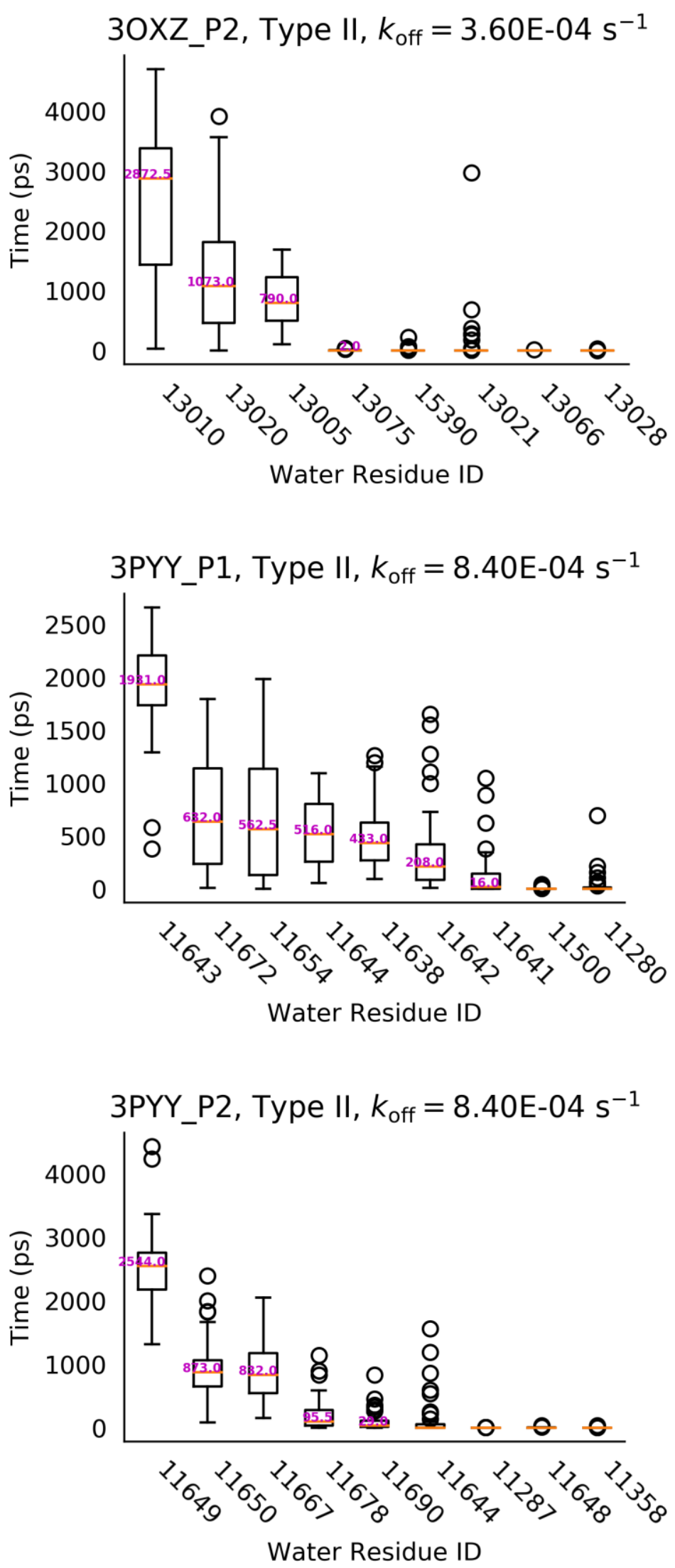

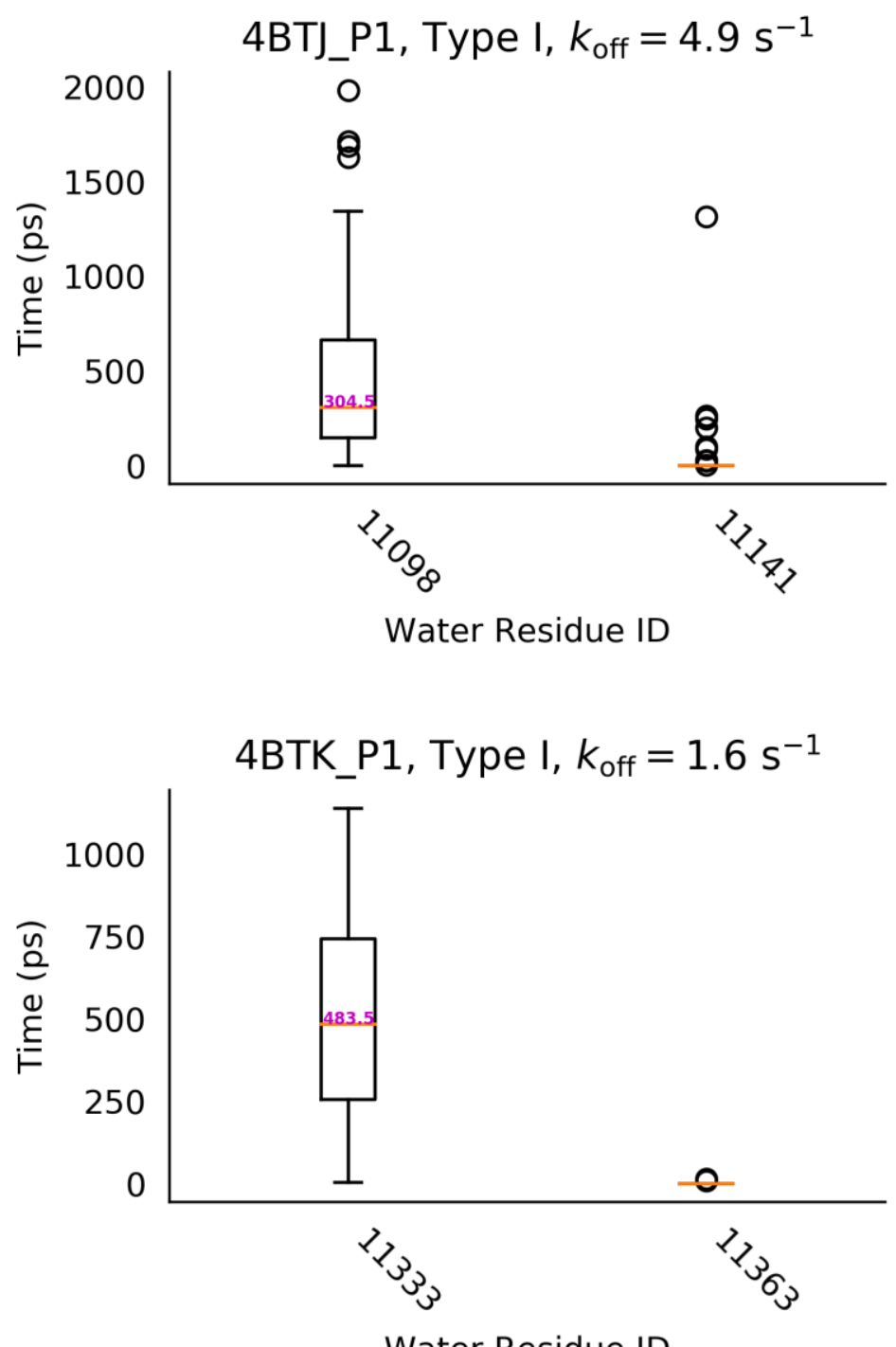

Water Residue ID

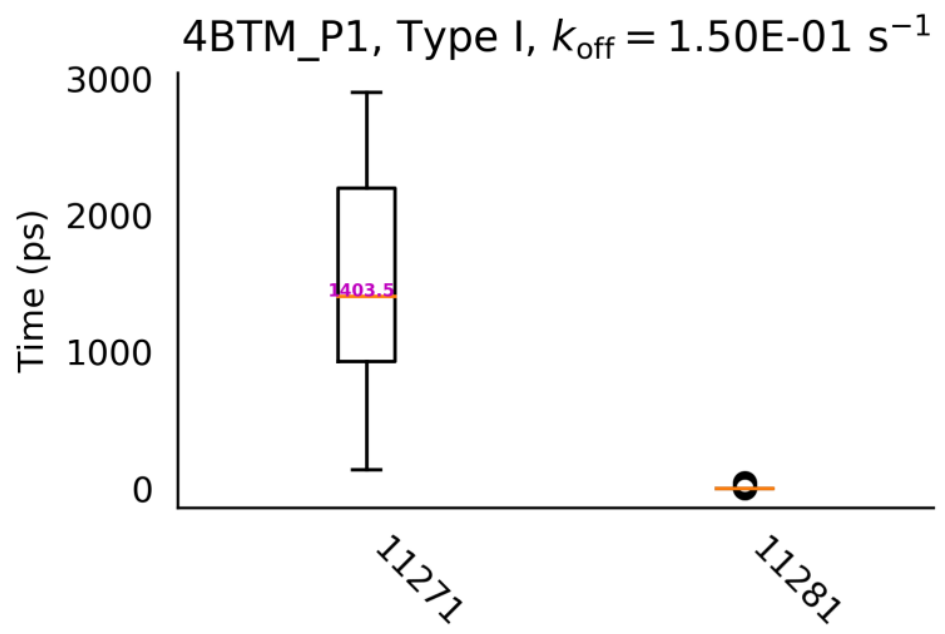

Water Residue ID 


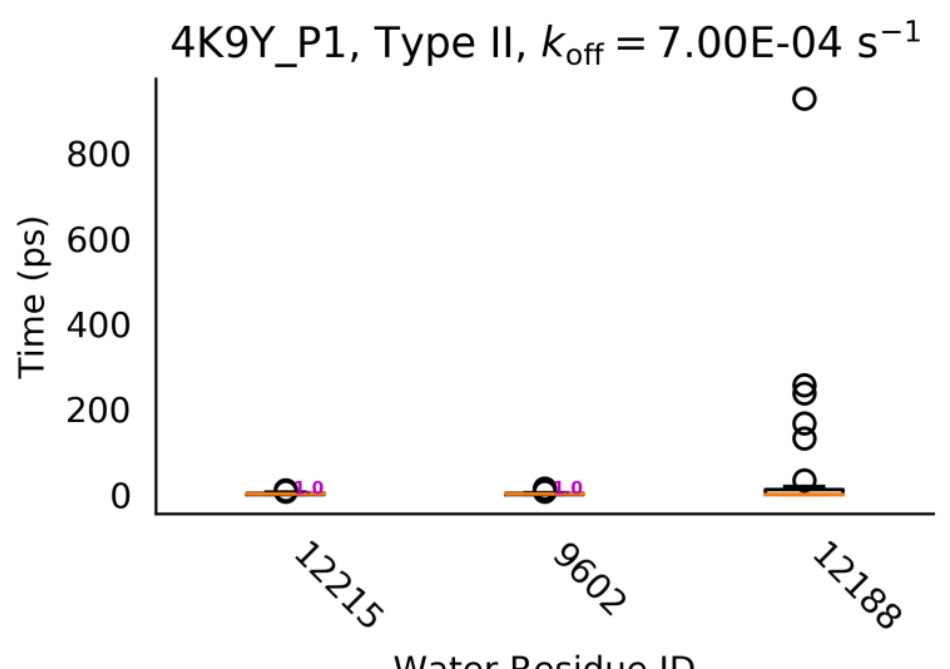

Water Residue ID
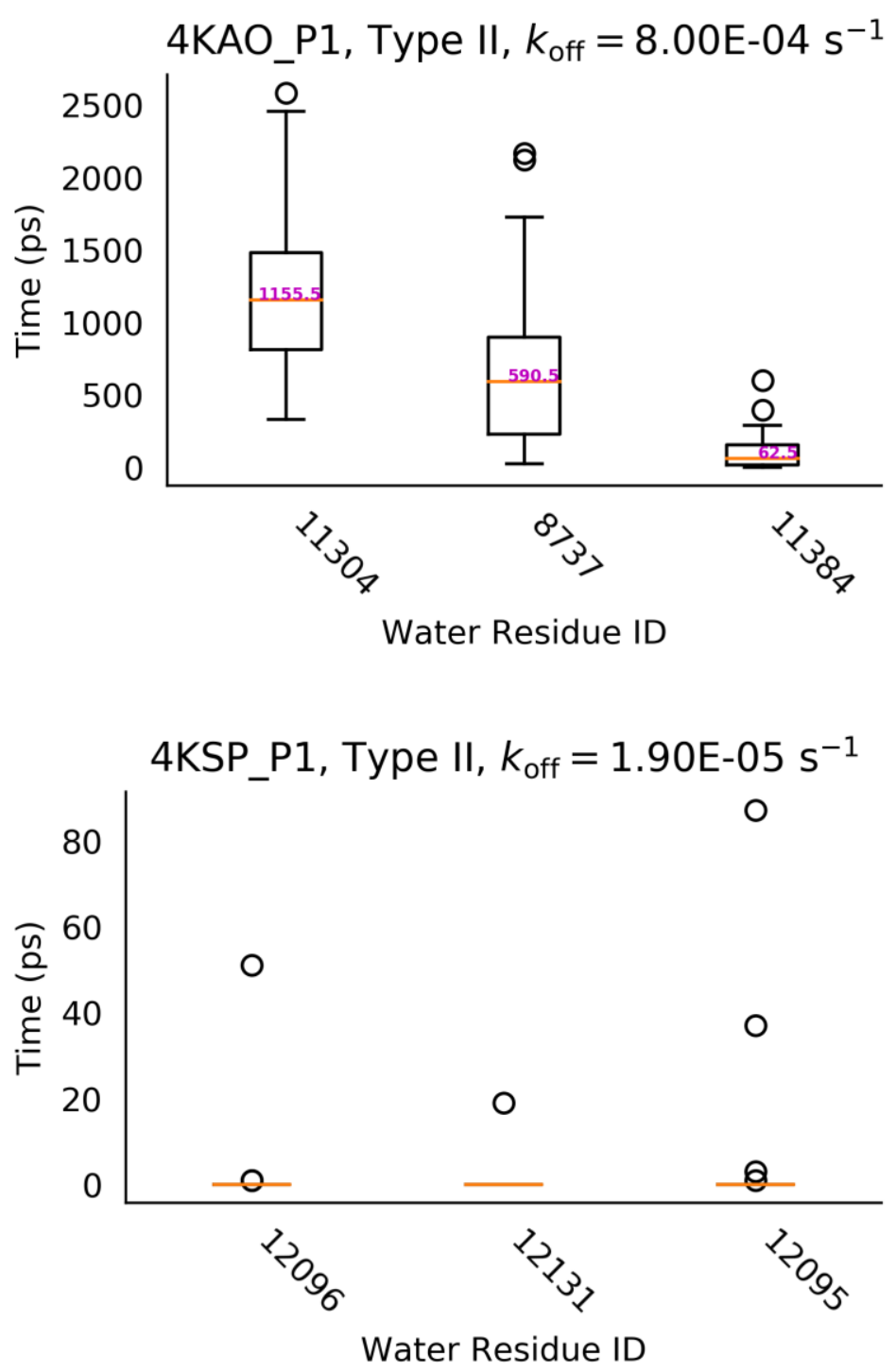

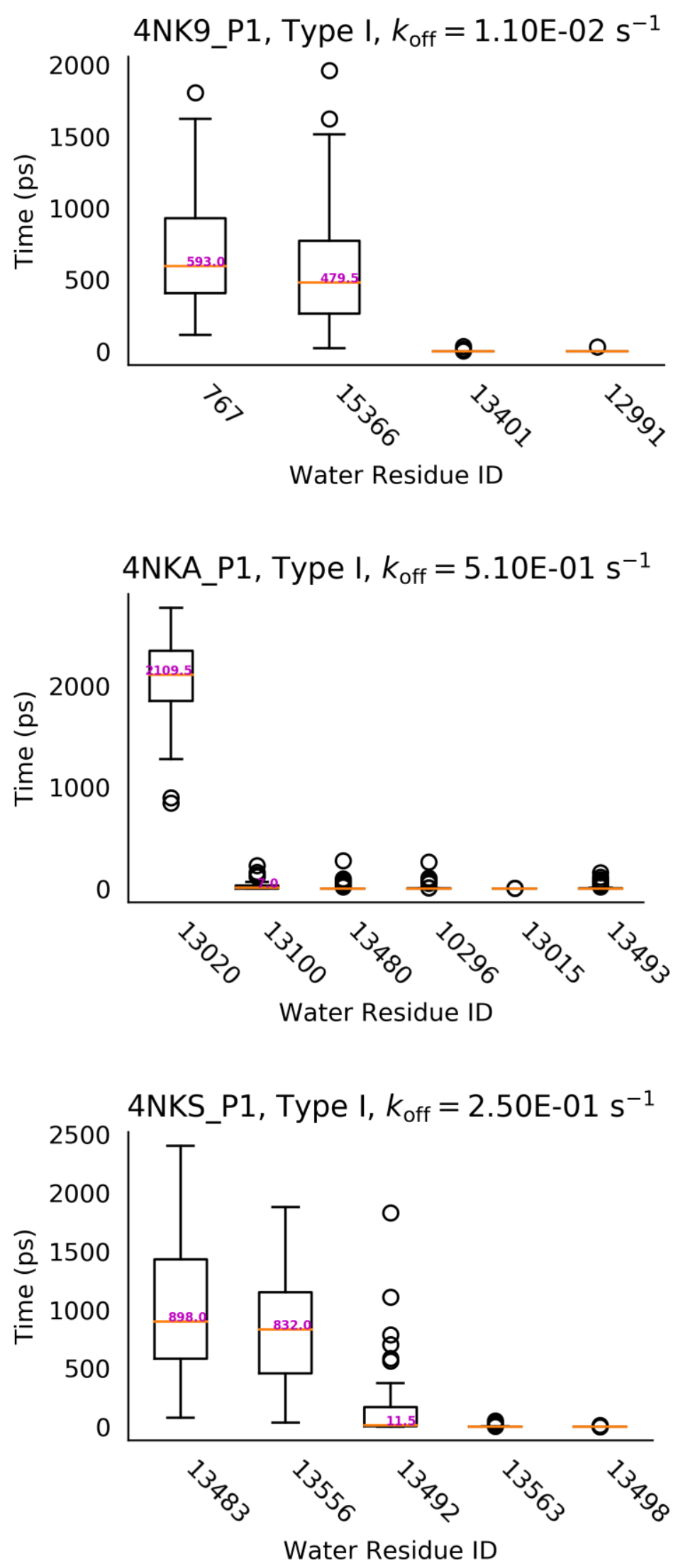

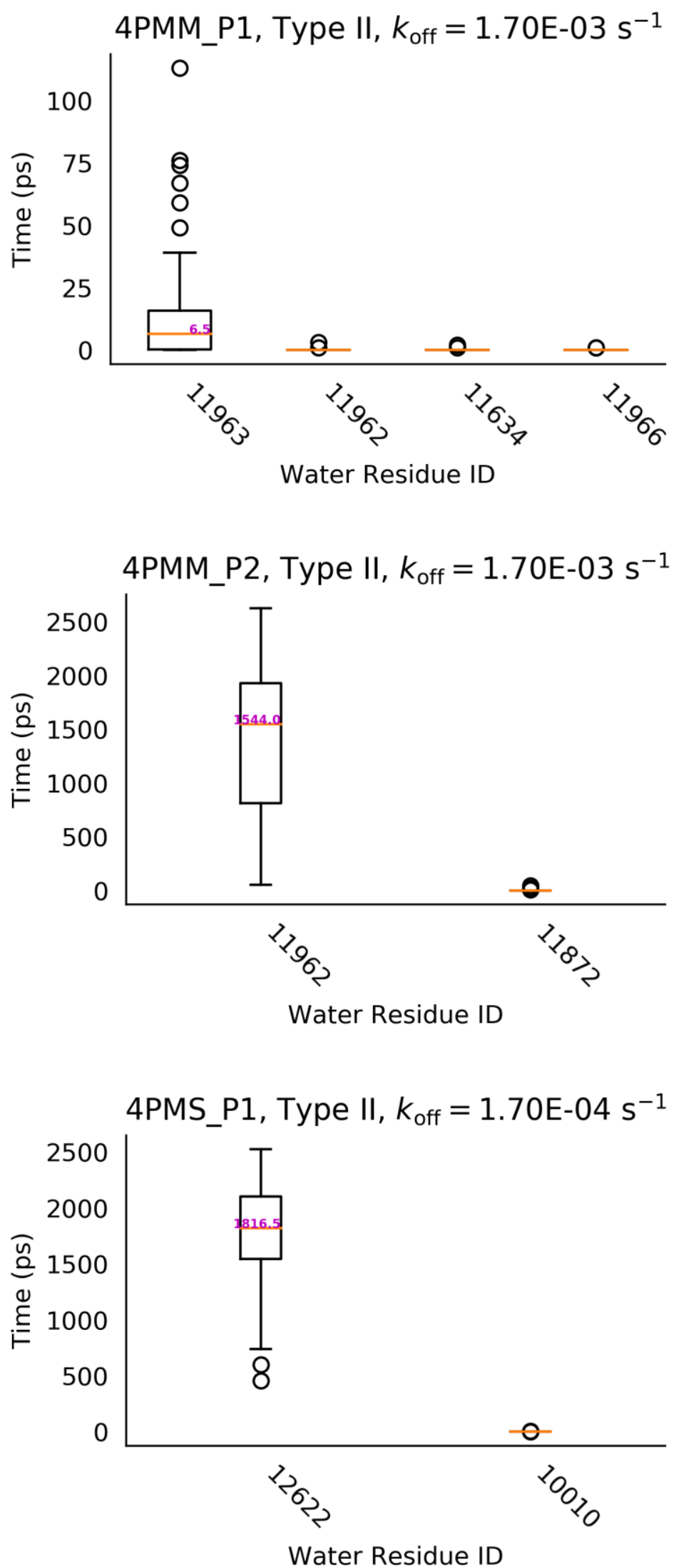

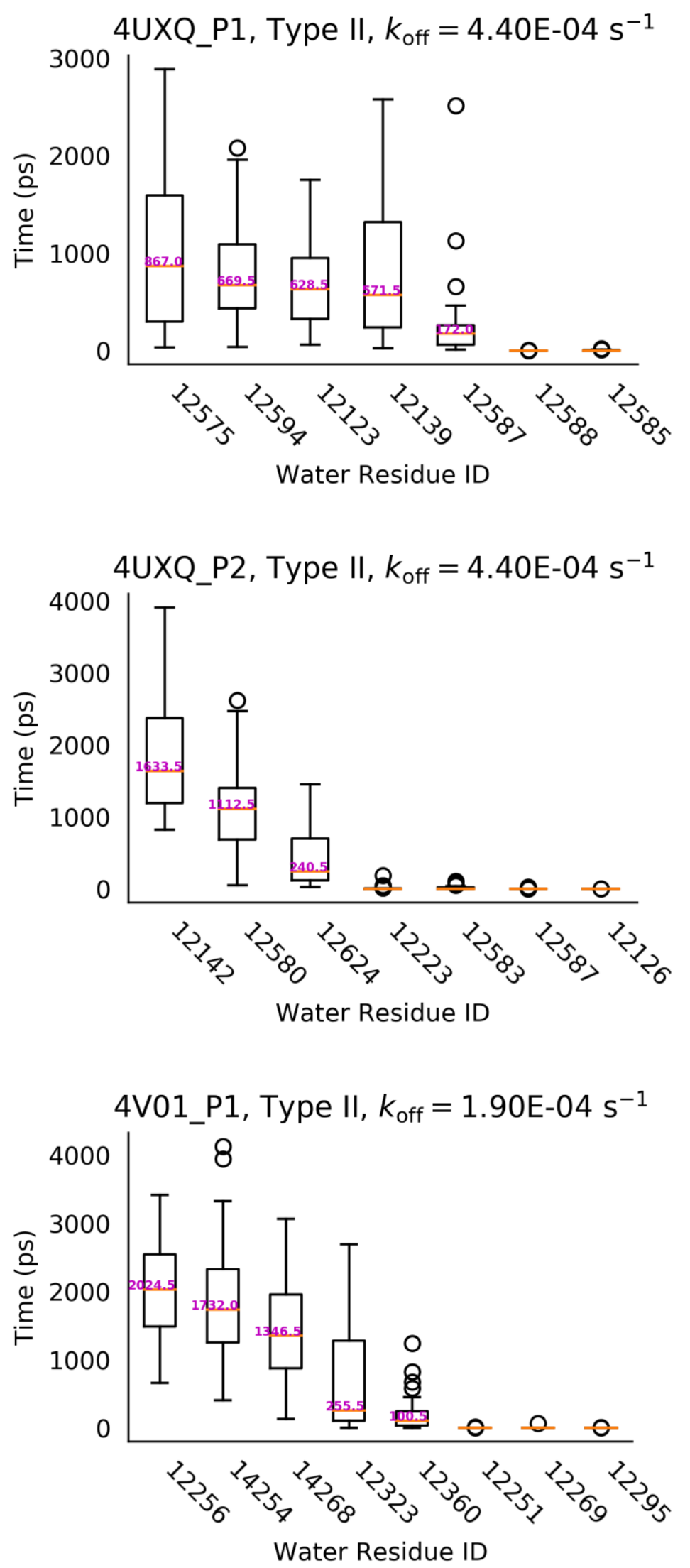

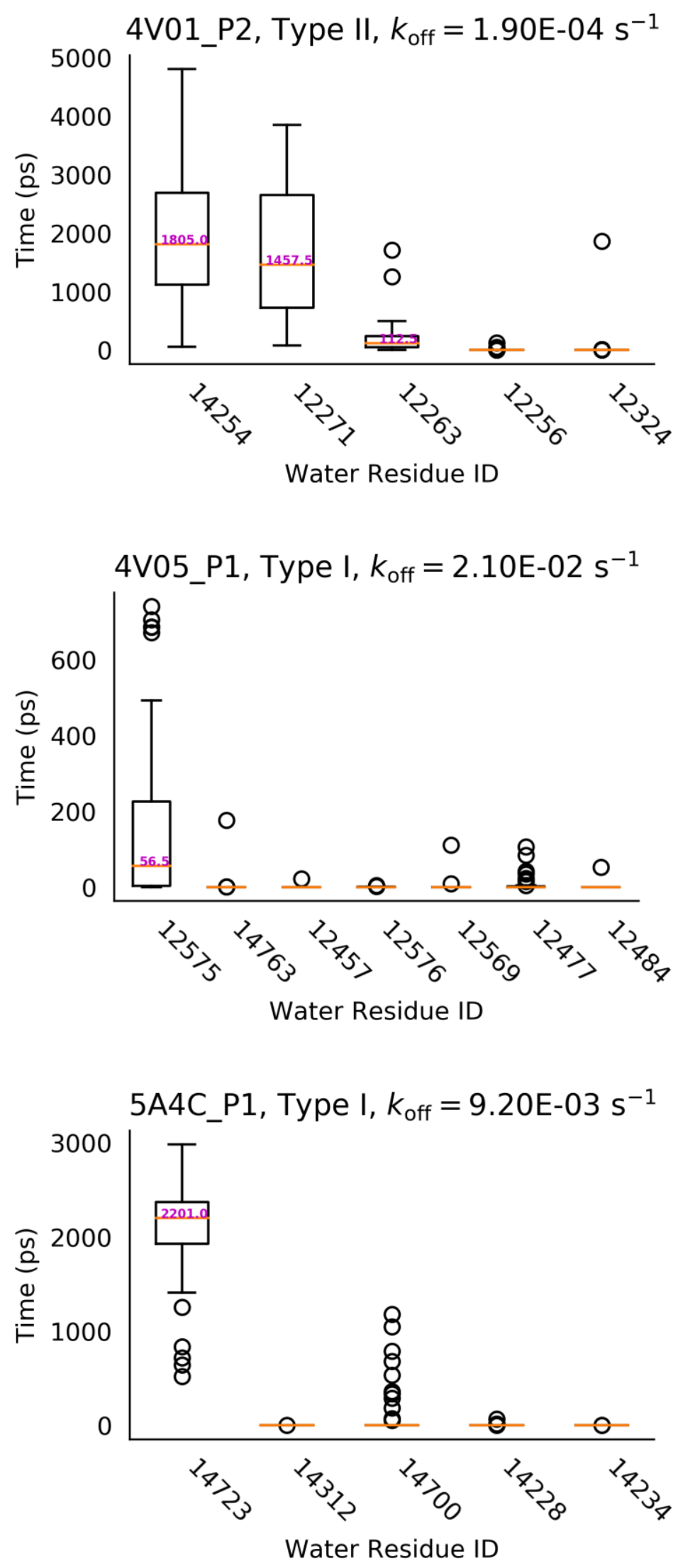

75 

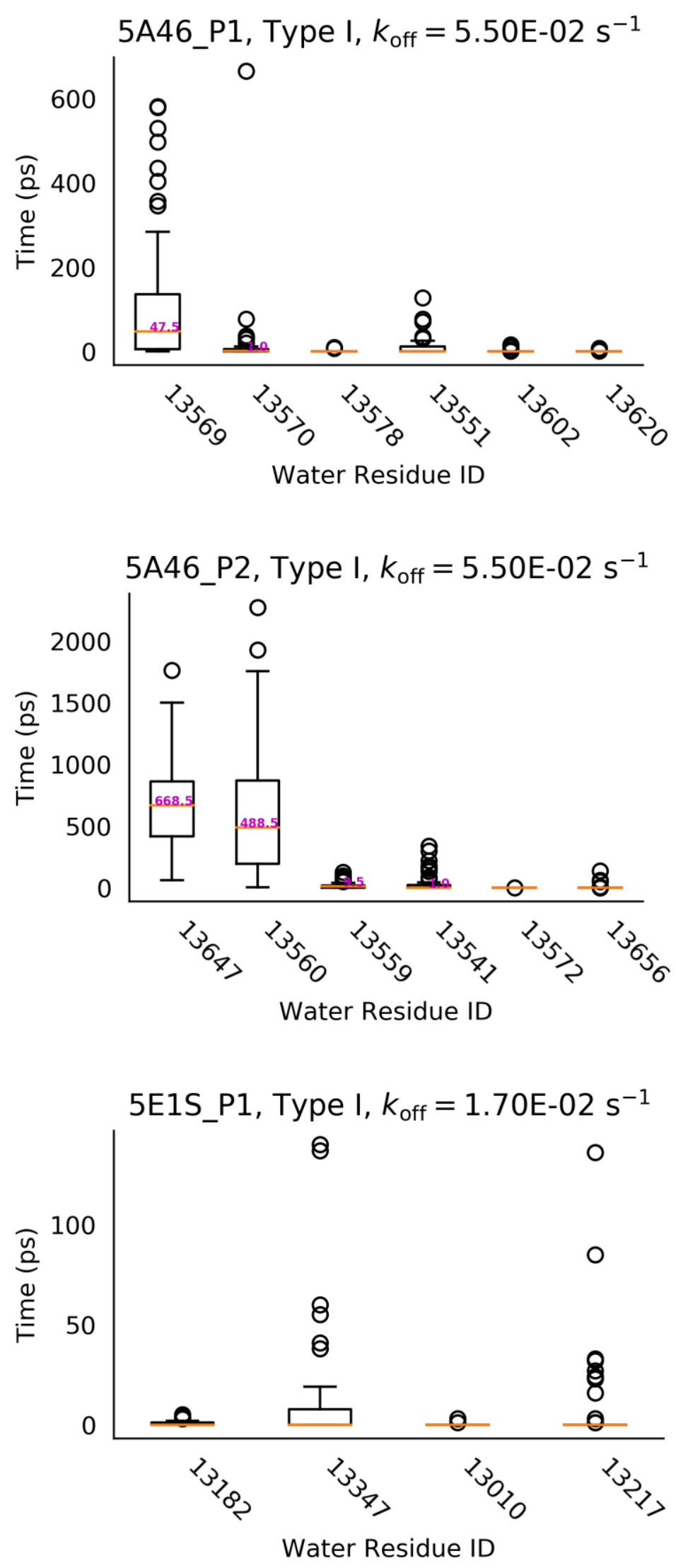

76 


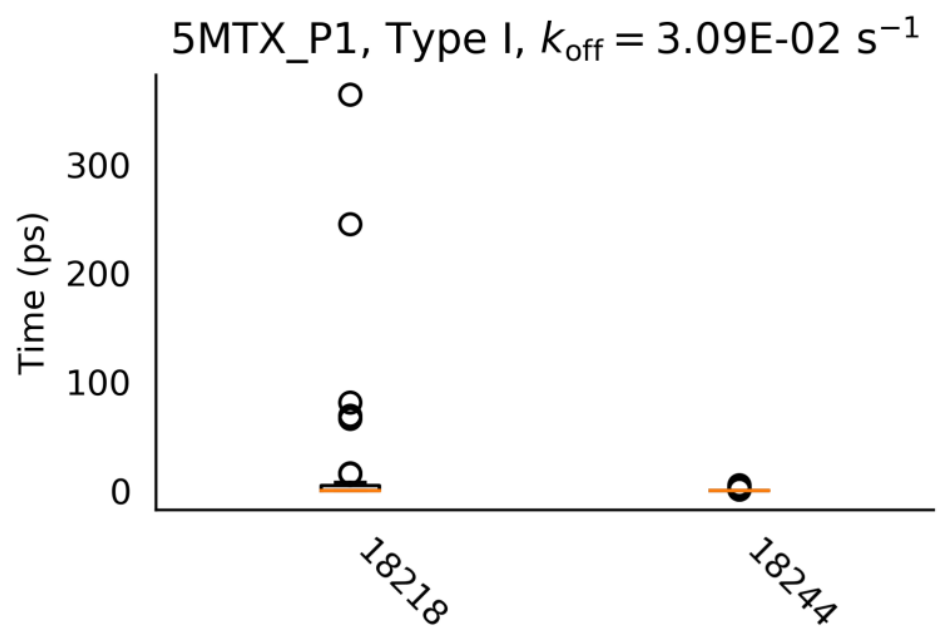

Water Residue ID

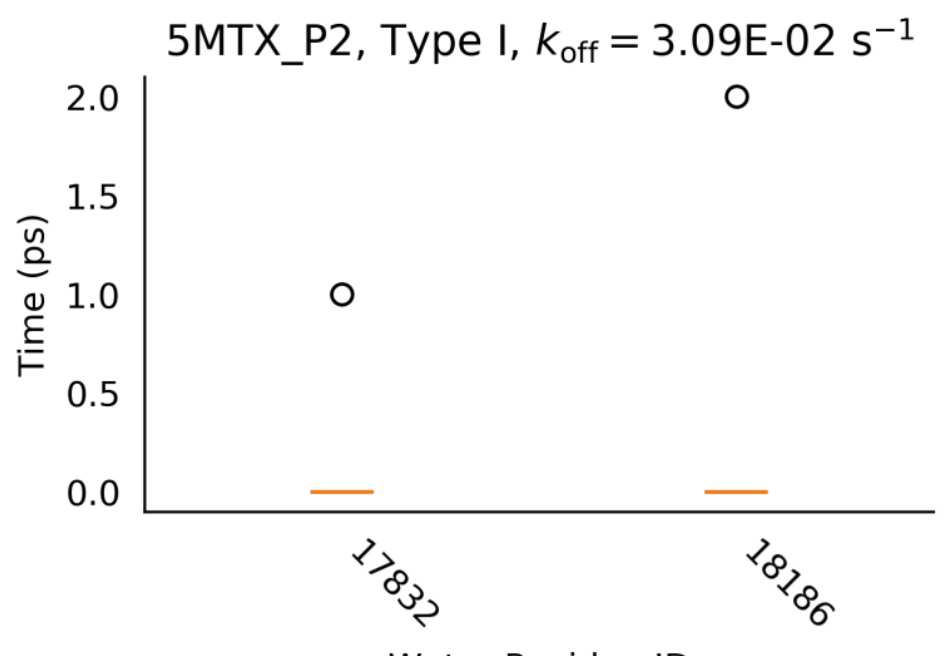

Water Residue ID

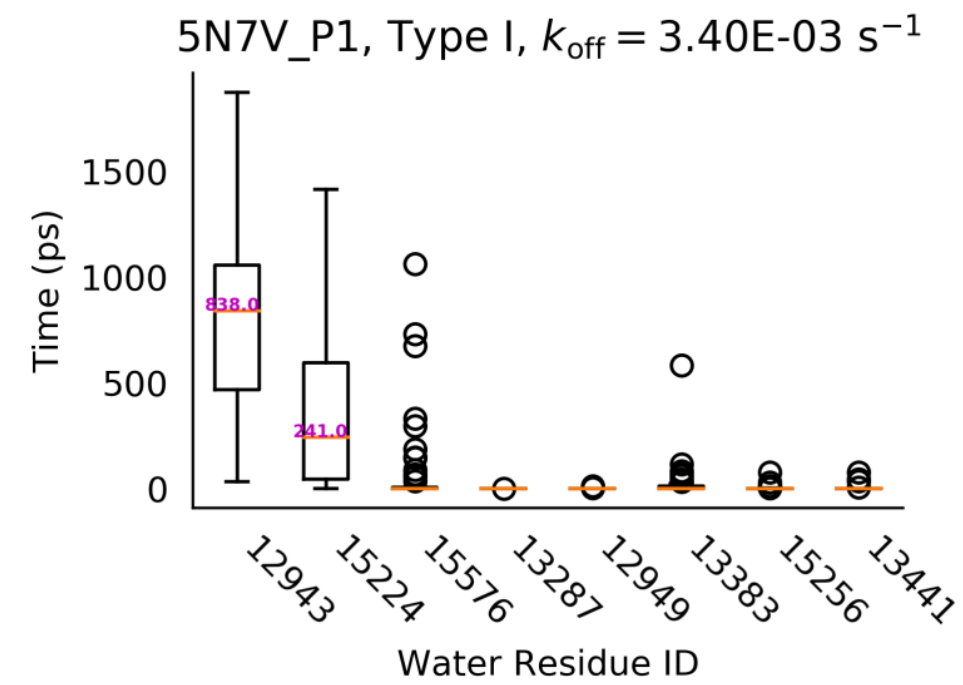



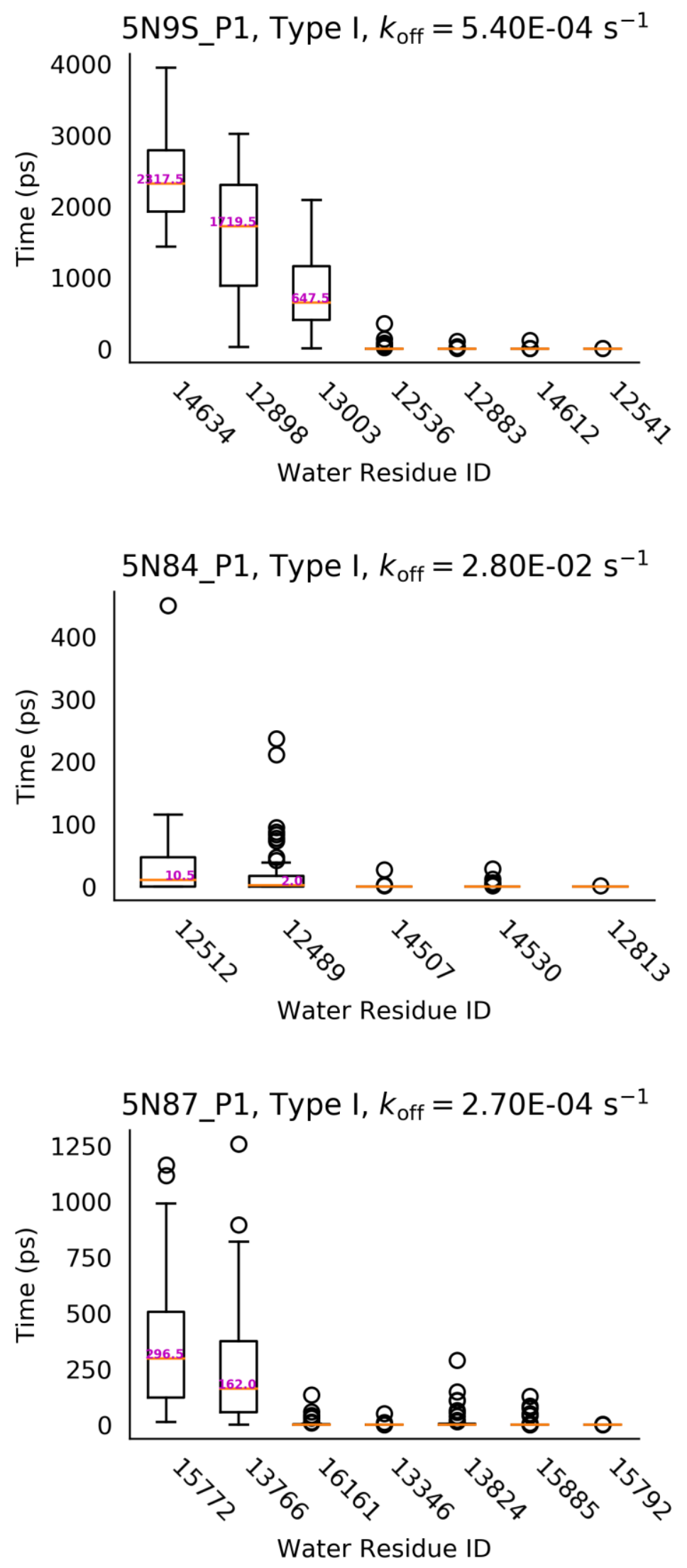

78 

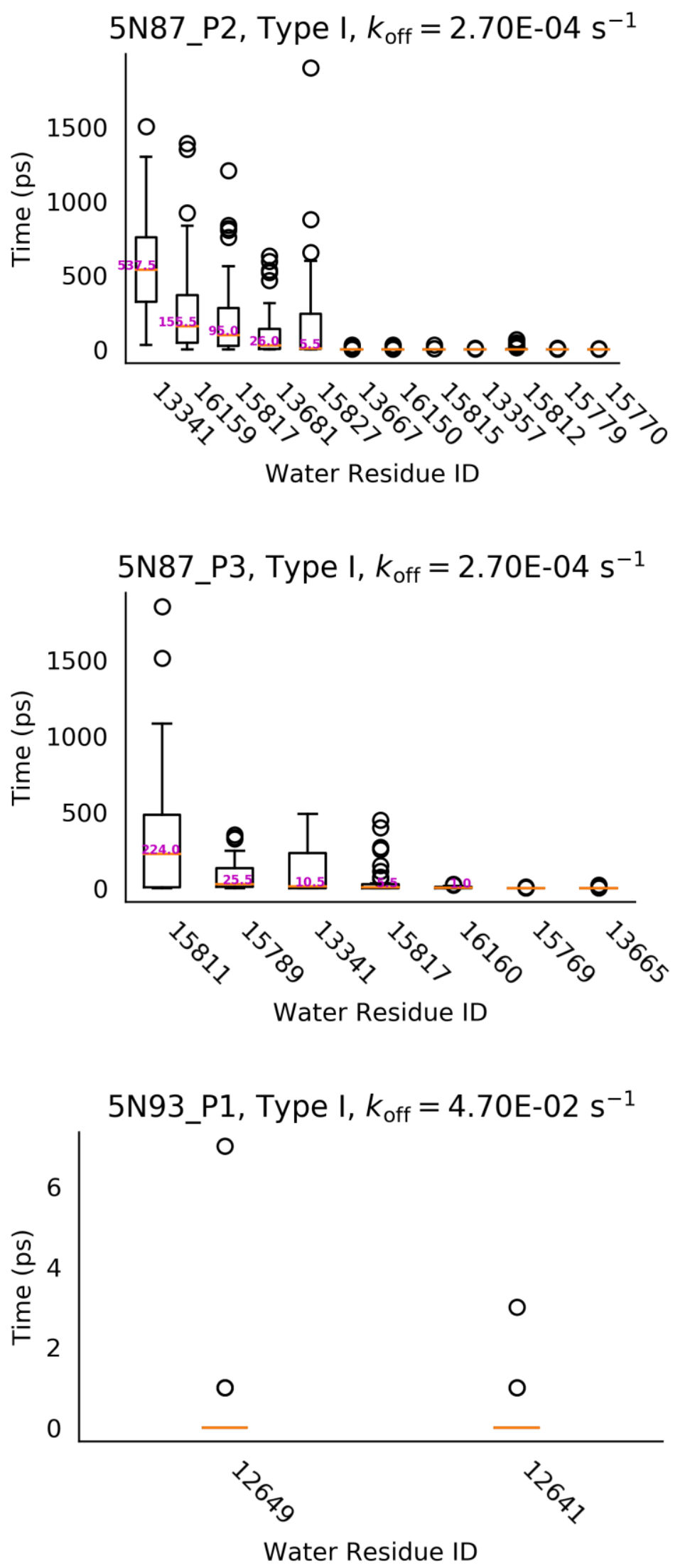

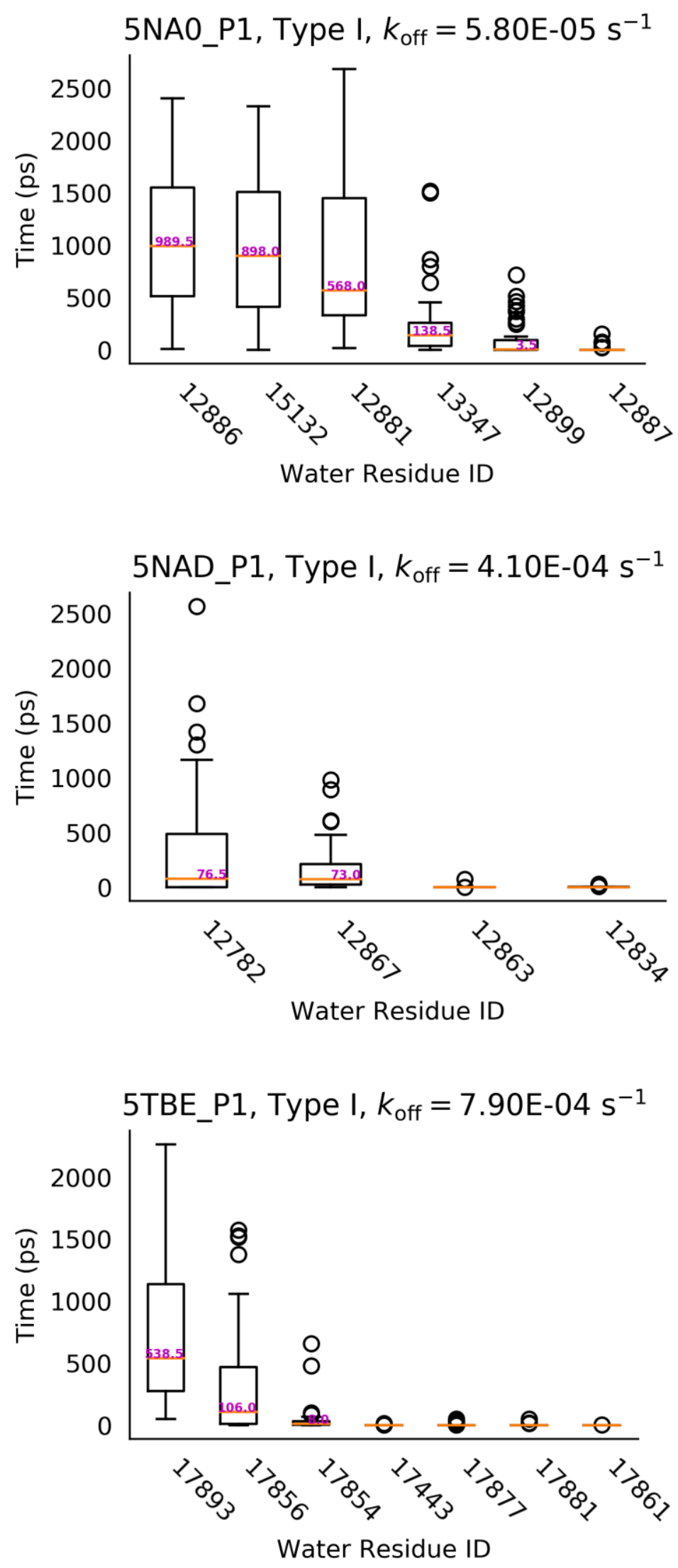

80 

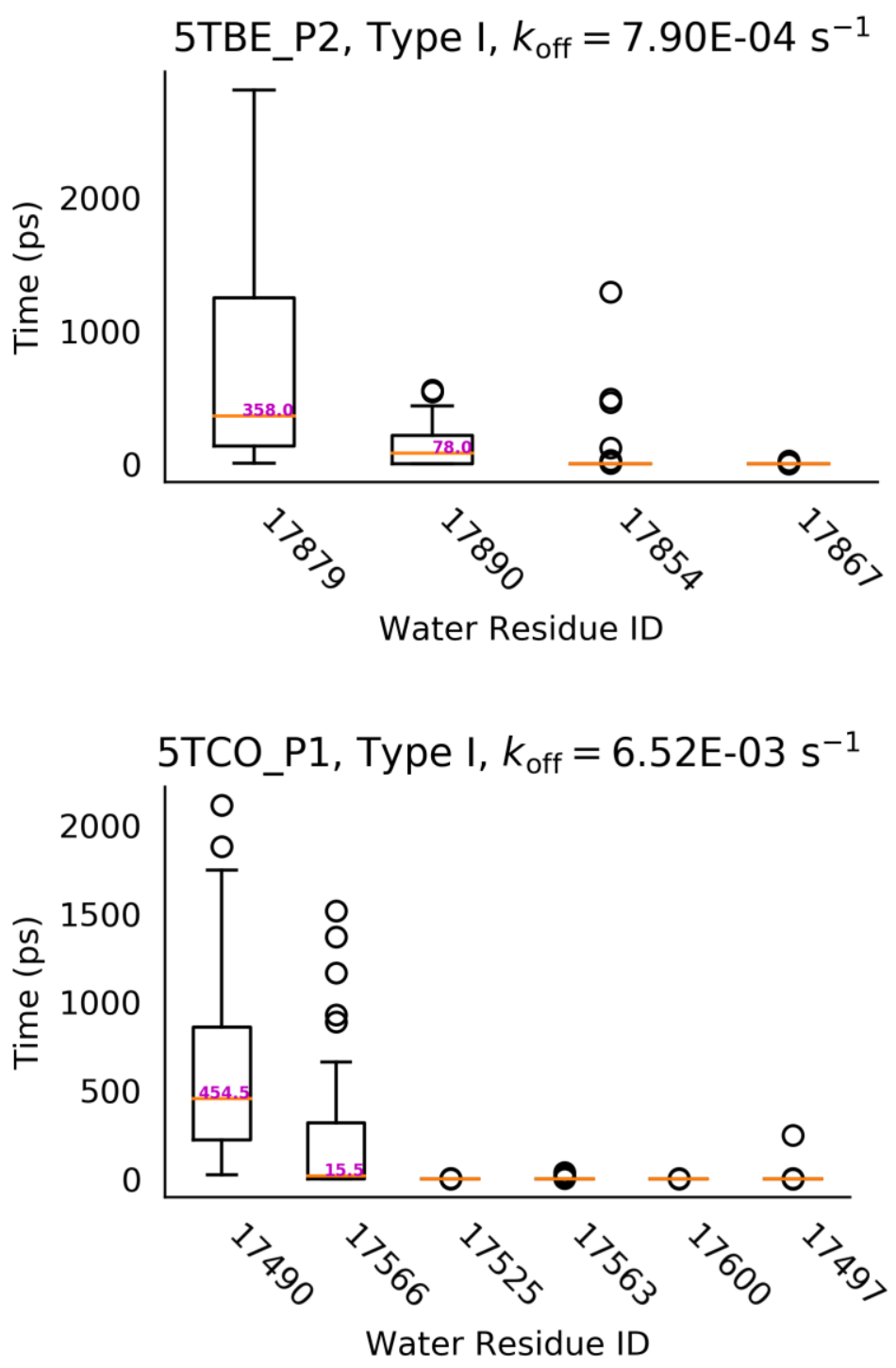
